\title{
Finite element approximation of a phase field model arising in nanostructure patterning
}

\author{
Robert Nürnberg and Edward J. W. Tucker
}

\begin{abstract}
We consider a fully practical finite element approximation of the nonlinear parabolic Cahn-Hilliard system

$$
\underline{\nabla} \cdot(c(\cdot, u) \underline{\nabla} \phi)=0 \text {, }
$$$$
\gamma \frac{\partial u}{\partial t}-\underline{\nabla} \cdot(\underline{\nabla} w)=0, \quad w=-\gamma \Delta u+\gamma^{-1} \Psi^{\prime}(u)-\frac{1}{2} \alpha c^{\prime}(\cdot, u)|\underline{\nabla} \phi|^{2},
$$

subject to an initial condition $u^{0}(.) \in[-1,1]$ on the conserved order parameter $u \in[-1,1]$, and mixed boundary conditions. Here $\gamma \in \mathbb{R}_{>0}$ is the interfacial parameter, $\alpha \in \mathbb{R}_{\geq 0}$ is the field strength parameter, $\Psi$ is the obstacle potential, $c(\cdot, u)$ is the diffusion coefficient, and $c^{\prime}(\cdot, u)$ denotes differentiation with respect to the second argument. Furthermore, $w$ is the chemical potential and $\phi$ is the electrostatic potential. The system, in the context of nanostructure patterning, has been proposed to model the manipulation of morphologies in organic solar cells with the help of an applied electric field. In the limit $\gamma \rightarrow 0$, it reduces to a sharp interface problem that models the evolution of an unstable interface between two dielectric media in the presence of a quasi-static electric field.

On introducing a finite element approximation for the above Cahn-Hilliard system, we prove existence and stability of a discrete solution. Moreover, in the case of two space dimensions, we are able to derive a convergence result. We demonstrate the practicality of our finite element approximation with several numerical simulations in two and three space dimensions.
\end{abstract}

\section{Introduction}

In today's energy dependent world, organic solar cells represent a cheap and easilymanufactured form of renewable energy. Organic cells consist of thin polymer films layered between two electrodes, and their efficiency is limited by the structure or morphology of their internal polymer layers. Here the optimal morphology is a "pillar" structure, a fingerlike arrangement of the two layers, and it has been suggested in [13] that by applying an electric field the morphology of the two layers can be affected in order to yield the desired structure. 
In particular, the authors in [13] and [21, 22] propose that the desired pillar growth morphology in solar panels can be modelled using the Cahn-Hilliard equation with an electric field. The Cahn-Hilliard equation, see [14], is used to model many physical phenomena, including spinodal decomposition, phase separation in the quenching of alloys, and the evolution of thin polymer films subjected to an electrostatic field. The Cahn-Hilliard based system considered by Kim and Lu in [21, 22] is solved using a Fourier Spectral method, with numerous numerical results presented, but no analysis of the numerical method or the underlying system of partial differential equations. Their model refers to a polymer-air arrangement for the two distinct dielectric media, but polymerpolymer arrangements are also possible, see e.g. [13, 20, 28]. For the model considered in this paper the precise type of phase arrangement do not matter, but in line with the work in $[21,22]$ we will occasionally refer to the two phases as polymer and air. We note that the authors in $[21,22]$ also consider the effect of viscous flow in the polymer layer. For simplicity, in this paper, we omit this kinetic effect in the model.

It is our goal to present a stable fully practical finite element approximation to the mentioned Cahn-Hilliard system. We will present the phase field model in detail below. In addition, a main contribution of the paper will be convergence result for our finite element approximation in two space dimensions.

Let $\Omega:=\left(-L_{1}-a, L_{1}\right) \times\left(-L_{2}, L_{2}\right)$ for $d=2$, and $\Omega:=\left(-L_{1}-a, L_{1}\right) \times\left(-L_{2}, L_{2}\right) \times$ $\left(-L_{3}, L_{3}\right)$ for $d=3$ be the domain in $\mathbb{R}^{d}$, with boundary $\partial \Omega$, and unit outward normal $\underline{\nu}_{\partial \Omega}$ to the boundary. Here $L_{i}>0, i=1 \rightarrow d$, and $a>0$ specify the dimensions of $\Omega$. Let $\partial \Omega$ denote the boundary of $\Omega$, and let $\partial_{D}^{-} \Omega:=\left\{\underline{x} \in \partial \Omega: x_{1}=-L_{1}-a\right\}$ and $\partial_{D}^{+} \Omega:=\left\{\underline{x} \in \partial \Omega: x_{1}=L_{1}\right\}$; see also Figure 1. We define $\partial_{D} \Omega:=\partial_{D}^{-} \Omega \cup \partial_{D}^{+} \Omega$, and prescribe Dirichlet boundary conditions for the electric field on $\partial_{D} \Omega$. On the remaining boundary $\partial_{N} \Omega:=\partial \Omega \backslash \overline{\partial_{D} \Omega}$ we have Neumann boundary conditions for the electric field. We split the domain $\Omega$ into film and substrate regions, by defining the substrate $\Omega_{s}:=$ $\left\{\underline{x} \in \Omega: x_{1}<-L_{1}\right\}$ to be a thin region of thickness $a$ on the left hand side of the domain, see Figure 1. The film region is defined as $\Omega_{f}:=\Omega \backslash \bar{\Omega}_{s}=\left(-L_{1}, L_{1}\right) \times \cdots \times\left(-L_{d}, L_{d}\right)$.

We introduce the interfacial parameter $\gamma \in \mathbb{R}_{>0}$, and the conserved order parameter $u(\cdot, t) \in \mathbb{R}$. Here $u$ denotes the concentration by volume fraction of polymer material: $u=1$ for the polymer, and $u=-1$ for the air. We introduce also the chemical potential $w(\cdot, t)$ and the electric field potential $\phi(\cdot, t)$. The system of equations we want to consider in this paper is then given as follows. Find functions $u(\cdot, t): \Omega_{f} \rightarrow[-1,1], w(\cdot, t): \Omega_{f} \rightarrow$ $\mathbb{R}$, and $\phi(\cdot, t): \Omega \rightarrow \mathbb{R}$ such that for almost all $t \in(0, T]$, where $T>0$ is a fixed positive time, it holds that

$$
\begin{aligned}
& \gamma \frac{\partial u}{\partial t}-\underline{\nabla} \cdot(\underline{\nabla} w)=0 \\
& w \in-\gamma \Delta u+\gamma^{-1} \partial \Psi(u)-\frac{1}{2} \alpha c^{\prime}(\underline{x})|\underline{\nabla} \phi|^{2} \\
& \underline{\nabla} w \cdot \underline{\nu}_{\partial \Omega}=\underline{\nabla} u \cdot \underline{\nu}_{\partial \Omega}=0 \\
& u(\underline{x}, 0)=u^{0}(\underline{x}) \in[-1,1] \\
& \underline{\nabla} \cdot(c(\underline{x}, u) \underline{\nabla} \phi)=0 \\
& c(\underline{x}, u) \underline{\nabla} \phi \cdot \underline{\nu}_{\partial \Omega}=0 \quad \text { on } \partial_{N} \Omega \text {, } \\
& \phi=g^{ \pm} \\
& \text {in } \Omega_{f} \text {, } \\
& \text { in } \Omega_{f} \text {, } \\
& \text { on } \partial \Omega_{f} \text {, } \\
& \forall \underline{x} \in \Omega_{f}, \\
& \text { in } \Omega \text {, } \\
& \text { on } \partial_{D}^{ \pm} \Omega \text {, }
\end{aligned}
$$




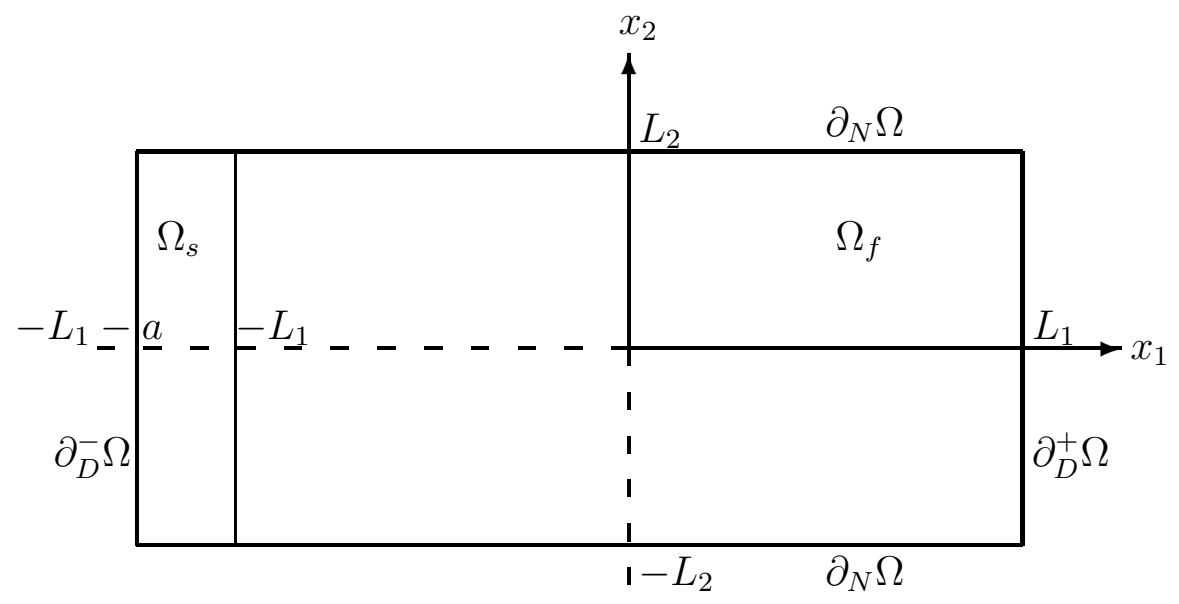

Figure 1: Diagram of the domain $\Omega$ for $d=2$, demonstrating the substrate region $\Omega_{s}$ of thickness $a$, and the film region $\Omega_{f}:=\Omega \backslash \bar{\Omega}_{s}$.

where

$$
g^{ \pm}:=x_{1}= \begin{cases}L_{1} & \text { on } \partial_{D}^{+} \Omega, \\ -L_{1}-a & \text { on } \partial_{D}^{-} \Omega .\end{cases}
$$

In $(1.1 \mathrm{a}-\mathrm{f}), \alpha \geq 0$ is a given constant relating to the strength of the electric field, and

$$
\Psi(s):= \begin{cases}\frac{1}{2}\left(1-s^{2}\right) & \text { if }|s| \leq 1 \\ \infty & \text { if }|s|>1\end{cases}
$$

with $\partial \Psi$ denoting the Fréchet subdifferential of $\Psi$; see e.g. [26]. The obstacle potential (1.3) restricts $u(\cdot, \cdot) \in[-1,1]$, and allows us to easily identify the polymer region $(u=1)$ and the non-polymer region $(u=-1)$, see $[9,10]$. Moreover, we note that the chemical potential $w$ is given as the variational derivative with respect to $u$ of the free energy

$$
J(\chi, \eta):=\int_{\Omega}\left\{\frac{1}{2} \gamma|\underline{\nabla} \chi|^{2}+\gamma^{-1} \Psi(\chi)-\frac{1}{2} \alpha c(\underline{x}, \chi)|\underline{\nabla} \eta|^{2}\right\} \mathrm{d} \underline{x} .
$$

In addition, we also define the diffusion coefficient

$$
c(\underline{x}, \chi):=\left\{\begin{array}{ll}
c_{0}+\frac{1}{2} c_{1}(1+\chi) & \underline{x} \in \overline{\Omega_{f}}, \\
c_{0}+c_{1} & \underline{x} \in \bar{\Omega} \backslash \overline{\Omega_{f}},
\end{array} \quad \forall \chi \in[-1,1],\right.
$$

where $c_{0}, c_{1} \in \mathbb{R}_{>0}$. It follows from (1.5a) that

$$
c^{\prime}(\underline{x}, \chi):=\left\{\begin{array}{ll}
\frac{1}{2} c_{1} & \underline{x} \in \overline{\Omega_{f}}, \\
0 & \underline{x} \in \bar{\Omega} \backslash \overline{\Omega_{f}},
\end{array} \quad \forall \chi \in[-1,1],\right.
$$


where $c^{\prime}(\underline{x}, \cdot)$ denotes differentiation with respect to the second variable. In view of the diffusion coefficient (1.5a), we also make the following definitions:

$$
\begin{aligned}
& c_{\max }=\max _{(\underline{x}, \chi) \in \Omega \times[-1,1]} c(\underline{x}, \chi)=c_{0}+c_{1}>0 ; \quad c_{\min }=\min _{(\underline{x}, \chi) \in \Omega \times[-1,1]} c(\underline{x}, \chi)=c_{0}>0 ; \\
& c_{\max }^{\prime}=\max _{(\underline{x}, \chi) \in \Omega \times[-1,1]} \frac{\partial c}{\partial \chi}(\underline{x}, \chi)=c_{f}^{\prime}:=\frac{1}{2} c_{1}>0 .
\end{aligned}
$$

The initial condition $u^{0} \in C(\bar{\Omega})$ is assumed to satisfy $\left|u^{0}(\underline{x})\right| \leq 1$, for all $\underline{x} \in \overline{\Omega_{f}}$.

The model $(1.1 \mathrm{a}-\mathrm{f})$ is very similar to the model $(\mathrm{P})$ in [4], which means that the numerical analysis presented in this paper will often be based on ideas from [4]. However, there are some key differences between the two models. Firstly, the quasistatic equilibrium equation for the linear elasticity in [4] is replaced by the second order elliptic problem $(1.1 \mathrm{e}, \mathrm{f})$ for the electric potential. Secondly, the electric potential term in the energy (1.4) is negative, whereas the corresponding elastic energy term in [4] is positive. These two differences, in particular, make the numerical analysis for $(1.1 \mathrm{a}-$ f) more difficult. Finally, in our model the Cahn-Hilliard mobility function is nondegenerate, whereas the paper [4] considers a degenerate diffusional mobility. Here we stress that in contrast to the models considered in $[13,21,22]$, where for simplicity $(1.1 \mathrm{a}, \mathrm{b})$ are defined over $\Omega$ with the help of a degenerate, space-dependent mobility function $b$, we restrict the definitions of $u$ and $w$ to the film region $\Omega_{f}$.

We remark that a solution $(\phi, u, w)$ to $(1.1 \mathrm{a}-\mathrm{c})$ formally satisfies the energy bound

$$
\frac{\mathrm{d}}{\mathrm{d} t} J(u, \phi)+\gamma^{-1} \int_{\Omega_{f}}|\underline{\nabla} w|^{2} \mathrm{~d} \underline{x} \leq 0 .
$$

Moreover, we note that it immediately follows from (1.1a,c) that

$$
\frac{\mathrm{d}}{\mathrm{d} t} \int_{\Omega_{f}} u \mathrm{~d} \underline{x}=0,
$$

i.e. that mass conservation holds for the system $(1.1 \mathrm{a}-\mathrm{f})$. It is the aim of this paper to introduce a finite element approximation of $(1.1 \mathrm{a}-\mathrm{f})$ that satisfies discrete analogues of (1.7) and (1.8). In the case of $d=2$, this will enable us in addition to prove convergence, and so existence of a weak solution to $(1.1 \mathrm{a}-\mathrm{f})$.

Following the procedure in [11], see also [23], it can be shown with the help of formal asymptotic expansions that the zero level sets of the solutions $u$ to $(1.1 \mathrm{a}-\mathrm{f})$, in the limit $\gamma \rightarrow 0$, converge to an interface $(\Gamma(t))_{t \in[0, T]}$ that moves according to a generalized MullinsSekerka type problem. In particular, the sharp interface motion is given by a modified Hele-Shaw model, with steady-state diffusion in $\Omega_{f}$ away from the interface $\Gamma(t)$. On the interface, a modified Gibbs-Thompson condition ensures local equilibrium with terms due to the electric field included. Full details of the formal asymptotics and the sharp interface limit can be found in [29].

The layout of this paper is as follows. In Section 2, we present our notation and some key regularity results required for stability and convergence results. In Section 2.1 we 
present the finite element spaces, and some further regularity properties required for the analysis. We then proceed in Section 3 to introduce our finite element approximation for the phase field model. Here, in Section 3.1 we present a fixed point iteration and a subsequent existence result for our scheme, while in Section 3.2 we prove a stability result. This enables us to show convergence, in the case of two space dimensions, in Section 4. Finally, in Section 5 we give some details on the employed solution methods and we present numerical results in two and three dimensions.

\section{Notation and auxiliary results}

For $D \subset \mathbb{R}^{d}, d=2$ or 3 , we adopt the standard notation for Sobolev spaces, denoting the norm of $W^{m, q}(D),\left(m \in \mathbb{N} \cup\left\{\frac{1}{2}\right\}, q \in[1, \infty]\right)$, by $\|\cdot\|_{m, q, D}$ and the seminorm by $|\cdot|_{m, q, D}$. For $q=2, W^{m, 2}(D)$ will be denoted by $H^{m}(D)$ with the associated norm and seminorm written as $\|\cdot\|_{m, D}$ and $|\cdot|_{m, D}$, respectively. We also choose to drop the domain subscript on the above norms and seminorms in the case $D \equiv \Omega$. Throughout, $(\cdot, \cdot)$ denotes the standard $L^{2}$ inner product over $\Omega$.

We define

$$
f_{D} \eta \mathrm{d} \underline{x}:=\frac{1}{|D|} \int_{D} \eta \mathrm{d} \underline{x} \quad \forall \eta \in L^{1}(D),
$$

where $|D|$ is the measure of a domain $D$. For later purposes, we recall the following well-known Sobolev interpolation result, e.g., see [1]: Let $q \in[1, \infty], r \in[q, \infty]$ if $q>d$, $r \in[q, \infty)$ if $q=d$, and $r \in\left[q, \frac{d q}{d-q}\right]$ if $q \in[1, d)$; and $\mu:=\frac{d}{q}-\frac{d}{r}$. Then the following inequality holds:

$$
|z|_{0, r} \leq C|z|_{0, q}^{1-\mu}\|z\|_{1, q}^{\mu} \quad z \in W^{1, q}(\Omega),
$$

where $C$ depends only on $\Omega, q$ and $r$. For future reference, we make note of the generalised Young's inequality

$$
r s \leq \frac{1}{p}(\varsigma r)^{p}+\frac{1}{q}\left(\varsigma^{-1} s\right)^{q} \quad \forall r, s \in \mathbb{R}, \varsigma \in \mathbb{R}_{>0}, p \in(1, \infty) \text { with } \frac{1}{p}+\frac{1}{q}=1 .
$$

In addition we make note of the elementary inequality

$$
|r+s|^{p} \leq 2^{p-1}\left(|r|^{p}+|s|^{p}\right) \quad \forall r, s \in \mathbb{R}, p \in[1, \infty) \text {. }
$$

For later purposes, we recall the following compactness results. Let $X, Y$, and $Z$ be Banach spaces with a compact embedding $X \hookrightarrow Y$ and a continuous embedding $Y \hookrightarrow Z$. Then the embeddings

$$
\left\{\eta \in L^{2}(0, T ; X): \frac{\partial \eta}{\partial t} \in L^{2}(0, T ; Z)\right\} \hookrightarrow L^{2}(0, T ; Y)
$$

and

$$
\left\{\eta \in L^{\infty}(0, T ; X): \frac{\partial \eta}{\partial t} \in L^{2}(0, T ; Z)\right\} \hookrightarrow C([0, T] ; Y)
$$


are compact.

Let $(\cdot, \cdot)_{\Omega_{f}}$ denote the $L^{2}$ inner product over $\Omega_{f}$. It is convenient to introduce the "inverse Laplacian" operator $\mathcal{G}_{f}: Y_{f} \rightarrow Z_{f}$ such that

$$
\left(\underline{\nabla}\left[\mathcal{G}_{f} z\right], \underline{\nabla} \eta\right)_{\Omega_{f}}=\langle z, \eta\rangle_{H^{1}\left(\Omega_{f}\right)} \quad \forall \eta \in H^{1}\left(\Omega_{f}\right)
$$

where $Y_{f}:=\left\{z \in\left(H^{1}\left(\Omega_{f}\right)\right)^{\prime}:\langle z, 1\rangle_{H^{1}\left(\Omega_{f}\right)}=0\right\}, Z_{f}:=\left\{z \in H^{1}\left(\Omega_{f}\right):(z, 1)_{\Omega_{f}}=0\right\}$, and $\langle\cdot, \cdot\rangle_{H^{1}\left(\Omega_{f}\right)}$ denotes the duality pairing between $\left(H^{1}\left(\Omega_{f}\right)\right)^{\prime}$ and $H^{1}\left(\Omega_{f}\right)$. The well-posedness of $\mathcal{G}_{f}$ follows from the Lax-Milgram theorem and the Poincaré inequality.

For $p \in(1, \infty)$, we introduce also the spaces

$$
S_{0}^{p}:=\left\{\eta \in W^{1, p}(\Omega): \eta=0 \text { on } \partial_{D} \Omega\right\},
$$

and set $S_{0}:=S_{0}^{2}$. The following lemma holds:

Lemma 2.1. There exists a $\delta>0$ such that for all $p \in\left[\frac{2+\delta}{1+\delta}, 2+\delta\right]$ there is a $\beta(p) \geq 1$ satisfying

$$
|\underline{\nabla} z|_{0, p} \leq \beta(p) \sup _{0 \neq \eta \in S_{0}^{q}} \frac{(\underline{\nabla} z, \underline{\nabla} \eta)}{|\underline{\nabla} \eta|_{0, q}} \quad \forall z \in S_{0}^{p},
$$

where $\frac{1}{p}+\frac{1}{q}=1$. Moreover, $\beta$ is continuous on the interval $\left[\frac{2+\delta}{1+\delta}, 2+\delta\right]$ and $\beta(p) \rightarrow$ $\beta(2)=1$ as $p \rightarrow 2$.

Proof The proof is similar to the proof of [4, Lemma 1.1]. For $z \in S_{0}^{p}$, we define

$$
\mathcal{S}(\underline{F}):=(\underline{\nabla} z, \underline{F}) \quad \forall \underline{F} \in M:=\left\{\underline{\nabla} \eta: \eta \in S_{0}^{q}\right\} .
$$

We note that $\mathcal{S}$ is a continuous linear functional on the closed subspace $M$ of $\left[L^{q}(\Omega)\right]^{d}$, with norm

$$
\|\mathcal{S}\|=\sup _{0 \neq \underline{F} \in M} \frac{\mathcal{S}(\underline{F})}{|\underline{F}|_{0, q}}=\sup _{0 \neq \eta \in S_{0}^{q}} \frac{(\underline{\nabla} z, \underline{\nabla} \eta)}{|\underline{\nabla} \eta|_{0, q}} .
$$

The Hahn-Banach theorem and the fact that $\left(\left[L^{q}(\Omega)\right]^{d}\right)^{\prime} \cong\left[L^{p}(\Omega)\right]^{d}$ imply the existence of a $\underline{G}_{z} \in\left[L^{p}(\Omega)\right]^{d}$ such that

$$
(\underline{\nabla} z, \underline{F})=\left(\underline{G}_{z}, \underline{F}\right) \quad \forall \underline{F} \in M \quad \text { and } \quad\left|\underline{G}_{z}\right|_{0, p}=\sup _{0 \neq \eta \in S_{0}^{q}} \frac{(\underline{\nabla} z, \underline{\nabla} \eta)}{|\underline{\nabla} \eta|_{0, q}} .
$$

We also introduce the linear operator $Q:\left[L^{p}(\Omega)\right]^{d} \rightarrow\left[L^{p}(\Omega)\right]^{d}$, such that $Q \underline{F}=\underline{\nabla} f_{F}$, where $f_{F} \in S_{0}^{p}$ is such that

$$
\left(\underline{\nabla} f_{F}, \underline{\nabla} \eta\right)=(\underline{F}, \underline{\nabla} \eta) \quad \forall \eta \in S_{0}^{q}
$$

We know that $Q$ is well-posed for $p=2$, by the Lax-Milgram theorem, and that $\|Q\|_{2}=1$.

Let $\delta>0$ be defined as in Lemma A.4 (see the appendix). Then it holds that

$$
\begin{aligned}
|Q \underline{F}|_{0, p} & \leq C(p)\left[|Q \underline{F}|_{0,2}+|\underline{F}|_{0, p}\right] \leq C(p)\left[\|Q\|_{2}|\underline{F}|_{0,2}+|\underline{F}|_{0, p}\right] \\
& \leq C(p)\left[|\underline{F}|_{0,2}+|\underline{F}|_{0, p}\right] \leq C(p)|\underline{F}|_{0, p} \quad \forall p \in[2,2+\delta] .
\end{aligned}
$$


The above shows that $Q$ is a bounded linear operator for $p \in[2,2+\delta]$ and that $\|Q\|_{p} \leq$ $C(p)$.

We now want to show that $Q$ is also a continuous linear operator on $\left[L^{q}(\Omega)\right]^{d}$, where $q$ is such that $\frac{1}{p}+\frac{1}{q}=1$ for a $p \in[2,2+\delta]$. To do so we approximate $\underline{F} \in\left[L^{q}(\Omega)\right]^{d}$ by $\underline{F}_{k} \in\left[L^{2}(\Omega)\right]^{d}$ such that $\left|\underline{F}-\underline{F}_{k}\right|_{0, q} \rightarrow 0$ as $k \rightarrow \infty$. We have that $Q \underline{F}_{k}=\underline{\nabla} f_{F_{k}}$, where $f_{F_{k}} \in S_{0}$ is such that

$$
\left(\underline{\nabla} f_{F_{k}}, \underline{\nabla} \eta\right)=\left(\underline{F}_{k}, \underline{\nabla} \eta\right) \quad \forall \eta \in S_{0} .
$$

Given $\underline{H} \in\left[L^{p}(\Omega)\right]^{d}$, we have that $Q \underline{H}=\underline{\nabla} f_{H}$, where $f_{H} \in S_{0}^{p}$ is such that

$$
\left(\underline{\nabla} f_{H}, \underline{\nabla} \eta\right)=(\underline{H}, \underline{\nabla} \eta) \quad \forall \eta \in S_{0}^{q} .
$$

Applying (2.10), (2.11), and noting that $\left[L^{p}(\Omega)\right]^{d} \subset\left[L^{2}(\Omega)\right]^{d} \subset\left[L^{q}(\Omega)\right]^{d}$, we obtain that

$$
\left(Q \underline{F}_{k}, \underline{H}\right)=\left(\underline{\nabla} f_{F_{k}}, \underline{H}\right)=\left(\underline{\nabla} f_{F_{k}}, \underline{\nabla} f_{H}\right)=\left(\underline{F}_{k}, \underline{\nabla} f_{H}\right)=\left(\underline{F}_{k}, Q \underline{H}\right) \quad \forall \underline{H} \in\left[L^{p}(\Omega)\right]^{d} .
$$

Hence we obtain from (2.12) and (2.9) that

$$
\left|\left(Q \underline{F}_{k}, \underline{H}\right)\right|=\left|\left(\underline{F}_{k}, Q \underline{H}\right)\right| \leq|Q \underline{H}|_{0, p}\left|\underline{F}_{k}\right|_{0, q} \leq\|Q\|_{p}|\underline{H}|_{0, p}\left|\underline{F}_{k}\right|_{0, q} \quad \forall \underline{H} \in\left[L^{p}(\Omega)\right]^{d} .
$$

Therefore we have that

$$
\left|Q \underline{F}_{k}\right|_{0, q} \leq\|Q\|_{p}\left|\underline{F}_{k}\right|_{0, q} .
$$

Combining a Friedrich's inequality, (2.13), and $\underline{F}_{k} \rightarrow \underline{F}$ in $\left[L^{q}(\Omega)\right]^{d}$ as $k \rightarrow \infty$, we have that

$$
\left\|f_{F_{k}}\right\|_{1, q} \leq C\left|\underline{\nabla} f_{F_{k}}\right|_{0, q} \leq C\|Q\|_{p}\left|\underline{F}_{k}\right|_{0, q} \leq C \quad \forall k \in \mathbb{N} .
$$

Therefore we can choose a subsequence $\left\{f_{F_{k_{i}}}\right\}_{i \geq 0}$ such that

$$
f_{F_{k_{i}}} \rightarrow f_{F} \quad \text { weakly in } S_{0}^{q}, \quad \text { as } i \rightarrow \infty \text {. }
$$

Combining (2.14) and (2.10) for $\eta \in S_{0}^{p}$, yields that

$$
\left(\underline{F}_{k_{i}}, \underline{\nabla} \eta\right)=\left(\underline{\nabla} f_{F_{k_{i}}}, \underline{\nabla} \eta\right) \rightarrow\left(\underline{\nabla} f_{F}, \underline{\nabla} \eta\right) \quad \forall \eta \in S_{0}^{p},
$$

as $i \rightarrow \infty$. The strong convergence of $\left\{\underline{F}_{k}\right\}$ then yields that

$$
\left(\underline{\nabla} f_{F}, \underline{\nabla} \eta\right)=(\underline{F}, \underline{\nabla} \eta) \quad \forall \eta \in S_{0}^{p} .
$$

The fundamental lemma of the calculus of variations, see e.g. [2, Lemma 2.21], together with a Friedrich's inequality, then yields that $f_{F}$ is unique, and therefore the whole sequence converges, i.e. $f_{F_{k}} \rightarrow f_{F}$ weakly in $S_{0}^{q}$.

Hence, on setting $Q \underline{F}:=\underline{\nabla} f_{F}$, we have that

$$
Q \underline{F}_{k} \rightarrow Q \underline{F} \quad \text { weakly in }\left[L^{q}(\Omega)\right]^{d} .
$$

Therefore from $(2.12)$ we have that $(Q \underline{F}, \underline{H})=(\underline{F}, Q \underline{H})$ for all $\underline{H} \in\left[L^{p}(\Omega)\right]^{d}$, and similarly to (2.13) it follows that

$$
|Q \underline{F}|_{0, q} \leq\|Q\|_{p}|\underline{F}|_{0, q},
$$


and so $\|Q\|_{q} \leq\|Q\|_{p}$. Moreover, the above has established that, given $\underline{H} \in\left[L^{p}(\Omega)\right]^{d}$

$$
(Q \underline{F}, \underline{H})=(\underline{F}, Q \underline{H}) \quad \forall \underline{F} \in\left[L^{q}(\Omega)\right]^{d},
$$

and so also $\|Q\|_{p} \leq\|Q\|_{q}$. Hence $\|Q\|_{q}=\|Q\|_{p}$.

The Riesz-Thorin theorem, see [8], then implies that $\|Q\|_{p} \leq\|Q\|_{s}^{1-\varsigma}\|Q\|_{r}^{\varsigma}$ for all $\frac{2+\delta}{1+\delta} \leq s \leq p \leq r \leq 2+\delta$ such that $\frac{1}{p}=(1-\varsigma) \frac{1}{s}+\varsigma \frac{1}{r}$ and $\varsigma \in[0,1]$. It follows that $\log \|Q\|_{p} \leq(1-\varsigma) \log \|Q\|_{s}+\varsigma \log \|Q\|_{r}$, that is, $\log \|Q\|_{p}$ is a convex function of $\frac{1}{p}$, and therefore $\log \|Q\|_{p}$ is a continuous function of $p$ with $\|Q\|_{2}=1$, since convex functions are continuous.

Therefore we have that $\|Q\|_{p}=\beta(p)$ for $\beta \in C\left[\frac{2+\delta}{1+\delta}, 2+\delta\right]$, and $\|Q\|_{2}=1$ implies that $\beta(2)=1$. Finally it follows from (2.8) and $z \in S_{0}^{p}$ that $\underline{\nabla} z=Q \underline{G}_{z}$ and hence

$$
|\underline{\nabla} z|_{0, p} \leq\|Q\|_{p}\left|\underline{G}_{z}\right|_{0, p}=\beta(p)\left|\underline{G}_{z}\right|_{0, p} .
$$

Therefore the desired result (2.7) follows from (2.15) and (2.8).

\subsection{Finite element spaces}

We consider finite element approximations of $(1.1 \mathrm{a}-\mathrm{f})$ under the following assumptions on the mesh:

$\left(\mathcal{A}_{1}\right)$ For $d=2$ or 3 , let $\left\{\mathcal{T}^{h}\right\}_{h>0}$ be a family of regular partitionings of $\Omega$ into disjoint open simplices $\sigma$ with $h_{\sigma}:=\operatorname{diam}(\sigma)$ and $h:=\max _{\sigma \in \mathcal{T}^{h}} h_{\sigma}$ so that $\bar{\Omega}=\bigcup_{\sigma \in \mathcal{T}^{h}} \bar{\sigma}, \bar{\Omega}_{s}=$ $\bigcup_{\sigma \in \mathcal{T}_{s}^{h}} \bar{\sigma}$ and $\overline{\Omega_{f}}=\bigcup_{\sigma \in \mathcal{T}_{f}^{h}} \bar{\sigma}$, where $\mathcal{T}_{s}^{h}, \mathcal{T}_{f}^{h} \subset \mathcal{T}^{h}$. The regularity assumption implies that

$$
\frac{h_{\sigma}}{\rho_{\sigma}} \leq C \quad \forall \sigma \in \mathcal{T}^{h} \quad \forall h>0,
$$

where $C$ is independent of $h$, and $\rho_{\sigma}$ is defined to be the diameter of the largest inscribed ball of $\sigma$. In addition, it is assumed that all simplices $\sigma \in \mathcal{T}^{h}$ are non-obtuse, that is, the angle between any 2 sides $(d=2)$ or faces $(d=3)$ does not exceed $\frac{\pi}{2}$.

Associated with $\mathcal{T}^{h}$ is the finite element space

$$
S^{h}:=\left\{\chi \in C(\bar{\Omega}):\left.\chi\right|_{\sigma} \text { is linear } \forall \sigma \in \mathcal{T}^{h}\right\} \subset H^{1}(\Omega),
$$

and the following finite element spaces, which take into account the Dirichlet boundary conditions,

$$
S_{0}^{h}=\left\{\chi \in S^{h}: \chi=0 \text { on } \partial_{D} \Omega\right\} \subset S_{0}, \quad S_{g}^{h}=\left\{\chi \in S^{h}: \chi=g^{ \pm} \text {on } \partial_{D}^{ \pm} \Omega\right\},
$$

where $g^{ \pm}$is as in (1.2). In addition we introduce the spaces $S_{f}^{h}:=\left\{\chi \in C\left(\overline{\Omega_{f}}\right)\right.$ : $\left.\chi\right|_{\sigma}$ is linear $\left.\forall \sigma \in \mathcal{T}_{f}^{h}\right\} \subset H^{1}\left(\Omega_{f}\right)$ and $K_{f}^{h}:=\left\{\chi \in S_{f}^{h}:|\chi| \leq 1\right.$ in $\left.\Omega_{f}\right\} \subset K_{f}:=$ $\left\{\eta \in H^{1}\left(\Omega_{f}\right):|\eta| \leq 1\right.$ a.e. in $\left.\Omega_{f}\right\}$. 
We let $J$ be the set of nodes of $\mathcal{T}^{h}$ and $\left\{\underline{p}_{j}\right\}_{j \in J}$ the coordinates of these nodes. We let $\left\{\chi_{j}\right\}_{j \in J}$ be the standard basis functions for $S^{h}$; that is, $\chi_{j} \in S^{h}$ and $\chi_{j}\left(\underline{p}_{i}\right)=\delta_{i j}$ for all $i, j \in J$. The non-obtuse assumption yields that

$$
\int_{\sigma} \underline{\nabla} \chi_{i} \cdot \underline{\nabla} \chi_{j} \mathrm{~d} \underline{x} \leq 0 \quad i \neq j, \quad \forall \sigma \in \mathcal{T}^{h} .
$$

We introduce also the interpolation operator $\pi^{h}: C(\bar{\Omega}) \rightarrow S^{h}$, such that

$$
\left(\pi^{h} \eta\right)\left(\underline{p}_{j}\right)=\eta\left(\underline{p}_{j}\right) \quad \forall j \in J .
$$

A discrete semi-inner product on $C(\bar{\Omega})$ is then defined by

$$
\left(\eta_{1}, \eta_{2}\right)^{h}:=\int_{\Omega} \pi^{h}\left(\eta_{1}(\underline{x}) \eta_{2}(\underline{x})\right) \mathrm{d} \underline{x}=\sum_{j \in J} m_{j} \eta_{1}\left(\underline{p}_{j}\right) \eta_{2}\left(\underline{p}_{j}\right),
$$

where $m_{j}:=\left(1, \chi_{j}\right)>0$. The induced discrete seminorm is then

$$
|\eta|_{h}:=\left[(\eta, \eta)^{h}\right]^{\frac{1}{2}}=\left(\int_{\Omega} \pi^{h}\left[\eta^{2}\right] \mathrm{d} \underline{x}\right)^{\frac{1}{2}} \quad \forall \eta \in C(\bar{\Omega}) .
$$

The following are well-known results concerning the finite element space $S^{h}$ for $d=$ 2 or 3 and for any $\sigma \in \mathcal{T}^{h}, \chi, z^{h} \in S^{h}, m \in\{0,1\}, q \in[2, \infty)$ and $r \in(d, \infty]$ :

$$
\begin{array}{lr}
|\chi|_{1, \sigma} \leq C h_{\sigma}^{-1}|\chi|_{0, \sigma} ; & \forall \eta \in H^{2}(\Omega) ; \\
\left|\left(I-\pi^{h}\right) \eta\right|_{m, q} \leq C h^{2-m-d\left(\frac{1}{2}-\frac{1}{q}\right)}|\eta|_{2} & \forall \eta \in W^{1, r}(\Omega) ; \\
\left|\left(I-\pi^{h}\right) \eta\right|_{m, r} \leq C h^{1-m}|\eta|_{1, r} & \forall \eta \in W^{1, r}(\Omega) ; \\
\lim _{h \rightarrow 0}\left\|\left(I-\pi^{h}\right) \eta\right\|_{1, r} \rightarrow 0 & \\
\int_{\sigma} \chi^{2} \mathrm{~d} \underline{x} \leq \int_{\sigma} \pi^{h}\left[\chi^{2}\right] \mathrm{d} \underline{x} \leq(d+2) \int_{\sigma} \chi^{2} \mathrm{~d} \underline{x} ; & \\
\left|\left(\chi, z^{h}\right)-\left(\chi, z^{h}\right)^{h}\right| \leq\left|\left(I-\pi^{h}\right)\left(\chi z^{h}\right)\right|_{0,1} \leq C h^{1+m}|\chi|_{m}\left|z^{h}\right|_{1} . &
\end{array}
$$

Analogously on $\Omega_{f}$, we define a discrete semi-inner product on $C\left(\overline{\Omega_{f}}\right)$ by

$$
\left(\eta_{1}, \eta_{2}\right)_{\Omega_{f}}^{h}:=\int_{\Omega_{f}} \pi_{f}^{h}\left(\eta_{1}(\underline{x}) \eta_{2}(\underline{x})\right) \mathrm{d} \underline{x}=\sum_{j \in J_{f}} m_{j} \eta_{1}\left(\underline{p}_{j}\right) \eta_{2}\left(\underline{p}_{j}\right),
$$

where $\pi_{f}^{h}: C\left(\overline{\Omega_{f}}\right) \rightarrow S_{f}^{h}$ is the analogue of $\pi^{h}$, and $J_{f} \subset J$ is the set of nodes of $\mathcal{T}_{f}^{h}$. We note that the analogues of $(2.18 \mathrm{~b}-\mathrm{g})$ for $\pi^{h}$ hold for $\pi_{f}^{h}$. The induced discrete seminorm is then

$$
|\eta|_{h, \Omega_{f}}:=\left[(\eta, \eta)_{\Omega_{f}}^{h}\right]^{\frac{1}{2}}=\left(\int_{\Omega_{f}} \pi_{f}^{h}\left[\eta^{2}\right] \mathrm{d} \underline{x}\right)^{\frac{1}{2}} \quad \forall \eta \in C\left(\overline{\Omega_{f}}\right) .
$$


We introduce the mass lumped projection $Q_{f}^{h}: L^{2}\left(\Omega_{f}\right) \rightarrow S_{f}^{h}$ such that

$$
\left(Q_{f}^{h} \eta, \chi\right)_{\Omega_{f}}^{h}=(\eta, \chi)_{\Omega_{f}} \quad \forall \chi \in S_{f}^{h}
$$

and note that the projection $Q_{f}^{h}$ satisfies the analogue of $(2.20)$ if we have a quasi-uniform family of partitionings.

For certain results we require a further assumption that the mesh is quasi-uniform:

$\left(\mathcal{A}_{2}\right)$ Let $\left\{\mathcal{T}^{h}\right\}_{h>0}$ satisfy $\left(\mathcal{A}_{1}\right)$ and in addition assume that the family of partitions is quasi-uniform, that is,

$$
|\sigma| \geq C h^{d} \quad \forall \sigma \in \mathcal{T}^{h}
$$

If we have a quasi-uniform family of partitionings, it holds that

$$
\left|\left(I-Q_{f}^{h}\right) \eta\right|_{m} \leq C h^{1-m}|\eta|_{1} \quad \forall \eta \in H^{1}\left(\Omega_{f}\right), \quad m=0 \text { or } 1 .
$$

We introduce the projection operator $P^{h}: S_{0}^{1} \rightarrow S_{0}^{h}$, recall (2.6), such that

$$
\left(\underline{\nabla}\left(z-P^{h} z\right), \underline{\nabla} \chi\right)=0 \quad \forall \chi \in S_{0}^{h} .
$$

It is crucial for our analysis to prove the following result.

Lemma 2.2. Let $d=2$ and the assumptions $\left(\mathcal{A}_{1}\right)$ and $\left(\mathcal{A}_{2}\right)$ hold. Let $\delta>0$ be as defined in Lemma 2.1. Then there exists $h_{0}>0$ and $a \widehat{\beta} \in C\left(\left[\frac{2+\delta}{1+\delta}, \infty\right)\right)$ such that for all $p \in\left[\frac{2+\delta}{1+\delta}, \infty\right)$ and for all $h \in\left(0, h_{0}\right)$,

$$
\left|\underline{\nabla}\left(P^{h} z\right)\right|_{0, p} \leq \widehat{\beta}(p)|\underline{\nabla} z|_{0, p} \quad \forall z \in S_{0}^{p},
$$

with $\widehat{\beta}(p) \geq 1$ and $\hat{\beta}(p) \rightarrow \hat{\beta}(2)=1$ as $p \rightarrow 2$.

Proof The proof is very similar to that given in [4, Lemma 2.3], which was itself adapted from the proof for the Laplacian with homogeneous Dirichlet boundary conditions given in [12, Chapter 8], and based on the approach in [24]. As the proof is long, we break it up into three parts, similarly to [12, Chapter 8].

1. Reduction of (2.22) to the weighted error estimate (2.30). Given $\mathcal{T}^{h}$ and any $\underline{y} \in \Omega$, let $\sigma_{\underline{y}} \in \mathcal{T}^{h}$ be such that $\underline{y} \in \sigma_{\underline{y}}$. We then introduce $\delta_{\underline{y}}^{h} \in C_{0}^{\infty}(\bar{\Omega})$ with $\operatorname{supp}\left(\delta_{\underline{y}}^{h}\right) \subset \sigma_{\underline{y}}$ such that

$$
\int_{\sigma_{\underline{y}}} \delta_{\underline{y}}^{h} \mathrm{~d} \underline{x}=1 \quad \text { and } \quad\left\|\delta_{\underline{y}}^{h}\right\|_{m, \infty, \sigma_{\underline{y}}} \leq C h^{-(2+m)} \quad \forall m \in \mathbb{N},
$$

where we recall the assumption $\left(\mathcal{A}_{2}\right)$. For $i \in\{1,2\}$, let $f_{\underline{y}, i} \in S_{0}$ be such that

$$
\left(\underline{\nabla} f_{\underline{y}, i}, \underline{\nabla} \eta\right)=\left(\delta_{\underline{y}}^{h},[\underline{\nabla} \eta]_{i}\right)=-\left(\frac{\partial}{\partial x_{i}} \delta_{\underline{y}}^{h}, \eta\right) \quad \forall \eta \in S_{0} .
$$


It follows from the Lax-Milgram theorem and a Friedrich's inequality that (2.24) is wellposed. Moreover, $f_{\underline{y}, i} \in H^{2}(\Omega)$ from Lemma A.1 (see the appendix). We have from (2.23), (2.21) and (2.24) for all $\underline{y} \in \Omega$ and for $i \in\{1,2\}$ that

$$
\begin{aligned}
{\left[\underline{\nabla}\left(P^{h} z\right)\right]_{i}(\underline{y}) } & =\left(\delta_{\underline{y}}^{h},\left[\underline{\nabla}\left(P^{h} z\right)\right]_{i}\right)=\left(\underline{\nabla} f_{\underline{y}, i}, \underline{\nabla}\left(P^{h} z\right)\right)=\left(\underline{\nabla}\left(P^{h} f_{\underline{y}, i}\right), \underline{\nabla} z\right) \\
& =\left(\delta_{\underline{y}}^{h},[\underline{\nabla} z]_{i}\right)+\left(\underline{\nabla}\left(\left[P^{h}-I\right] f_{\underline{y}, i}\right), \underline{\nabla} z\right) \quad \forall z \in S_{0} .
\end{aligned}
$$

For any $\underline{y} \in \Omega$ and any constant $\varrho \geq 1$, we introduce the weight function

$$
\omega_{\underline{y}, \varrho}(\underline{x}):=\left(|\underline{x}-\underline{y}|^{2}+\varrho^{2} h^{2}\right)^{\frac{1}{2}} .
$$

It holds that

$$
\int_{\Omega} \omega_{\underline{y}, \rho}^{-\varsigma} \mathrm{d} \underline{x} \leq C(\varsigma)(\varrho h)^{-\varsigma+2} \quad \forall \varsigma \in(2, \infty) .
$$

Moreover, it is easily verified for any $\varsigma \in \mathbb{R}$ that

$$
\max _{\sigma \in \mathcal{T}^{h}}\left(\sup _{\underline{x} \in \sigma}\left[\omega_{\underline{y}, \varrho}(\underline{x})\right]^{\varsigma} / \inf _{\underline{x} \in \sigma}\left[\omega_{\underline{y}, \varrho}(\underline{x})\right]^{\varsigma}\right) \leq C, \quad\left|\omega_{\underline{y}, \varrho}^{\varsigma}\right|_{0, \infty} \leq C \max \left\{1,(\varrho h)^{\varsigma}\right\}
$$

and

$$
\left|\frac{\partial^{m}}{\partial x_{i}^{m}}\left[\omega_{\underline{y}, \varrho}(\underline{x})\right]^{\varsigma}\right| \leq C(\varsigma)\left[\omega_{\underline{y}, \varrho}(\underline{x})\right]^{\varsigma-m} \quad \forall \underline{x} \in \Omega, \forall m \in \mathbb{N}, i \in\{1,2\} ;
$$

where the positive constants $C(\varsigma)$ in $(2.26 \mathrm{a})$ and $(2.26 \mathrm{c})$ depend continuously on $\varsigma$ and are independent of the choice of $\underline{y} \in \Omega$ and $\varrho \geq 1$. It follows immediately from $(2.18 \mathrm{~b})$ and (2.26b) that for all $\sigma \in \mathcal{T}^{h}, \varsigma \in \mathbb{R}, m \in\{0,1\}, i \in\{1,2\}$ and $\eta \in H^{2}(\sigma)$

$$
\int_{\sigma} \omega_{\underline{y}, \varrho}^{\varsigma}\left[\frac{\partial^{m}}{\partial x_{i}^{m}}\left[\left(I-\pi^{h}\right) \eta\right]\right]^{2} \mathrm{~d} \underline{x} \leq C h^{2(2-m)} \int_{\sigma} \omega_{\underline{y}, \varrho}^{\varsigma}\left[\left(\frac{\partial^{2} \eta}{\partial x_{1}^{2}}\right)^{2}+\left(\frac{\partial^{2} \eta}{\partial x_{1} \partial x_{2}}\right)^{2}+\left(\frac{\partial^{2} \eta}{\partial x_{2}^{2}}\right)^{2}\right] \mathrm{d} \underline{x} .
$$

It follows from (2.25), a Hölder inequality and $(2.26 \mathrm{a}, \mathrm{b})$ that for any $p \in(2, \infty), \varsigma>0$ and $\varrho \geq 1$

$$
\begin{aligned}
\left|\underline{\nabla}\left(P^{h} z\right)\right|_{0, p} & \leq C\left[1+\left(\sup _{\underline{y} \in \Omega} \int_{\Omega} \omega_{\underline{y}, \varrho}^{-(\varsigma+2)} \mathrm{d} \underline{x}\right)^{\frac{1}{2}} M_{\varrho, \varsigma}^{h}\right]|\underline{\nabla} z|_{0, p} \\
& \leq C(\varsigma)\left[1+(\varrho h)^{-\frac{\varsigma}{2}} M_{\varrho, \varsigma}^{h}\right]|\underline{\nabla} z|_{0, p} \quad \forall z \in S_{0}^{p},
\end{aligned}
$$

where

$$
M_{\varrho, \varsigma}^{h}:=\max _{i=1,2} \sup _{\underline{y} \in \Omega}\left\{\int_{\Omega} \omega_{\underline{y}, \varrho}^{\varsigma+2}\left|\underline{\nabla}\left(\left[I-P^{h}\right] f_{\underline{y}, i}\right)\right|^{2} \mathrm{~d} \underline{x}\right\}^{\frac{1}{2}} .
$$

The goal is to prove the analogue of [12, Lemma 8.2.6]; that is, for appropriate $\varsigma>0$ and $\varrho$ sufficiently large that there exists an $h_{0}$ such that

$$
M_{\varrho, \varsigma}^{h} \leq C h^{\frac{\varsigma}{2}} \quad \forall h \in\left(0, h_{0}\right) .
$$


It would then follow from (2.28) and (2.30) that (2.22) holds with $\hat{\beta}(p)=C_{1}$ for all $p \in(2, \infty)$, for some constant $C_{1}$. In addition, it would follow from (2.7), (2.21) and the above bound for $p \in(2, \infty)$ that for $p \in\left[\frac{2+\delta}{1+\delta}, 2\right)$ and for all $z \in S_{0}^{p}$

$$
\left|\underline{\nabla}\left(P^{h} z\right)\right|_{0, p} \leq \beta(p) \sup _{0 \neq \eta \in S_{0}^{q}} \frac{\left(\underline{\nabla}\left(P^{h} z\right), \underline{\nabla} \eta\right)}{|\underline{\nabla} \eta|_{0, q}}=\beta(p) \sup _{0 \neq \eta \in S_{0}^{q}} \frac{\left(\underline{\nabla} z, \underline{\nabla}\left(P^{h} \eta\right)\right)}{|\underline{\nabla} \eta|_{0, q}} \leq \beta(p) C_{1}|\underline{\nabla} z|_{0, p},
$$

where $\frac{1}{p}+\frac{1}{q}=1$. As (2.22) trivially holds with $\hat{\beta}(2)=1$ from inspecting (2.21), it follows that (2.22) holds with $\hat{\beta}(p)=C_{2}$ for all $p \in\left[\frac{2+\delta}{1+\delta}, \infty\right)$, for some constant $C_{2}$. Moreover, the desired result $(2.22)$ holds for all $p \in\left[\frac{2+\delta}{1+\delta}, \infty\right)$ by applying the Riesz-Thorin theorem as in Lemma 2.1 to the $P^{h}$ induced mapping that takes $\underline{\nabla} z \in\left[L^{p}(\Omega)\right]^{2}$ to $\underline{\nabla}\left(P^{h} z\right) \in\left[L^{p}(\Omega)\right]^{2}$.

2. Reduction of (2.30) to the weighted regularity bound (2.36). For fixed $y \in$ $\Omega, i \in\{1,2\}$ let $e:=\left(I-P^{h}\right) f_{\underline{y}, i} \in S_{0}, e^{A}:=\left(I-\pi^{h}\right) f_{\underline{y}, i} \in S_{0}$ and $e^{h}:=\left(\pi^{h}-P^{h}\right) f_{\underline{y}, i} \in \bar{S}_{0}^{h}$. It then follows from $(2.21),(2.2),(2.26 \mathrm{~b}, \mathrm{c}),(2.27)$ and $(2.18 \mathrm{a})$ together with assumption $\left(\mathcal{A}_{2}\right)$ for any $\underline{y} \in \Omega, i \in\{1,2\}, \varsigma>0$ and $\varrho \geq 1$ that

$$
\begin{aligned}
\left(\omega_{\underline{y}, \varrho}^{\varsigma+2} \underline{\nabla} e, \underline{\nabla} e\right)= & \left(\underline{\nabla} e, \underline{\nabla}\left(\omega_{\underline{y}, \varrho}^{\varsigma+2} e^{A}\right)+\underline{\nabla}\left(\left(I-\pi^{h}\right)\left[\omega_{\underline{y}, \varrho}^{\varsigma+2} e^{h}\right]\right)\right)-\left(\underline{\nabla} e, e \underline{\nabla} \omega_{\underline{y}, \varrho}^{\varsigma+2}\right) \\
\leq & C(\varsigma)\left[\int_{\Omega}\left[\omega_{\underline{y}, \varrho}^{\varsigma+2}\left|\underline{\nabla} e^{A}\right|^{2}+\omega_{\underline{y}, \varrho}^{\varsigma}\left|e^{A}\right|^{2}\right] \mathrm{d} \underline{x}+\int_{\Omega} \omega_{\underline{y}, \varrho}^{\varsigma}\left|e^{h}\right|^{2} \mathrm{~d} \underline{x}\right. \\
& \left.\quad+\int_{\Omega} \omega_{\underline{y}, \varrho}^{-(\varsigma+2)}\left|\underline{\nabla}\left(\left(I-\pi^{h}\right)\left[\omega_{\underline{y}, \varrho}^{\varsigma+2} e^{h}\right]\right)\right|^{2} \mathrm{~d} \underline{x}\right] \\
\leq & C(\varsigma) \int_{\Omega}\left[\omega_{\underline{y}, \varrho}^{\varsigma+2}\left|\underline{\nabla} e^{A}\right|^{2}+\omega_{\underline{y}, \varrho}^{\varsigma}\left|e^{A}\right|^{2}+\omega_{\underline{y}, \varrho}^{\varsigma}\left|e^{h}\right|^{2}\right] \mathrm{d} \underline{x} .
\end{aligned}
$$

Let $\psi=\mathcal{G}_{0}\left(\omega_{\underline{y}, \varrho}^{\varsigma} e\right)$, where $\mathcal{G}_{0}: S_{0}^{\prime} \times L^{2}\left(\partial_{N} \Omega\right) \rightarrow S_{0}$ is defined as in (A.1). We have, on noting (A.2) and (2.21), that for all $\varsigma>0$

$$
\begin{aligned}
\left(\omega_{\underline{y}, \varrho}^{\varsigma} e, e\right)=(\underline{\nabla} \psi, \underline{\nabla} e) & =\left(\underline{\nabla}\left(\left(I-\pi^{h}\right) \psi\right), \underline{\nabla} e\right) \\
\leq & \varsigma\left(\omega_{\underline{y}, \varrho}^{\varsigma+2} \underline{\nabla} e, \underline{\nabla} e\right)+C \varsigma^{-1} \int_{\Omega} \omega_{\underline{y}, \varrho}^{-(\varsigma+2)}\left|\underline{\nabla}\left(\left(I-\pi^{h}\right) \psi\right)\right|^{2} \mathrm{~d} \underline{x} .
\end{aligned}
$$

It follows from (2.27) and (2.26a) that for any $r \in(1, \infty)$ with $\frac{1}{r}+\frac{1}{r^{\prime}}=1$

$$
\begin{aligned}
& \int_{\Omega} \omega_{\underline{y}, \varrho}^{-(\varsigma+2)}\left|\underline{\nabla}\left(\left(I-\pi^{h}\right) \psi\right)\right|^{2} \mathrm{~d} \underline{x} \leq C h^{2} \sum_{k, \ell=1}^{2} \int_{\Omega} \omega_{\underline{y}, \varrho}^{-(\varsigma+2)}\left|\frac{\partial^{2} \psi}{\partial x_{k} \partial x_{\ell}}\right|^{2} \mathrm{~d} \underline{x} \\
& \leq C h^{2}\left(\int_{\Omega} \omega_{\underline{y}, \varrho}^{-(\varsigma+2) r^{\prime}} \mathrm{d} \underline{x}\right)^{\frac{1}{r^{\prime}}}\|\psi\|_{2,2 r}^{2} \leq C(\varsigma) \varrho^{-2}(\varrho h)^{\frac{2}{r^{\prime}}-\varsigma}\|\psi\|_{2,2 r}^{2} .
\end{aligned}
$$

Next we note that (A.6), (2.1), (2.26a) and (2.26c) yield, on assuming that $\varsigma \in\left(0, \frac{2(r-1)}{r}\right)$,

$$
\begin{aligned}
\|\psi\|_{2,2 r}^{2} & \leq C\left|\omega_{\underline{y}, \varrho}^{\varsigma} e\right|_{0,2 r}^{2} \leq C\left\|\omega_{\underline{y}, \varrho}^{\varsigma} e\right\|_{1, \frac{2 r}{r+1}}^{2} \\
& \leq C\left|\underline{\nabla}\left(\omega_{\underline{y}, \varrho}^{\varsigma} e\right)\right|_{0, \frac{2 r}{r+1}}^{2} \leq C\left(\int_{\Omega} \omega_{\underline{y}, \varrho}^{(\varsigma-2) r} \mathrm{~d} \underline{x}\right)^{\frac{1}{r}} \int_{\Omega} \omega_{\underline{y}, \varrho}^{2-\varsigma}\left|\underline{\nabla}\left(\omega_{\underline{y}, \varrho}^{\varsigma} e\right)\right|^{2} \mathrm{~d} \underline{x} \\
& \leq C(\varsigma)(\varrho h)^{\varsigma-2+\frac{2}{r}}\left[\left(\omega_{\underline{y}, \varrho}^{\varsigma+2} \underline{\nabla} e, \underline{\nabla} e\right)+\left(\omega_{\underline{y}, \varrho}^{\varsigma} e, e\right)\right]
\end{aligned}
$$


where we have noted that $\varsigma \in\left(0, \frac{2(r-1)}{r}\right)$ implies that $(2-\varsigma) r>2$. Therefore for any fixed $\varsigma \in\left(0, \frac{2(r-1)}{r}\right)$, we have for all $\underline{y} \in \Omega, i \in\{1,2\}, \varrho>\varrho_{0}(\varsigma)$ and $h>0$ on combining (2.31), (2.32) with $\varsigma$ sufficiently small, (2.33) and (2.34) that

$$
\left(\omega_{\underline{y}, \varrho}^{\varsigma+2} \underline{\nabla} e, \underline{\nabla} e\right) \leq C(\varsigma, \varrho) \int_{\Omega}\left[\omega_{\underline{y}, \varrho}^{\varsigma+2}\left|\underline{\nabla} e^{A}\right|^{2}+\omega_{\underline{y}, \varrho}^{\varsigma}\left|e^{A}\right|^{2}\right] \mathrm{d} \underline{x} .
$$

Hence the desired result (2.30) follows from (2.29), (2.35) and (2.27); if we can show for any $\underline{y} \in \Omega, i \in\{1,2\}, \varsigma \in(0,1), \varrho \geq 1$ and $h>0$ that

$$
\max _{k, \ell=1,2} \int_{\Omega} \omega_{\underline{y}, \varrho}^{\varsigma+2}\left|\frac{\partial^{2} f_{\underline{y}, i}}{\partial x_{k} \partial x_{\ell}}\right|^{2} \mathrm{~d} \underline{x} \leq C(\varsigma, \varrho) h^{\varsigma-2} .
$$

3. Proof of (2.36). First, we have from (2.26c) that

$$
\max _{k, \ell=1,2} \int_{\Omega} \omega_{\underline{y}, \varrho}^{\varsigma+2}\left|\frac{\partial^{2} f_{\underline{y}, i}}{\partial x_{k} \partial x_{\ell}}\right|^{2} \mathrm{~d} \underline{x} \leq C(\varsigma)\left[\left|\omega_{\underline{y}, \varrho}^{\frac{\varsigma}{2}+1} f_{\underline{y}, i}\right|_{2}^{2}+\int_{\Omega}\left[\omega_{\underline{y}, \varrho}^{\varsigma}\left|\underline{\nabla} f_{\underline{y}, i}\right|^{2}+\omega_{\underline{y}, \varrho}^{\varsigma-2}\left|f_{\underline{y}, i}\right|^{2}\right] \mathrm{d} \underline{x}\right] .
$$

Second, it follows from $(2.24)$ that $\omega_{\underline{y}, \varrho}^{\frac{\varsigma}{2}+1} f_{\underline{y}, i} \in S_{0}$ solves for all $\eta \in S_{0}$

$$
\begin{gathered}
\left(\underline{\nabla}\left(\omega_{\underline{\underline{y}}, \varrho}^{\frac{\varsigma}{2}+1} f_{\underline{y}, i}\right), \underline{\nabla} \eta\right)=\left(\underline{\nabla}\left(f_{\underline{y}, i}\right), \underline{\nabla}\left(\omega_{\underline{y}, \varrho}^{\frac{\varsigma}{2}+1} \eta\right)\right)+\left(f_{\underline{y}, i \underline{\nabla}}\left(\omega_{\underline{y}, \varrho}^{\frac{\varsigma}{2}+1}\right), \underline{\nabla} \eta\right)-\left(\underline{\nabla} f_{\underline{y}, i}, \underline{\nabla}\left(\omega_{\underline{\underline{y}}, \varrho}^{\frac{\varsigma}{2}+1}\right) \eta\right) \\
=-\left(\left(\underline{\nabla} \delta_{\underline{y}}^{h}\right)_{i}, \omega_{\underline{y}, \varrho}^{\frac{\varsigma}{2}+1} \eta\right)-\left(\underline{\nabla}\left(f_{\underline{y}, i}\right), \underline{\nabla}\left(\omega_{\underline{\underline{y}}, \varrho}^{\frac{\varsigma}{2}+1}\right) \eta\right)-\left(\underline{\nabla} \cdot\left(f_{\underline{y}, i} \underline{\nabla}\left(\omega_{\underline{y}, \varrho}^{\frac{\varsigma}{2}+1}\right)\right), \eta\right) \\
+\int_{\partial_{N} \Omega} f_{\underline{y}, i} \frac{\partial}{\partial \underline{\underline{\nu}} \partial \Omega}\left(\omega_{\underline{y}, \varrho}^{\frac{\varsigma}{2}+1}\right) \eta \mathrm{d} s .
\end{gathered}
$$

Noting (A.1), $f_{\underline{y}, i}=0$ on $\overline{\partial_{D} \Omega} \cap \overline{\partial_{N} \Omega}, f_{\underline{y}, i} \in H^{\frac{1}{2}}\left(\partial_{N} \Omega\right)$ as $f_{\underline{y}, i} \in H^{2}(\Omega) \subset C(\bar{\Omega})$ and $\omega_{\underline{y}, p}^{\frac{\varsigma}{2}+1} \in C^{\infty}(\bar{\Omega})$, and applying the bounds (A.5), (2.26c) and the trace inequality $\|$. $\left\|_{\frac{1}{2}, \partial_{N} \Omega} \leq C\right\| \cdot \|_{1, \Omega}$ to $(2.38)$ yields that

$$
\begin{aligned}
\left|\omega_{\underline{y}, \varrho}^{\frac{\varsigma}{2}+1} f_{\underline{y}, i}\right|_{2} & \leq C\left[\left|\omega_{\underline{y}, \varrho}^{\frac{\varsigma}{2}+1} \underline{\nabla} \delta_{\underline{y}}^{h}\right|_{0}+\left|\omega_{\underline{y}, \varrho}^{\frac{\varsigma}{2}} \underline{\nabla} f_{\underline{y}, i}\right|_{0}+\left|\omega_{\underline{y}, \varrho}^{\frac{\varsigma}{2}-1} f_{\underline{y}, i}\right|_{0}+\left|f_{\underline{y}, i} \frac{\partial}{\partial \underline{\nu}_{\partial \Omega}}\left(\omega_{\underline{y}, \varrho}^{\frac{\varsigma}{2}+1}\right)\right|_{\frac{1}{2}, \partial_{N} \Omega}\right] \\
& \leq C\left[\left|\omega_{\underline{\underline{y}}, \varrho}^{\frac{\varsigma}{2}+1} \underline{\nabla} \delta_{\underline{y}}^{h}\right|_{0}+\left|\omega_{\underline{\underline{2}}, \varrho}^{\frac{\varsigma}{2}} \underline{\nabla} f_{\underline{y}, i}\right|_{0}+\left|\omega_{\underline{y}, \varrho}^{\frac{\varsigma}{2}-1} f_{\underline{y}, i}\right|_{0}\right] .
\end{aligned}
$$

It holds that

$$
\left(\omega_{\underline{y}, \varrho}^{\varsigma} \underline{\nabla} f_{\underline{y}, i}, \underline{\nabla} f_{\underline{y}, i}\right)=\left(\underline{\nabla} f_{\underline{y}, i}, \underline{\nabla}\left(\omega_{\underline{y}, \varrho}^{\varsigma} f_{\underline{y}, i}\right)\right)-\left(f_{\underline{y}, i} \underline{\nabla} \omega_{\underline{y}, \varrho}^{\varsigma}, \underline{\nabla} f_{\underline{y}, i}\right) .
$$

Similarly to (2.38), choosing $\eta=\omega_{\underline{y}, \varrho}^{\varsigma} f_{\underline{y}, i} \in S_{0}$ in (2.24) yields that

$$
\left(\underline{\nabla} f_{\underline{y}, i}, \underline{\nabla}\left(\omega_{\underline{y}, \varrho}^{\varsigma} f_{\underline{y}, i}\right)\right)=-\left(\left(\underline{\nabla} \delta_{\underline{y}}^{h}\right)_{i}, \omega_{\underline{y}, \varrho}^{\varsigma} f_{\underline{y}, i}\right) .
$$

Combining (2.37), (2.39), (2.40) and (2.41) yields that

$$
\max _{k, \ell=1,2} \int_{\Omega} \omega_{\underline{y}, \varrho}^{\varsigma+2}\left|\frac{\partial^{2} f_{\underline{y}, i}}{\partial x_{k} \partial x_{\ell}}\right|^{2} \mathrm{~d} \underline{x} \leq C(\varsigma)\left[\left|\omega_{\underline{y}, \varrho}^{\frac{\varsigma}{2}-1} f_{\underline{y}, i}\right|_{0}^{2}+\left|\omega_{\underline{y}, \varrho}^{\frac{\varsigma}{2}+1} \underline{\nabla} \delta_{\underline{y}}^{h}\right|_{0}^{2}\right] .
$$


For $p \in\left(1, \frac{2}{\varsigma}\right)$, let $\xi=\operatorname{sign}\left(f_{\underline{y}, i}\right)\left|f_{\underline{y}, i}\right|^{2 p-1}, \ell=1,2$. It follows from (A.2), (2.24), (2.1), (A.6) and (2.26a) that

$$
\begin{aligned}
& \left|f_{\underline{y}, i}\right|_{0,2 p}^{2 p}=\left(\xi, f_{\underline{y}, i}\right)=\left(\underline{\nabla}\left(\mathcal{G}_{0} \xi\right), \underline{\nabla}\left(f_{\underline{y}, i}\right)\right)=\left(\delta_{\underline{y}}^{h},\left[\underline{\nabla}\left(\mathcal{G}_{0} \xi\right)\right]_{i}\right) \\
& \leq C\left|\delta_{\underline{y}}^{h}\right|_{0, \frac{2 p}{p+1}}\left|\mathcal{G}_{0} \xi\right|_{1, \frac{2 p}{p-1}} \leq C\left|\delta_{\underline{y}}^{h}\right|_{0, \frac{2 p}{p+1}}\left\|\mathcal{G}_{0} \xi\right\|_{2, \frac{2 p}{2 p-1}} \\
& \leq C\left|\delta_{\underline{y}}^{h}\right|_{0, \frac{2 p}{p+1}}|\xi|_{0, \frac{2 p}{2 p-1}} \leq C\left|\delta_{\underline{y}}^{h}\right|_{0, \frac{2 p}{p+1}}\left|f_{\underline{y}, i}\right|_{0,2 p}^{2 p-1} \leq C\left|\delta_{\underline{y}}^{h}\right|_{0, \frac{2 p}{p+1}}^{2 p} \\
& \leq C\left(\int_{\Omega} \omega_{\underline{y}, \varrho}^{-(\varsigma+2) p} \mathrm{~d} \underline{x}\right)\left|\omega_{\underline{y}, \varrho}^{\frac{\varsigma}{2}+1} \delta_{\underline{y}}^{h}\right|_{0}^{2 p} \leq C(\varsigma)(\varrho h)^{2-(\varsigma+2) p}\left|\omega_{\underline{y}, \varrho}^{\frac{\varsigma}{2}+1} \delta_{\underline{y}}^{h}\right|_{0}^{2 p} .
\end{aligned}
$$

Next we have from (2.26a) that for $p \in\left(1, \frac{2}{\varsigma}\right)$ with $\frac{1}{p}+\frac{1}{p^{\prime}}=1$

$$
\left|\omega_{\underline{y}, \varrho}^{\frac{\varsigma}{2}-1} f_{\underline{y}, i}\right|_{0}^{2} \leq C\left(\int_{\Omega} \omega_{\underline{y}, \varrho}^{(\varsigma-2) p^{\prime}} \mathrm{d} \underline{x}\right)^{\frac{1}{p^{\prime}}}\left|f_{\underline{y}, i}\right|_{0,2 p}^{2} \leq C(\varsigma)(\varrho h)^{\varsigma-\frac{2}{p}}\left|f_{\underline{y}, i}\right|_{0,2 p}^{2},
$$

where we have noted that $p \in\left(1, \frac{2}{\varsigma}\right)$ implies that $(\varsigma-2) p^{\prime}<-2$. Finally, combining (2.42), (2.44), (2.43), (2.23) and (2.26a) yields that

$$
\max _{k, \ell=1,2} \int_{\Omega} \omega_{\underline{y}, \varrho}^{\varsigma+2}\left|\frac{\partial^{2} f_{\underline{y}, i}}{\partial x_{k} \partial x_{\ell}}\right|^{2} \mathrm{~d} \underline{x} \leq C(\varsigma, \varrho) h^{-2}\left|\omega_{\underline{y}, \varrho}^{\frac{\varsigma}{2}+1} \delta_{\underline{y}}^{h}\right|_{0}^{2} \leq C(\varsigma, \varrho) h^{-6}\left|\omega_{\underline{\underline{y}}, \varrho}^{\frac{\varsigma}{2}+1}\right|_{0, \sigma_{\underline{y}}}^{2} \leq C(\varsigma, \varrho) h^{\varsigma-2}
$$

and hence the desired result (2.36).

We now have a discrete analogue of a result similar to (2.7).

Lemma 2.3. Let $d=2$ and the remaining assumptions of Lemma 2.2 hold. Then there exists $\delta_{1} \in(0, \delta)$ and $C>0$ such that for all $p \in\left[2,2+\delta_{1}\right]$ and for all $h \in\left(0, h_{0}\right)$,

$$
\left|\underline{\nabla} z^{h}\right|_{0, p} \leq C \sup _{0 \neq \chi \in S_{0}^{h}} \frac{\left(c\left(\underline{x}, u^{h}\right) \underline{\nabla} z^{h}, \underline{\nabla} \chi\right)}{|\underline{\nabla} \chi|_{0, q}} \quad \forall z^{h} \in S_{0}^{h}, \quad \forall u^{h} \in K_{f}^{h},
$$

where $\frac{1}{p}+\frac{1}{q}=1$.

Proof The proof is an adaptation of that given in [4, Lemma 2.4], which was itself adapted from [12, Chapter 8.6].

It follows from $(2.7),(2.21)$ and $(2.22)$ that for all $p \in[2,2+\delta]$, for all $h \in\left(0, h_{0}\right)$ and for all $z^{h} \in S_{0}^{h} \subset S_{0}^{p}$,

$$
\begin{aligned}
\left|\underline{\nabla} z^{h}\right|_{0, p} & \leq \beta(p) \sup _{0 \neq \eta \in S_{0}^{q}} \frac{\left(\underline{\nabla} z^{h}, \underline{\nabla} \eta\right)}{|\underline{\nabla} \eta|_{0, q}} \leq \beta(p) \widehat{\beta}(q) \sup _{0 \neq \eta \in S_{0}^{q}} \frac{\left(\underline{\nabla} z^{h}, \underline{\nabla}\left(P^{h} \eta\right)\right)}{\left|\underline{\nabla}\left(P^{h} \eta\right)\right|_{0, q}} \\
& \leq(1+\varsigma(p)) \sup _{0 \neq \chi \in S_{0}^{h}} \frac{\left(\underline{\nabla} z^{h}, \underline{\nabla} \chi\right)}{|\underline{\nabla} \chi|_{0, q}},
\end{aligned}
$$


where $\varsigma \in C([2,2+\delta])$, $\varsigma(p) \geq 0$ and $\varsigma(p) \rightarrow 0$ as $p \rightarrow 2$. On recalling (1.5a) and (1.6) we define $B(z, \eta)=\left(\left(1-\frac{c\left(\underline{x}, \theta^{h}\right)}{c_{\max }}\right) \underline{\nabla} z, \underline{\nabla} \eta\right)$ for all $z \in S_{0}^{p}, \eta \in S_{0}^{q}$ and $\theta^{h} \in K_{f}^{h}$. It follows from (1.5a) and (1.6) that

$$
|B(z, \eta)| \leq\left(1-\frac{c_{\min }}{c_{\max }}\right)|\underline{\nabla} z|_{0, p}|\underline{\nabla} \eta|_{0, q} .
$$

Combining (2.46) and (2.47) yields for all $z^{h} \in S_{0}^{h}$ and $\theta^{h} \in K_{f}^{h}$ that

$$
\begin{aligned}
& \frac{1}{1+\varsigma(p)}\left|\underline{\nabla} z^{h}\right|_{0, p} \leq \sup _{0 \neq \chi \in S_{0}^{h}} \frac{B\left(z^{h}, \chi\right)+\left[c_{\max }\right]^{-1}\left(c\left(\underline{x}, \theta^{h}\right) \underline{\nabla} z^{h}, \underline{\nabla} \chi\right)}{|\underline{\nabla} \chi|_{0, q}} \\
& \leq\left(1-\frac{c_{\min }}{c_{\max }}\right)\left|\underline{\nabla} z^{h}\right|_{0, p}+\frac{1}{c_{\max }} \sup _{\substack{0 \neq \chi \in S_{0}^{h}\\
}} \frac{\left(c\left(\underline{x}, \theta^{h}\right) \underline{\nabla} z^{h}, \underline{\nabla} \chi\right)}{|\underline{\nabla} \chi|_{0, q}}
\end{aligned}
$$

which implies that

$$
\left[\frac{1}{1+\varsigma(p)}-\left(1-\frac{c_{\min }}{c_{\max }}\right)\right]\left|\underline{\nabla} z^{h}\right|_{0, p} \leq \frac{1}{c_{\max }} \sup _{0 \neq \chi \in S_{0}^{h}} \frac{\left(c\left(\underline{x}, \theta^{h}\right) \underline{\nabla} z^{h}, \underline{\nabla} \chi\right)}{|\underline{\nabla} \chi|_{0, q}} .
$$

Since $\varsigma(p) \rightarrow 0$ as $p \rightarrow 2$ and as $\varsigma$ is continuous, one can choose $\delta_{1} \in(0, \delta)$ such that $\varsigma(p) \leq \frac{1}{2} \frac{c_{\min }}{c_{\max }-c_{\min }}$ for all $p \in\left[2,2+\delta_{1}\right]$. Hence $(2.47)$ yields the result $(2.45)$.

\section{Finite element approximation}

In this section we present our finite element approximation of (1.1a-f), and prove stability for $d=2$ and $d=3$. This will enable us to also prove a convergence result in the case $d=2$ in the next section.

In addition to $\mathcal{T}^{h}$, let $0=t_{0}<t_{1}<\cdots<t_{N-1}<t_{N}=T$ be a partitioning of $[0, T]$ into possibly variable time-steps $\tau_{n}:=t_{n}-t_{n-1}, n=1 \rightarrow N$. We set $\tau:=\max _{n=1 \rightarrow N} \tau_{n}$. We then propose the following fully practical finite element approximation of $(1.1 \mathrm{a}-\mathrm{f})$. Given $U^{0} \in K_{f}^{h}$, for $n \geq 1$ find $\left(\Phi^{n}, U^{n}, W^{n}\right) \in S_{g}^{h} \times K_{f}^{h} \times S_{f}^{h}$ such that

$$
\begin{aligned}
& \left(c\left(\underline{x}, U^{n}\right) \underline{\nabla} \Phi^{n}, \underline{\nabla} \chi\right)=0 \quad \forall \chi \in S_{0}^{h}, \\
& \gamma\left(\frac{U^{n}-U^{n-1}}{\tau_{n}}, \chi\right)_{\Omega_{f}}^{h}+\left(\underline{\nabla} W^{n}, \underline{\nabla} \chi\right)_{\Omega_{f}}=0 \quad \forall \chi \in S_{f}^{h}, \\
& \gamma\left(\underline{\nabla} U^{n}, \underline{\nabla}\left(\chi-U^{n}\right)\right)_{\Omega_{f}} \geq\left(W^{n}+\gamma^{-1} U^{n-1}, \chi-U^{n}\right)_{\Omega_{f}}^{h}+\frac{1}{2} \alpha c_{f}^{\prime}\left(\left|\underline{\nabla} \Phi^{n}\right|^{2}, \chi-U^{n}\right)_{\Omega_{f}} \\
& \forall \chi \in K_{f}^{h} .
\end{aligned}
$$

The presence of $U^{n}$ in the diffusion coefficient in (3.1a) means that the scheme $(3.1 \mathrm{a}-\mathrm{c})$ is coupled. Here we note from (1.5a) that $c\left(\underline{x}, U^{n}\right) \equiv c_{0}+c_{1} \in \mathbb{R}_{>0}$ in $\bar{\Omega} \backslash \overline{\Omega_{f}}$, and so no extension of $U^{n}$ is required for (3.1a). Furthermore, $\left|\underline{\nabla} \Phi^{n}\right|^{2}$ is restricted to $\Omega_{f}$ in (3.1c).

Assuming the existence of a solution $\left\{\left(\Phi^{n}, U^{n}, W^{n}\right)\right\}_{n=1}^{N}$ to $(3.1 \mathrm{a}-\mathrm{c})$, we can establish the following results, where for mathematical convenience we define $\Phi^{0} \in S_{g}^{h}$ such that

$$
\left(c\left(\underline{x}, U^{0}\right) \underline{\nabla} \Phi^{0}, \underline{\nabla} \chi\right)=0 \quad \forall \chi \in S_{0}^{h} .
$$


Lemma 3.1. Let the assumptions $\left(\mathcal{A}_{1}\right)$ hold and let $\left\{\left(\Phi^{n}, U^{n}, W^{n}\right)\right\}_{n=1}^{N}$ be a solution to $(3.1 \mathrm{a}-\mathrm{c})$. Then it holds for $n=1 \rightarrow N$ that

$$
\left(U^{n}, 1\right)_{\Omega_{f}}=\left(U^{n-1}, 1\right)_{\Omega_{f}} .
$$

If, in addition, $\Phi^{0} \in S_{g}^{h}$ satisfies (3.2), then it holds for $n=0 \rightarrow N$ that

$$
\left(c\left(\underline{x}, U^{n}\right) \underline{\nabla} \Phi^{n}, \underline{\nabla} \Phi^{n}\right) \leq\left(c\left(\underline{x}, U^{n}\right), 1\right)
$$

and

$$
g^{-} \leq \Phi^{n} \leq g^{+} \quad \text { in } \bar{\Omega},
$$

where $g^{ \pm} \in \mathbb{R}$ are defined as in (1.2). Moreover, there exists a constant $C>0$ such that, for $n=0 \rightarrow N$,

$$
\left|\Phi^{n}\right|_{1} \leq C
$$

Proof The desired result (3.3) follows immediately on choosing $\chi=1$ in (3.1b).

Choosing $\chi=\Phi^{n}-x_{1} \in S_{0}^{h}$ in (3.1a) and (3.2), on noting Hölder's inequality and (2.2), yields that

$$
\left(c\left(\underline{x}, U^{n}\right) \underline{\nabla} \Phi^{n}, \underline{\nabla} \Phi^{n}\right) \leq \frac{1}{2}\left(c\left(\underline{x}, U^{n}\right),\left|\underline{\nabla} \Phi^{n}\right|^{2}\right)+\frac{1}{2}\left(c\left(\underline{x}, U^{n}\right),\left|\underline{\nabla} x_{1}\right|^{2}\right),
$$

which gives the desired result (3.4).

The proof of the discrete maximum result (3.5) is motivated by the proof of $[7$, Lemma 2.2]. It follows from (2.16) that

$$
\left(\underline{\nabla} \pi^{h}[\chi]_{+}, \underline{\nabla} \pi^{h}[\chi]_{+}\right) \leq\left(\underline{\nabla} \chi, \underline{\nabla} \pi^{h}[\chi]_{+}\right) \quad \forall \chi \in S^{h}
$$

where $[s]_{+}:=\max \{s, 0\}$ for all $s \in \mathbb{R}$. This implies, on choosing choosing $\chi=\pi^{h}\left[\Phi^{n}-\right.$ $\left.g^{+}\right]_{+} \in S_{0}^{h}$ in (3.1a) and (3.2), for $n=0$, that

$$
\begin{aligned}
\left(c\left(\underline{x}, U^{n}\right),\left|\underline{\nabla} \pi^{h}\left[\Phi^{n}-g^{+}\right]_{+}\right|^{2}\right) & \leq\left(c\left(\underline{x}, U^{n}\right) \underline{\nabla} \pi^{h}\left[\Phi^{n}-g^{+}\right], \underline{\nabla} \pi^{h}\left[\Phi^{n}-g^{+}\right]_{+}\right) \\
& \leq\left(c\left(\underline{x}, U^{n}\right) \underline{\nabla} \Phi^{n}, \underline{\nabla} \pi^{h}\left[\Phi^{n}-g^{+}\right]_{+}\right)=0 .
\end{aligned}
$$

Hence it follows from (1.6) that $\underline{\nabla} \pi^{h}\left[\Phi^{n}-g^{+}\right]_{+}=0$, and so $\pi^{h}\left[\Phi^{n}-g^{+}\right]_{+}=0$, i.e. $\Phi^{n} \leq g^{+}$. Similarly, choosing $\chi=\pi^{h}\left[g^{-}-\Phi^{n}\right]_{+} \in S_{0}^{h}$ in (3.1a) and (3.2) we get that $\Phi^{n} \geq g^{-}$, and hence the desired result (3.5).

Finally, on recalling (1.6), it immediately follows from (3.4) that $\left|\Phi^{n}\right|_{1} \leq \frac{c_{\max }}{c_{\min }}|\Omega|$, and hence we have the desired result (3.6).

Let

$$
\mathcal{J}(\chi, \eta)=\frac{1}{2}\left\{\gamma|\chi|_{1, \Omega_{f}}^{2}-\gamma^{-1}|\chi|_{h, \Omega_{f}}^{2}\right\}-\frac{1}{2} \alpha\left(c(\underline{x}, \chi),|\underline{\nabla} \eta|^{2}\right) \quad \chi \in K_{f}^{h}, \eta \in S^{h}
$$


Lemma 3.2. Let the assumptions $\left(\mathcal{A}_{1}\right)$ hold and $U^{0} \in K_{f}^{h}$. Let $\left\{\left(\Phi^{n}, U^{n}, W^{n}\right)\right\}_{n=1}^{N}$ be a solution to $(3.1 \mathrm{a}-\mathrm{c})$. Then it holds for $n=1 \rightarrow N$ that

$$
\mathcal{J}\left(U^{n-1}, \Phi^{n}\right)=\mathcal{J}\left(U^{n-1}, \Phi^{n-1}\right)-\frac{1}{2} \alpha\left(c\left(\underline{x}, U^{n-1}\right),\left|\underline{\nabla}\left(\Phi^{n}-\Phi^{n-1}\right)\right|^{2}\right),
$$

and

$$
\mathcal{J}\left(U^{n}, \Phi^{n}\right)+\frac{1}{2} \gamma\left|U^{n}-U^{n-1}\right|_{1, \Omega_{f}}^{2}+\frac{1}{2} \gamma^{-1}\left|U^{n}-U^{n-1}\right|_{h, \Omega_{f}}^{2}+\frac{\tau_{n}}{\gamma}\left|W^{n}\right|_{1, \Omega_{f}}^{2} \leq \mathcal{J}\left(U^{n-1}, \Phi^{n}\right) .
$$

In addition, there exists a constant $C>0$ such that

$$
\mathcal{J}\left(U^{n}, \Phi^{n}\right) \geq-C
$$

for $n=0 \rightarrow N$.

Proof Choosing $\chi=\Phi^{n}-\Phi^{n+1} \in S_{0}^{h}$ in (3.1a) for $n=1 \rightarrow N-1$, and $\chi=\Phi^{0}-\Phi^{1} \in S_{0}^{h}$ in (3.2), and applying the identity

$$
r(r-s)=\frac{1}{2} r^{2}+\frac{1}{2}(r-s)^{2}-\frac{1}{2} s^{2}
$$

yields

$$
\left(c\left(\underline{x}, U^{n-1}\right),\left|\underline{\nabla} \Phi^{n-1}\right|^{2}+\left|\underline{\nabla}\left(\Phi^{n-1}-\Phi^{n}\right)\right|^{2}\right)=\left(c\left(\underline{x}, U^{n-1}\right),\left|\underline{\nabla} \Phi^{n}\right|^{2}\right),
$$

for $n=1 \rightarrow N$. Therefore, on noting (3.8), we have the desired result (3.9). In order to obtain the bound (3.10), we first note that it follows from $c$ being affine linear, recall $(1.5 \mathrm{a}, \mathrm{b})$, that $c^{\prime}\left(\underline{x}, U^{n-1}\right)\left[U^{n}-U^{n-1}\right]=c\left(\underline{x}, U^{n}\right)-c\left(\underline{x}, U^{n-1}\right)$ for all $\underline{x} \in \Omega$, and therefore that, for $n=1 \rightarrow N$,

$$
\begin{gathered}
\frac{1}{2} \alpha c_{f}^{\prime}\left(\left|\underline{\nabla} \Phi^{n}\right|^{2}, U^{n-1}-U^{n}\right)_{\Omega_{f}}=\frac{1}{2} \alpha\left(c^{\prime}\left(\underline{x}, U^{n-1}\right)\left|\underline{\nabla} \Phi^{n}\right|^{2}, U^{n-1}-U^{n}\right) \\
=-\frac{1}{2} \alpha\left(c\left(\underline{x}, U^{n}\right),\left|\underline{\nabla} \Phi^{n}\right|^{2}\right)+\frac{1}{2} \alpha\left(c\left(\underline{x}, U^{n-1}\right),\left|\underline{\nabla} \Phi^{n}\right|^{2}\right) .
\end{gathered}
$$

Choosing $\chi=W^{n}$ in (3.1b) and $\chi=U^{n-1}$ in (3.1c) yields, for $n=1 \rightarrow N$, that

$$
\begin{aligned}
\gamma(\underline{\nabla} & \left.U^{n}, \underline{\nabla}\left(U^{n-1}-U^{n}\right)\right)_{\Omega_{f}} \\
& \geq\left(W^{n}+\gamma^{-1} U^{n-1}, U^{n-1}-U^{n}\right)_{\Omega_{f}}^{h}+\frac{1}{2} \alpha c_{f}^{\prime}\left(\left|\underline{\nabla} \Phi^{n}\right|^{2}, U^{n-1}-U^{n}\right)_{\Omega_{f}} \\
& =\frac{\tau_{n}}{\gamma}\left(\underline{\nabla} W^{n}, \underline{\nabla} W^{n}\right)_{\Omega_{f}}+\gamma^{-1}\left(U^{n-1}, U^{n-1}-U^{n}\right)_{\Omega_{f}}^{h}+\frac{1}{2} \alpha c_{f}^{\prime}\left(\left|\underline{\nabla} \Phi^{n}\right|^{2}, U^{n-1}-U^{n}\right)_{\Omega_{f}} .
\end{aligned}
$$

On combining (3.14) and (3.13), and on recalling (3.12), we obtain, for $n=1 \rightarrow N$, that

$$
\begin{gathered}
\frac{1}{2} \gamma\left|U^{n-1}\right|_{1, \Omega_{f}}^{2}-\frac{1}{2} \gamma\left|U^{n}\right|_{1, \Omega_{f}}^{2}-\frac{1}{2} \gamma\left|U^{n}-U^{n-1}\right|_{1, \Omega_{f}}^{2}-\frac{1}{2} \alpha\left(c\left(\underline{x}, U^{n-1}\right),\left|\underline{\nabla} \Phi^{n}\right|^{2}\right) \\
\geq \frac{\tau_{n}}{\gamma}\left|W^{n}\right|_{1, \Omega_{f}}^{2}+\frac{1}{2} \gamma^{-1}\left|U^{n-1}\right|_{h, \Omega_{f}}^{2}-\frac{1}{2} \gamma^{-1}\left|U^{n}\right|_{h, \Omega_{f}}^{2} \\
+\frac{1}{2} \gamma^{-1}\left|U^{n}-U^{n-1}\right|_{h, \Omega_{f}}^{2}-\frac{1}{2} \alpha\left(c\left(\underline{x}, U^{n}\right),\left|\underline{\nabla} \Phi^{n}\right|^{2}\right) .
\end{gathered}
$$

Rearranging, and taking note of (3.8) we get the second energy property (3.10). Finally, it immediately follows from $U^{n} \in K_{f}^{h}$, for $n=0 \rightarrow N$, and (3.6) that the desired result (3.11) holds. 


\subsection{Existence of a discrete solution}

We consider a standard fixed point approach to prove existence of a solution to $(3.1 \mathrm{a}-\mathrm{c})$.

Given $U^{n-1} \in K_{f}^{h}$ for any $n=1 \rightarrow N$, let $U^{n, 0}=U^{n-1}$. Then for any $i \geq 1$, find $\Phi^{n, i} \in S_{g}^{h}$ such that

$$
\left(c\left(\underline{x}, U^{n, i-1}\right) \underline{\nabla} \Phi^{n, i}, \underline{\nabla} \chi\right)=0 \quad \forall \chi \in S_{0}^{h},
$$

and then find $\left(U^{n, i}, W^{n, i}\right) \in K_{f}^{h} \times S_{f}^{h}$ such that

$$
\begin{aligned}
& \gamma\left(\frac{U^{n, i}-U^{n-1}}{\tau_{n}}, \chi\right)_{\Omega_{f}}^{h}+\left(\underline{\nabla} W^{n, i}, \underline{\nabla} \chi\right)_{\Omega_{f}}=0 \quad \forall \chi \in S_{f}^{h}, \\
& \gamma\left(\underline{\nabla} U^{n, i}, \underline{\nabla}\left(\chi-U^{n, i}\right)\right)_{\Omega_{f}} \geq\left(W^{n, i}+\gamma^{-1} U^{n-1}, \chi-U^{n, i}\right)_{\Omega_{f}}^{h}+\frac{1}{2} \alpha c_{f}^{\prime}\left(\left|\underline{\nabla} \Phi^{n, i}\right|^{2}, \chi-U^{n, i}\right)_{\Omega_{f}} \\
& \forall \chi \in K_{f}^{h} .
\end{aligned}
$$

We introduce the linear operator $\mathcal{G}_{f}^{h}: Z_{f}^{h} \rightarrow Z_{f}^{h}$, defined such that

$$
\left(\underline{\nabla} \mathcal{G}_{f}^{h} \psi, \underline{\nabla} \chi\right)_{\Omega_{f}}=(\psi, \chi)_{\Omega_{f}}^{h} \quad \forall \chi \in S_{f}^{h}
$$

where

$$
Z_{f}^{h}:=\left\{z^{h} \in S_{f}^{h}:\left(z^{h}, 1\right)_{\Omega_{f}}=0\right\}
$$

and let

$$
K_{f}^{h}(0):=\left\{\chi \in K_{f}^{h}: \chi-U^{0} \in Z_{f}^{h}\right\} .
$$

We note that it immediately follows from (3.3) that $U^{n} \in K_{f}^{h}(0)$ for $n=1 \rightarrow N$. Establishing the existence of a solution to $(3.16 \mathrm{a}-\mathrm{c})$ is straightforward, see also [3].

Lemma 3.3. Let the assumptions $\left(\mathcal{A}_{1}\right)$ hold and $U^{n, i-1} \in K_{f}^{h}(0)$. Then there exists a solution $\left(\Phi^{n, i}, U^{n, i}, W^{n, i}\right) \in S_{g}^{h} \times K_{f}^{h}(0) \times S_{f}^{h}$ to $(3.16 \mathrm{a}-\mathrm{c})$, with $\Phi^{n, i}$ and $U^{n, i}$ being unique.

Proof Since (3.16a) is a linear finite dimensional system, the existence of a solution $\Phi^{n, i} \in S_{g}^{h}$ follows immediately from uniqueness, which is given by a Friedrich's inequality.

It follows from (3.16b) and (3.17) that $W^{n, i}$ can be written as

$$
W^{n, i}=-\gamma \mathcal{G}_{f}^{h}\left[\frac{U^{n, i}-U^{n-1}}{\tau_{n}}\right]+\lambda^{n, i},
$$

where $\lambda^{n, i} \in \mathbb{R}$. Hence $(3.16 \mathrm{~b}, \mathrm{c})$ can be reduced to: Find $U^{n, i} \in K_{f}^{h}(0)$ such that

$$
\begin{aligned}
\gamma\left(\underline{\nabla} U^{n, i},\right. & \left.\underline{\nabla}\left(\chi-U^{n, i}\right)\right)_{\Omega_{f}}+\gamma\left(\mathcal{G}_{f}^{h}\left[\frac{U^{n, i}-U^{n-1}}{\tau_{n}}\right], \chi-U^{n, i}\right)_{\Omega_{f}}^{h} \\
& \geq \gamma^{-1}\left(U^{n-1}, \chi-U^{n, i}\right)_{\Omega_{f}}^{h}+\frac{1}{2} \alpha c_{f}^{\prime}\left(\left|\underline{\nabla} \Phi^{n, i}\right|^{2}, \chi-U^{n, i}\right)_{\Omega_{f}} \quad \forall \chi \in K_{f}^{h}(0) .
\end{aligned}
$$


We know that there exists a unique $U^{n, i} \in K_{f}^{h}(0)$ solving (3.19) since, on noting (3.17), this is the Euler-Lagrange variational inequality of the strictly convex minimisation problem

$$
\min _{z_{h} \in K_{f}^{h}(0)}\left\{\frac{\gamma}{2}\left|z^{h}\right|_{1, \Omega_{f}}^{2}+\frac{\gamma}{2 \tau_{n}}\left|\underline{\nabla} \mathcal{G}_{f}^{h}\left(z^{h}-U^{n-1}\right)\right|_{0, \Omega_{f}}^{2}-\gamma^{-1}\left(U^{n-1}, z^{h}\right)_{\Omega_{f}}^{h}-\frac{1}{2} \alpha c_{f}^{\prime}\left(\left|\underline{\nabla} \Phi^{n, i}\right|^{2}, z^{h}\right)_{\Omega_{f}}\right\} .
$$

Existence of the Lagrange multiplier $\lambda^{n, i}$ in (3.18) then follows from standard optimisation theory, see e.g. [15].

Theorem 3.4. Let the assumptions $\left(\mathcal{A}_{1}\right)$ hold and $U^{n-1} \in K_{f}^{h}(0)$. Then for all $h, \tau_{n}>0$, there exists a solution $\left(\Phi^{n}, U^{n}, W^{n}\right) \in S_{g}^{h} \times K_{f}^{h}(0) \times S_{f}^{h}$ to the $n^{\text {th }}$ step of $(3.1 \mathrm{a}-\mathrm{c})$.

Proof By Lemma 3.3 we have existence and uniqueness of solutions $\left(\Phi^{n, i}, U^{n, i}\right)$ to $(3.16 \mathrm{a}-$ c), for fixed $h, \tau_{n}>0$ and for all $i \geq 1$. Referring to (3.16a), (3.19), we write $U^{n, i}=$ $F\left(U^{n, i-1}\right)$, where $F: K_{f}^{h}(0) \rightarrow K_{f}^{h}(0)$. Lemma 3.3 implies that $F$ is well-defined.

Repeating the arguments in the proof of Lemma 3.3, we see that $(3.1 \mathrm{a}-\mathrm{c})$ can equivalently be rewritten as: Find $\left(\Phi^{n}, U^{n}, W^{n}\right) \in S_{g}^{h} \times K_{f}^{h}(0) \times S_{f}^{h}$ such that

$$
\begin{aligned}
& \left(c\left(\underline{x}, U^{n}\right) \underline{\nabla} \Phi^{n}, \underline{\nabla} \chi\right)=0 \quad \forall \chi \in S_{0}^{h}, \\
& \gamma\left(\underline{\nabla} U^{n}, \underline{\nabla}\left(\chi-U^{n}\right)\right)_{\Omega_{f}}+\gamma\left(\mathcal{G}_{f}^{h}\left[\frac{U^{n}-U^{n-1}}{\tau_{n}}\right], \chi-U^{n}\right)_{\Omega_{f}}^{h} \\
& \quad \geq \gamma^{-1}\left(U^{n-1}, \chi-U^{n}\right)_{\Omega_{f}}^{h}+\frac{1}{2} \alpha c_{f}^{\prime}\left(\left|\underline{\nabla} \Phi^{n}\right|^{2}, \chi-U^{n}\right)_{\Omega_{f}} \quad \forall \chi \in K_{f}^{h}(0),
\end{aligned}
$$

and

$$
W^{n}=-\gamma \mathcal{G}_{f}^{h}\left[\frac{U^{n}-U^{n-1}}{\tau_{n}}\right]+\lambda^{n}
$$

where $\lambda^{n} \in \mathbb{R}$. We now seek to show that the function $F: K_{f}^{h}(0) \rightarrow K_{f}^{h}(0)$ is continuous, and then apply the Brouwer fixed point theorem, see e.g. [25, p 358]. It follows from $(3.20 \mathrm{a}, \mathrm{b})$ and $(3.21)$ that a fixed point $U^{n}$ of $F$ corresponds to a solution $\left(\Phi^{n}, U^{n}, W^{n}\right)$ of $(3.1 \mathrm{a}-\mathrm{c})$.

To show that $F: K_{f}^{h}(0) \rightarrow K_{f}^{h}(0)$ is continuous we suppose that there exists a sequence $\left\{U^{(j)}\right\}_{j \geq 0}, U^{(j)} \in K_{f}^{h}(0)$ and $U^{(j)} \rightarrow U \in K_{f}^{h}(0)$ as $j \rightarrow \infty$. It remains to show that $F\left(U^{(j)}\right) \rightarrow F(U) \in K_{f}^{h}(0)$ as $j \rightarrow \infty$. We let $Z^{(j)}=F\left(U^{(j)}\right)$, i.e. on letting $\Phi^{(j)} \in S_{g}^{h}$ be such that

$$
\left(c\left(\underline{x}, U^{(j)}\right) \underline{\nabla} \Phi^{(j)}, \underline{\nabla} \chi\right)=0 \quad \forall \chi \in S_{0}^{h},
$$

we have that $Z^{(j)} \in K_{f}^{h}(0)$ satisfies

$$
\begin{aligned}
& \gamma\left(\underline{\nabla} Z^{(j)}, \underline{\nabla}\left(\chi-Z^{(j)}\right)\right)_{\Omega_{f}}+\gamma\left(\mathcal{G}_{f}^{h}\left[\frac{Z^{(j)}-U^{n-1}}{\tau_{n}}\right], \chi-Z^{(j)}\right)_{\Omega_{f}}^{h} \\
& \quad \geq \gamma^{-1}\left(U^{n-1}, \chi-Z^{(j)}\right)_{\Omega_{f}}^{h}+\frac{1}{2} \alpha c_{f}^{\prime}\left(\left|\underline{\nabla} \Phi^{(j)}\right|^{2}, \chi-Z^{(j)}\right)_{\Omega_{f}} \quad \forall \chi \in K_{f}^{h}(0) .
\end{aligned}
$$

Choosing $\chi=\Phi^{(j)}-x_{1}$ in (3.22a), and noting the Cauchy-Schwarz inequality, and Young's inequality $(2.2)$ we have that

$$
\begin{aligned}
\left(c\left(\underline{x}, U^{(j)}\right),\left|\underline{\nabla}\left(\Phi^{(j)}-x_{1}\right)\right|^{2}\right) & =-\left(c\left(\underline{x}, U^{(j)}\right) \underline{\nabla} x_{1}, \underline{\nabla}\left(\Phi^{(j)}-x_{1}\right)\right) \\
& \leq\left(c\left(\underline{x}, U^{(j)}\right),\left|\underline{\nabla} x_{1}\right|^{2}\right)=\left(c\left(\underline{x}, U^{(j)}\right), 1\right),
\end{aligned}
$$


and so Friedrich's inequality, on recalling (1.6), implies that

$$
\left\|\Phi^{(j)}\right\|_{1} \leq C .
$$

Now choosing $\chi=U^{n-1}$ in (3.22b) and applying (3.12) gives that

$$
\begin{aligned}
& \frac{1}{2}\left\{\gamma\left|Z^{(j)}\right|_{1, \Omega_{f}}^{2}-\gamma^{-1}\left|Z^{(j)}\right|_{h, \Omega_{f}}^{2}\right\}+\gamma \tau_{n}\left(\mathcal{G}_{f}^{h}\left[\frac{Z^{(j)}-U^{n-1}}{\tau_{n}}\right], \frac{Z^{(j)}-U^{n-1}}{\tau_{n}}\right)_{\Omega_{f}}^{h} \\
& \quad \leq \frac{1}{2}\left\{\gamma\left|U^{n-1}\right|_{1, \Omega_{f}}^{2}-\gamma^{-1}\left|U^{n-1}\right|_{h, \Omega_{f}}^{2}\right\}+\frac{1}{2} \alpha c_{f}^{\prime}\left(Z^{(j)}-U^{n-1},\left|\underline{\nabla} \Phi^{(j)}\right|^{2}\right)_{\Omega_{f}} .
\end{aligned}
$$

It follows from (3.24), on noting $U^{n-1}, Z^{(j)} \in K_{f}^{h},(3.17)$ and (3.23), that

$$
\begin{aligned}
\gamma\left|Z^{(j)}\right|_{1, \Omega_{f}}^{2} & \leq \gamma\left|U^{n-1}\right|_{1, \Omega_{f}}^{2}+\gamma^{-1}\left|Z^{(j)}\right|_{h, \Omega_{f}}^{2}+2 \alpha c_{f}^{\prime}\left|\Phi^{(j)}\right|_{1, \Omega_{f}}^{2}-2 \gamma \tau_{n}\left|\mathcal{G}_{f}^{h}\left[\frac{Z^{(j)}-U^{n-1}}{\tau_{n}}\right]\right|_{1, \Omega_{f}}^{2} \\
& \leq C\left(U^{n-1}\right) .
\end{aligned}
$$

Combining (3.25), $Z^{(j)} \in K_{f}^{h}(0)$ and (3.23) yields that

$$
\left\|Z^{(j)}\right\|_{1, \Omega_{f}}^{2}+\left\|\Phi^{(j)}\right\|_{1}^{2} \leq C\left(U^{n-1}\right),
$$

and so we can choose a subsequence $\left\{\left(Z^{\left(j_{p}\right)}, \Phi^{\left(j_{p}\right)}\right)\right\}_{p \geq 0}$ such that

$$
Z^{\left(j_{p}\right)} \rightarrow Z \in K_{f}^{h}(0), \quad \Phi^{\left(j_{p}\right)} \rightarrow \Phi \in S_{g}^{h} \quad \text { as } p \rightarrow \infty .
$$

We note that $c\left(\underline{x}, U^{(j)}\right) \rightarrow c(\underline{x}, U)$ as $j \rightarrow \infty$, due to the linearity of $c(\underline{x}, \cdot)$. Passing to the limit in $(3.22 \mathrm{a}, \mathrm{b})$ with the indexing $(j)$ replaced by $\left(j_{p}\right)$, we obtain that the limiting functions $Z$ and $\Phi$ satisfy

$$
\begin{aligned}
& (c(\underline{x}, U) \underline{\nabla} \Phi, \underline{\nabla} \chi)=0 \quad \forall \chi \in S_{0}^{h}, \\
& \gamma(\underline{\nabla} Z, \underline{\nabla}(\chi-Z))_{\Omega_{f}}+\gamma\left(\mathcal{G}_{f}^{h}\left[\frac{Z-U^{n-1}}{\tau_{n}}\right], \chi-Z\right)_{\Omega_{f}}^{h} \\
& \quad \geq \gamma^{-1}\left(U^{n-1}, \chi-Z\right)_{\Omega_{f}}^{h}+\frac{1}{2} \alpha c_{f}^{\prime}\left(|\underline{\nabla} \Phi|^{2}, \chi-Z\right)_{\Omega_{f}} \quad \forall \chi \in K_{f}^{h}(0),
\end{aligned}
$$

which implies that $Z=F(U)$, and so the limit $Z$ is unique. It follows that the whole sequence converges, i.e. $F\left(U^{(j)}\right)=Z^{(j)} \rightarrow Z=F(U)$ as $j \rightarrow \infty$. Hence $F$ is continuous, and by the Brouwer fixed point theorem we have the existence of a $U \in K_{f}^{h}(0)$ such that $F(U)=U$. On setting $U^{n}=U$, existence of a solution $\left(\Phi^{n}, U^{n}, W^{n}\right)$ to $(3.1 \mathrm{a}-\mathrm{c})$ then follows from (3.1a) and a Friedrich's inequality, and from (3.21).

\subsection{Stability}

We now prove stability for the scheme $(3.1 \mathrm{a}-\mathrm{c})$. 
Theorem 3.5. A solution $\left\{\left(\Phi^{n}, U^{n}, W^{n}\right)\right\}_{n=1}^{N}$ to $(3.1 \mathrm{a}-\mathrm{c})$ with $\Phi^{0} \in S_{g}^{h}$ satisfying (3.2) is such that for $n=1 \rightarrow N$

$$
\begin{aligned}
\mathcal{J}\left(U^{n}, \Phi^{n}\right) & +\sum_{k=1}^{n}\left\{\frac{1}{2} \gamma\left|U^{k}-U^{k-1}\right|_{1, \Omega_{f}}^{2}+\frac{1}{2} \gamma^{-1}\left|U^{k}-U^{k-1}\right|_{h, \Omega_{f}}^{2}\right. \\
& \left.+\frac{1}{2} \alpha\left(c\left(\underline{x}, U^{k-1}\right),\left|\underline{\nabla}\left(\Phi^{k}-\Phi^{k-1}\right)\right|^{2}\right)\right\}+\sum_{k=1}^{n} \frac{\tau_{k}}{\gamma}\left|W^{k}\right|_{1, \Omega_{f}}^{2} \leq \mathcal{J}\left(U^{0}, \Phi^{0}\right) .
\end{aligned}
$$

Proof Combining (3.9) and (3.10) at the time-step $k \geq 1$, leads to the intermediate step

$$
\begin{aligned}
\mathcal{J}\left(U^{k}, \Phi^{k}\right)+ & \frac{1}{2} \gamma\left|\underline{\nabla}\left(U^{k}-U^{k-1}\right)\right|_{0, \Omega_{f}}^{2}+\frac{1}{2} \gamma^{-1}\left|U^{k}-U^{k-1}\right|_{h, \Omega_{f}}^{2}+ \\
& \frac{\tau_{k}}{\gamma}\left|W^{k}\right|_{1, \Omega_{f}}^{2}+\frac{1}{2} \alpha\left(c\left(\underline{x}, U^{k-1}\right),\left|\underline{\nabla}\left(\Phi^{k}-\Phi^{k-1}\right)\right|^{2}\right) \leq \mathcal{J}\left(U^{k-1}, \Phi^{k-1}\right) .
\end{aligned}
$$

Now summing (3.27) over $k=1 \rightarrow n$ gives us the desired result (3.26).

Lemma 3.6. Let $u^{0} \in K_{f} \cap W^{1, s}\left(\Omega_{f}\right)$ for $s>d$, and the assumptions $\left(\mathcal{A}_{1}\right)$ hold. On choosing $U^{0} \equiv \pi_{f}^{h} u^{0}$ it follows that $U^{0} \in K_{f}^{h}$ is such that for all $h>0$,

$$
\left\|U^{0}\right\|_{1, \Omega_{f}} \leq C \text {. }
$$

Proof The result (3.28) follows from (2.18c) since

$$
\left\|\pi_{f}^{h} u^{0}\right\|_{1, \Omega_{f}} \leq C\left\|\pi_{f}^{h} u^{0}\right\|_{1, s, \Omega_{f}} \leq C\left[\left\|\left(I-\pi_{f}^{h}\right) u^{0}\right\|_{1, s, \Omega_{f}}+\left\|u^{0}\right\|_{1, s, \Omega_{f}}\right] \leq\left\|u^{0}\right\|_{1, s, \Omega_{f}} \leq C .
$$

Theorem 3.7. Let $u^{0} \in K_{f} \cap W^{1, s}\left(\Omega_{f}\right)$ for $s>d$ with $f_{\Omega_{f}} u^{0} \mathrm{~d} \underline{x} \in(-1,1)$, and the assumptions $\left(\mathcal{A}_{1}\right)$ hold, and choose $U^{0} \equiv \pi_{f}^{h} u^{0}$. Then for all $h>0$, and for all time partitions $\left\{\tau_{n}\right\}_{n=1}^{N}$, a solution $\left\{\left(\Phi^{n}, U^{n}, W^{n}\right)\right\}_{n=1}^{N}$ to $(3.1 \mathrm{a}-\mathrm{c})$, with $\Phi^{0} \in S_{g}^{h}$ satisfying (3.2), is such that

$$
\max _{n=1 \rightarrow N}\left\|\Phi^{n}\right\|_{1}^{2} \leq C
$$

and

$$
\begin{gathered}
\gamma \max _{n=1 \rightarrow N}\left\|U^{n}\right\|_{1, \Omega_{f}}^{2}+\sum_{n=1}^{N}\left[\gamma\left|U^{n}-U^{n-1}\right|_{1, \Omega_{f}}^{2}+\gamma^{-1}\left|U^{n}-U^{n-1}\right|_{0, \Omega_{f}}^{2}\right] \\
+\sum_{n=1}^{N}\left[\alpha\left|\left[c\left(\underline{x}, U^{n-1}\right)\right]^{\frac{1}{2}} \underline{\nabla}\left(\Phi^{n}-\Phi^{n-1}\right)\right|_{0}^{2}+\gamma^{-1} \tau_{n}\left|W^{n}\right|_{1, \Omega_{f}}^{2}\right] \leq C .
\end{gathered}
$$

Moreover, for h sufficiently small it holds that

$$
\sum_{n=1}^{N} \tau_{n}\left\|W^{n}\right\|_{1, \Omega_{f}}^{2} \leq C
$$

In addition, if $\left(\mathcal{A}_{2}\right)$ holds,

$$
\gamma \sum_{n=1}^{N} \tau_{n}\left|\mathcal{G}_{f}\left[\frac{U^{n}-U^{n-1}}{\tau_{n}}\right]\right|_{1, \Omega_{f}}^{2}+\gamma \tau^{-\frac{1}{2}} \sum_{n=1}^{N}\left|U^{n}-U^{n-1}\right|_{0, \Omega_{f}}^{2} \leq C .
$$


Proof The bound (3.29) follows immediately from (3.15) and (3.7). To obtain the desired result (3.30) we plug (3.8) into (3.26), to obtain for $n=1 \rightarrow N$

$$
\begin{aligned}
& \frac{1}{2}\left\{\gamma\left|U^{n}\right|_{1, \Omega_{f}}^{2}-\gamma^{-1}\left|U^{n}\right|_{h, \Omega_{f}}^{2}\right\}-\frac{1}{2} \alpha\left(c\left(\underline{x}, U^{n}\right),\left|\underline{\nabla} \Phi^{n}\right|^{2}\right) \\
& +\frac{1}{2} \sum_{k=1}^{n}\left[\gamma\left|U^{k}-U^{k-1}\right|_{1, \Omega_{f}}^{2}+\gamma^{-1}\left|U^{k}-U^{k-1}\right|_{h, \Omega_{f}}^{2}\right] \\
& +\sum_{k=1}^{n}\left[\frac{1}{2} \alpha\left|\left[c\left(\underline{x}, U^{k-1}\right)\right]^{\frac{1}{2}} \underline{\nabla}\left(\Phi^{k}-\Phi^{k-1}\right)\right|_{0}^{2}+\gamma^{-1} \tau_{k}\left|W^{k}\right|_{1, \Omega_{f}}^{2}\right] \\
& \leq \frac{1}{2}\left\{\gamma\left|U^{0}\right|_{1, \Omega_{f}}^{2}-\gamma^{-1}\left|U^{0}\right|_{h, \Omega_{f}}^{2}\right\}-\frac{1}{2} \alpha\left(c\left(\underline{x}, U^{0}\right),\left|\underline{\nabla} \Phi^{0}\right|^{2}\right) \leq \frac{1}{2} \gamma\left\|U^{0}\right\|_{1, \Omega_{f}}^{2} .
\end{aligned}
$$

On recalling $(2.17),(2.18 \mathrm{e})$, the fact that $U^{n} \in K_{f}^{h},(3.4)$ and (1.6) we obtain that

$$
\frac{1}{2} \gamma^{-1}\left|U^{n}\right|_{h, \Omega_{f}}^{2}+\frac{1}{2} \alpha\left(c\left(\underline{x}, U^{n}\right),\left|\underline{\nabla} \Phi^{n}\right|^{2}\right) \leq \frac{1}{2} \gamma^{-1}(d+2)\left|\Omega_{f}\right|+\frac{1}{2} \alpha c_{\max }|\Omega| .
$$

Combining (3.33) and (3.34) for $n=1 \rightarrow N$, and noting (3.28), yields the desired result $(3.30)$.

To prove (3.31), we make use of [10, Corollary 3.2]. Choosing $\chi \equiv \pm 1$ in (3.1c), rearranging and noting (3.29), we obtain

$$
\left|\left(W^{n}, 1\right)_{\Omega_{f}}\right| \leq C_{1}+\left(W^{n}, U^{n}\right)_{\Omega_{f}}^{h}+C\left|\underline{\nabla} \Phi^{n}\right|_{0, \Omega_{f}}^{2} \leq C+\left(W^{n}, U^{n}\right)_{\Omega_{f}}^{h},
$$

for $n=1 \rightarrow N$. On recalling (3.3), we set

$$
m_{f}^{h}:=f_{\Omega_{f}} U^{0} \mathrm{~d} \underline{x}=f_{\Omega_{f}} U^{n} \mathrm{~d} \underline{x},
$$

which means that $U^{n}-m_{f}^{h} \in Z_{f}^{h}$. Hence we have that

$$
\begin{aligned}
\left(W^{n}, U^{n}\right)_{\Omega_{f}}^{h} & =\left(W^{n}, U^{n}-m_{f}^{h}\right)_{\Omega_{f}}^{h}+m_{f}^{h}\left(W^{n}, 1\right)_{\Omega_{f}} \\
& =\left(\underline{\nabla} \mathcal{G}_{f}^{h}\left[U^{n}-m_{f}^{h}\right], \underline{\nabla} W^{n}\right)_{\Omega_{f}}+m_{f}^{h}\left(W^{n}, 1\right)_{\Omega_{f}} \\
& \leq\left|\mathcal{G}_{f}^{h}\left[U^{n}-m_{f}^{h}\right]\right|_{1, \Omega_{f}}\left|W^{n}\right|_{1, \Omega_{f}}+m_{f}^{h}\left(W^{n}, 1\right)_{\Omega_{f}} .
\end{aligned}
$$

It follows from $(3.17),(2.18 \mathrm{e})$, the Poincaré inequality and $U^{n} \in K_{f}^{h}$ that

$$
\begin{aligned}
\left|\mathcal{G}_{f}^{h}\left[U^{n}-m_{f}^{h}\right]\right|_{1, \Omega_{f}}^{2} & =\left(U^{n}-m_{f}^{h}, \mathcal{G}_{f}^{h}\left[U^{n}-m_{f}^{h}\right]\right)_{\Omega_{f}}^{h} \leq\left|U^{n}-m_{f}^{h}\right|_{h, \Omega_{f}}\left|\mathcal{G}_{f}^{h}\left[U^{n}-m_{f}^{h}\right]\right|_{h, \Omega_{f}} \\
& \leq C\left|U^{n}-m_{f}^{h}\right|_{h, \Omega_{f}}^{2} \leq C .
\end{aligned}
$$

It follows from (3.36), $U^{0}=\pi_{f}^{h} u^{0}$ and (2.18d) that

$$
m_{f}^{h} \rightarrow f_{\Omega_{f}} u^{0} \mathrm{~d} \underline{x} \in(-1,1) \quad \text { as } h \rightarrow 0,
$$

and so for $h$ sufficiently small, $m_{f}^{h}$ is uniformly bounded away from -1 and 1 , i.e. (1 $\left.\left|m_{f}^{h}\right|\right)^{-1} \leq C$. Hence combining (3.35), (3.37) and (3.38) yields for $h$ sufficiently small that

$$
\left|\left(W^{n}, 1\right)_{\Omega_{f}}\right| \leq C\left(1-\left|m_{f}^{h}\right|\right)^{-1}\left[1+\left|W^{n}\right|_{1, \Omega_{f}}\right] \leq C\left[1+\left|W^{n}\right|_{1, \Omega_{f}}\right], \quad \text { for } n=1 \rightarrow N .
$$


Therefore it follows from the Poincaré inequality and (3.30) that

$$
\sum_{n=1}^{N} \tau_{n}\left\|W^{n}\right\|_{1, \Omega_{f}}^{2} \leq C \sum_{n=1}^{N} \tau_{n}\left[\left|W^{n}\right|_{1, \Omega_{f}}^{2}+\left[\left(W^{n}, 1\right)_{\Omega_{f}}\right]^{2}\right] \leq C \sum_{n=1}^{N} \tau_{n}\left[\left|W^{n}\right|_{1, \Omega_{f}}^{2}+1\right] \leq C,
$$

i.e. the desired result (3.31).

We now prove (3.32). From $(2.5),(2.19),(3.1 \mathrm{~b})$, and $(2.20)$, which requires $\left(\mathcal{A}_{2}\right)$ to hold, we obtain for any $\eta \in H^{1}\left(\Omega_{f}\right)$ that

$$
\begin{aligned}
\gamma\left(\underline{\nabla} \mathcal{G}_{f}\left[\frac{U^{n}-U^{n-1}}{\tau_{n}}\right], \underline{\nabla} \eta\right)_{\Omega_{f}} & =\gamma\left(\frac{U^{n}-U^{n-1}}{\tau_{n}}, \eta\right)_{\Omega_{f}}=\gamma\left(\frac{U^{n}-U^{n-1}}{\tau_{n}}, Q_{f}^{h} \eta\right)_{\Omega_{f}}^{h} \\
& =-\left(\underline{\nabla} W^{n}, \underline{\nabla}\left[Q_{f}^{h} \eta\right]\right)_{\Omega_{f}} \leq C\left|\underline{\nabla} W^{n}\right|_{0, \Omega_{f}}|\eta|_{1, \Omega_{f}},
\end{aligned}
$$

and so

$$
\left|\mathcal{G}_{f}\left[\frac{U^{n}-U^{n-1}}{\tau_{n}}\right]\right|_{1, \Omega_{f}}^{2} \leq \frac{C}{\gamma^{2}}\left|\underline{\nabla} W^{n}\right|_{0, \Omega_{f}}^{2}
$$

The first bound in (3.32) then follows from (3.30).

Moreover we have from (2.5) that

$$
\begin{aligned}
\sum_{n=1}^{N}\left|U^{n}-U^{n-1}\right|_{0, \Omega_{f}}^{2} & =\sum_{n=1}^{N}\left(\underline{\nabla}\left[\mathcal{G}_{f}\left(U^{n}-U^{n-1}\right)\right], \underline{\nabla}\left[U^{n}-U^{n-1}\right]\right)_{\Omega_{f}} \\
& \leq \tau^{\frac{1}{2}}\left[\sum_{n=1}^{N}\left|U^{n}-U^{n-1}\right|_{1, \Omega_{f}}^{2}\right]^{\frac{1}{2}}\left[\sum_{n=1}^{N} \tau_{n}\left|\mathcal{G}_{f}\left[\frac{U^{n}-U^{n-1}}{\tau_{n}}\right]\right|_{1, \Omega_{f}}^{2}\right]^{\frac{1}{2}}
\end{aligned}
$$

The second bound in (3.32) on $\gamma \tau^{-\frac{1}{2}} \sum_{n=1}^{N}\left|U^{n}-U^{n-1}\right|_{0, \Omega_{f}}^{2}$ then follows from (3.39), (3.30), and the first bound in (3.32).

\section{Convergence}

Throughout this section, we restrict ourselves to the case $d=2$. For convenience we rewrite the electro-static potential equation (3.1a). We set

$$
\widetilde{\Phi}^{n}:=\Phi^{n}-x_{1} \in S_{0}^{h},
$$

and from (3.1a) have that

$$
\left(c\left(\underline{x}, U^{n}\right) \underline{\nabla} \widetilde{\Phi}^{n}, \underline{\nabla} \chi\right)=-\left(c\left(\underline{x}, U^{n}\right) \underline{\nabla} x_{1}, \underline{\nabla} \chi\right) \quad \forall \chi \in S_{0}^{h} .
$$

Lemma 4.1. Let $d=2$ and the remaining assumptions of Lemma 2.3 hold. Then for all $p \in\left[2,2+\delta_{1}\right]$ and for all $h \in\left(0, h_{0}\right)$ we have that

$$
\left|\underline{\nabla} \widetilde{\Phi}^{n}\right|_{0, p} \leq C .
$$


Proof It follows from (2.45), (4.1), applying the Hölder inequality, and (1.6) that

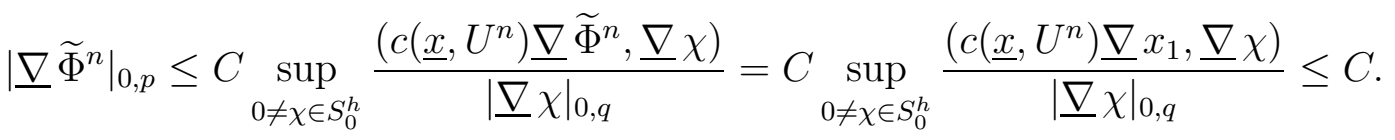

Let

$$
\begin{aligned}
U(t) & :=\frac{t-t_{n-1}}{\tau_{n}} U^{n}+\frac{t_{n}-t}{\tau_{n}} U^{n-1}, \quad t \in\left[t_{n-1}, t_{n}\right], \quad n \geq 1, \\
U^{+}(t) & :=U^{n}, \quad U^{-}(t):=U^{n-1}, \quad t \in\left(t_{n-1}, t_{n}\right], \quad n \geq 1 .
\end{aligned}
$$

Furthermore, let $U^{( \pm)}$denote $U$ with or without the superscript \pm . We note that

$$
U-U^{ \pm}=\left(t-t_{n}^{ \pm}\right) \frac{\partial U}{\partial t}, \quad t \in\left(t_{n-1}, t_{n}\right), \quad n \geq 1,
$$

where $t_{n}^{+}=t_{n}$ and $t_{n}^{-}=t_{n-1}$. We also introduce

$$
\bar{\tau}(t):=\tau_{n}, \quad t \in\left(t_{n-1}, t_{n}\right], \quad n \geq 1 .
$$

We recall the definition of $S_{0}$ from Section 1 , and we define $W_{g}^{1, p}(\Omega):=\left\{\eta \in W^{1, p}(\Omega)\right.$ : $\eta=g^{ \pm}$on $\left.\partial_{D}^{ \pm} \Omega\right\}$, and set $H_{g}^{1}(\Omega):=W_{g}^{1,2}(\Omega)$.

Using the above notation and introducing the analogous notation for $W^{+}$, and $\Phi^{+}$, $(3.1 \mathrm{a}-\mathrm{c})$ can be restated as follows:

Find $\left(\Phi^{+}, U, W^{+}\right) \in L^{\infty}\left(0, T ; S_{g}^{h}\right) \times C\left([0, T] ; K_{f}^{h}\right) \times L^{2}\left(0, T ; S_{f}^{h}\right)$ such that for all $\chi_{0} \in$ $L^{\infty}\left(0, T ; S_{0}^{h}\right), z^{h} \in L^{2}\left(0, T ; K_{f}^{h}\right)$, and $\chi \in L^{2}\left(0, T ; S_{f}^{h}\right)$,

$$
\begin{aligned}
& \int_{0}^{T}\left(c\left(\underline{x}, U^{+}\right) \underline{\nabla} \Phi^{+}, \underline{\nabla} \chi_{0}\right) \mathrm{d} t=0, \\
& \int_{0}^{T}\left[\gamma\left(\frac{\partial U}{\partial t}, \chi\right)_{\Omega_{f}}^{h}+\left(\underline{\nabla} W^{+}, \underline{\nabla} \chi\right)_{\Omega_{f}}\right] \mathrm{d} t=0, \\
& \gamma \int_{0}^{T}\left(\underline{\nabla} U^{+}, \underline{\nabla}\left(z^{h}-U^{+}\right)\right)_{\Omega_{f}} \mathrm{~d} t \geq \int_{0}^{T}\left(W^{+}+\gamma^{-1} U^{-}, z^{h}-U^{+}\right)_{\Omega_{f}}^{h} \mathrm{~d} t \\
& \quad+\frac{1}{2} \alpha c_{f}^{\prime} \int_{0}^{T}\left(\left|\underline{\nabla} \Phi^{+}\right|^{2}, z^{h}-U^{+}\right)_{\Omega_{f}} \mathrm{~d} t .
\end{aligned}
$$

Lemma 4.2. Let $d=2$ and all the assumptions of Theorem 3.7 hold. Then there exists a subsequence of $\left\{\left(\Phi^{+}, U, W^{+}\right)\right\}_{h}$, where $\left(\Phi^{+}, U, W^{+}\right)$solve $(3.1 \mathrm{a}-\mathrm{c})$, and functions

$$
u \in L^{\infty}\left(0, T ; K_{f}\right) \cap H^{1}\left(0, T ;\left(H^{1}\left(\Omega_{f}\right)\right)^{\prime}\right) \quad \text { and } \quad w \in L^{2}\left(0, T ; H^{1}\left(\Omega_{f}\right)\right),
$$

with $u(\cdot, 0)=u^{0}$ and $f_{\Omega_{f}} u(\underline{x}, t) \mathrm{d} \underline{x}=f_{\Omega_{f}} u^{0}(\underline{x}) \mathrm{d} \underline{x}$ for a.a. $t \in(0, T)$ such that as $h, \tau \rightarrow 0$

$U, U^{ \pm} \rightarrow u$

$\mathcal{G}_{f} \frac{\partial U}{\partial t} \rightarrow \mathcal{G}_{f} \frac{\partial u}{\partial t}$

$U, U^{ \pm} \rightarrow u$

$W^{+} \rightarrow w$ weak- * in $L^{\infty}\left(0, T ; H^{1}\left(\Omega_{f}\right)\right)$,

weakly in $L^{2}\left(0, T ; H^{1}\left(\Omega_{f}\right)\right)$,

strongly in $L^{2}\left(0, T ; L^{s}\left(\Omega_{f}\right)\right)$,

weakly in $L^{2}\left(0, T ; H^{1}\left(\Omega_{f}\right)\right)$, 
for all $s \in[2, \infty)$. Additionally, there exists a function

$$
\phi \in L^{\infty}\left(0, T ; W_{g}^{1,2+\delta_{1}}(\Omega)\right),
$$

where $\delta_{1}>0$ is as in Lemmas 2.3 and 4.1, such that as $h, \tau \rightarrow 0$ the subsequence above satisfies

$$
\begin{array}{ll}
\Phi^{+} \rightarrow \phi & \text { weak-* in } L^{\infty}\left(0, T ; W_{g}^{1,2+\delta_{1}}(\Omega)\right), \\
\Phi^{+} \rightarrow \phi & \text { strongly in } L^{2}\left(0, T ; S_{g}\right) .
\end{array}
$$

Moreover, $(\phi, u)$ satisfy

$$
\int_{0}^{T}(c(\underline{x}, u) \underline{\nabla} \phi, \underline{\nabla} \eta) \mathrm{d} t=0 \quad \forall \eta \in L^{2}\left(0, T ; S_{0}\right) .
$$

Proof On noting the definitions (4.3a,b), (4.5), the bounds (3.30), (3.31) and (3.32) imply that

$$
\begin{aligned}
\left\|U^{( \pm)}\right\|_{L^{\infty}\left(0, T ; H^{1}\left(\Omega_{f}\right)\right)}^{2}+\left\|\bar{\tau}^{\frac{1}{2}} \frac{\partial U}{\partial t}\right\|_{L^{2}\left(0, T ; H^{1}\left(\Omega_{f}\right)\right)}^{2}+\left\|W^{+}\right\|_{L^{2}\left(0, T ; H^{1}\left(\Omega_{f}\right)\right)}^{2} & \\
& +\left\|\mathcal{G}_{f} \frac{\partial U}{\partial t}\right\|_{L^{2}\left(0, T ; H^{1}\left(\Omega_{f}\right)\right)}^{2}+\tau^{-\frac{1}{2}}\left\|\bar{\tau}^{\frac{1}{2}} \frac{\partial U}{\partial t}\right\|_{L^{2}\left(0, T ; L^{2}\left(\Omega_{f}\right)\right)}^{2} \leq C .
\end{aligned}
$$

Furthermore, we deduce from (4.4) and (4.12) that

$$
\left\|U-U^{ \pm}\right\|_{L^{2}\left(0, T ; H^{1}\left(\Omega_{f}\right)\right)}^{2} \leq\left\|\bar{\tau} \frac{\partial U}{\partial t}\right\|_{L^{2}\left(0, T ; H^{1}\left(\Omega_{f}\right)\right)}^{2} \leq C \tau .
$$

The bound on $\left\|U^{( \pm)}\right\|_{L^{\infty}\left(0, T ; H^{1}\left(\Omega_{f}\right)\right)}^{2}$ from (4.12) means that $\left\{U^{( \pm)}\right\}_{h}$ forms a closed and bounded set in $L^{\infty}\left(0, T ; H^{1}\left(\Omega_{f}\right)\right)$. Therefore we can choose a subsequence of $\left\{U^{( \pm)}\right\}_{h}$ such that, as $h, \tau \rightarrow 0$

$$
U^{( \pm)} \rightarrow u^{( \pm)}, \quad \text { weak- }{ }^{*} \text { in } L^{\infty}\left(0, T ; H^{1}\left(\Omega_{f}\right)\right) .
$$

Now (4.13) implies that $u^{+}=u^{-}=u$, which yields (4.8a). The limit satisfies $u \in$ $L^{\infty}\left(0, T ; H^{1}\left(\Omega_{f}\right)\right)$, but this can be strengthened to $u \in L^{\infty}\left(0, T ; K_{f}\right)$, since $U, U^{ \pm} \in$ $L^{\infty}\left(0, T ; K_{f}\right)$, and $K_{f} \subset H^{1}\left(\Omega_{f}\right)$ is closed and convex.

Furthermore, the bound on $\left\|\mathcal{G}_{f} \frac{\partial U}{\partial t}\right\|_{L^{2}\left(0, T ; H^{1}\left(\Omega_{f}\right)\right)}^{2}$ in (4.12) implies, on extracting a further subsequence, that as $h, \tau \rightarrow 0$

$$
\mathcal{G}_{f} \frac{\partial U}{\partial t} \rightarrow z \quad \text { weakly in } L^{2}\left(0, T ; H^{1}\left(\Omega_{f}\right)\right) .
$$

It remains to show that $z=\mathcal{G}_{f} u_{t}$. From (2.5), and (4.14) we have for $\eta \in L^{2}\left(0, T ; H^{1}\left(\Omega_{f}\right)\right.$ ), that as $h, \tau \rightarrow 0$

$$
\int_{0}^{T}\left(U_{t}, \eta\right)_{\Omega_{f}} \mathrm{~d} t=\int_{0}^{T}\left(\underline{\nabla} \mathcal{G}_{f} U_{t}, \underline{\nabla} \eta\right)_{\Omega_{f}} \mathrm{~d} t \rightarrow \int_{0}^{T}(\underline{\nabla} z, \underline{\nabla} \eta)_{\Omega_{f}} \mathrm{~d} t .
$$

Suppose we take $\eta \in H^{1}\left(0, T ; H^{1}\left(\Omega_{f}\right)\right)$ such that $\eta(0)=\eta(T)=0$. Now using integration by parts, and (4.8a) we have that as $h, \tau \rightarrow 0$

$$
\int_{0}^{T}\left(U_{t}, \eta\right)_{\Omega_{f}} \mathrm{~d} t=-\int_{0}^{T}\left(U, \eta_{t}\right)_{\Omega_{f}} \mathrm{~d} t \rightarrow-\int_{0}^{T}\left(u, \eta_{t}\right)_{\Omega_{f}} \mathrm{~d} t=\int_{0}^{T}\left\langle u_{t}, \eta\right\rangle_{\Omega_{f}} \mathrm{~d} t .
$$


Combining (4.15) and (4.16) gives us the results (4.7) for $u$, and (4.8b).

On recalling that the embedding $H^{1}\left(\Omega_{f}\right) \hookrightarrow L^{s}\left(\Omega_{f}\right)$ is compact by the Sobolev embedding theorem for $d=2$, it follows from (2.4a) and the bounds on $\|U\|_{L^{\infty}\left(0, T ; H^{1}\left(\Omega_{f}\right)\right)}^{2}$ and $\left\|\mathcal{G}_{f} \frac{\partial U}{\partial t}\right\|_{L^{2}\left(0, T ; H^{1}\left(\Omega_{f}\right)\right)}^{2}$ from (4.12) that as $h, \tau \rightarrow 0$

$$
U \rightarrow u \quad \text { strongly in } L^{2}\left(0, T ; L^{s}\left(\Omega_{f}\right)\right) .
$$

Combining (4.17) and (4.13) yields that (4.8c) holds. Noting the bound on $W^{+}$in (4.12), and on extracting a possible further subsequence yields that (4.7) for $w$ and (4.8d) hold.

Then (4.7) for $u$, our assumptions on $u^{0}$, (3.3) yield, on noting the embedding result (2.4b), $U^{0}=\pi^{h} u^{0}$ and (2.18c), that the subsequence satisfies the additional initial and integral conditions.

We now prove the results (4.9), (4.10a,b) and (4.11). From (3.5) and (4.2) we have for $d=2$ that

$$
\left\|\Phi^{+}\right\|_{L^{\infty}\left(0, T ; W_{g}^{1,2+\delta_{1}}(\Omega)\right)}^{2} \leq C .
$$

Hence, on noting $\Phi^{+}(\cdot, t) \in S_{g}^{h}$, we can take a further subsequence of $\left\{\left(\Phi^{+}, U, W^{+}\right)\right\}_{h}$ such that (4.9) and (4.10a) hold.

For any $\eta \in C\left([0, T] ; S_{0} \cap H^{2}(\Omega)\right)$, we choose $\chi_{0} \equiv \pi^{h} \eta$ in (4.6a). The desired result (4.11) then follows from $(2.18 \mathrm{~b}),(4.18),(1.5 \mathrm{a}),(4.8 \mathrm{c}),(4.10 \mathrm{a})$ and a density result. We have from (4.6a) and (4.11) that

$$
\begin{aligned}
& \int_{0}^{T}\left(c(\underline{x}, u) \underline{\nabla}\left(\phi-\Phi^{+}\right), \underline{\nabla}\left(\phi-\Phi^{+}\right)\right) \mathrm{d} t=\int_{0}^{T}\left(c(\underline{x}, u) \underline{\nabla} \Phi^{+}, \underline{\nabla}\left(\Phi^{+}-\phi\right)\right) \mathrm{d} t \\
& =\int_{0}^{T}\left(c(\underline{x}, u) \underline{\nabla} \Phi^{+}, \underline{\nabla}\left(\pi^{h} \phi-\phi\right)\right) \mathrm{d} t+\int_{0}^{T}\left(\left[c(\underline{x}, u)-c\left(\underline{x}, U^{+}\right)\right] \underline{\nabla} \Phi^{+}, \underline{\nabla}\left(\Phi^{+}-\pi^{h} \phi\right)\right) \mathrm{d} t .
\end{aligned}
$$

The desired result (4.10b) then follows from (4.19) on noting (1.5a), (2.18d), (4.9), (4.18), and $(4.8 \mathrm{c})$.

Theorem 4.3. Let $d=2$ and the assumptions of Lemma 4.2 hold. Then there exists a subsequence of $\left\{\left(\Phi^{+}, U, W^{+}\right)\right\}_{h}$, where $\left(\Phi^{+}, U, W^{+}\right)$solve $(3.1 \mathrm{a}-\mathrm{c})$, and functions $(\phi, u, w)$ satisfying (4.7) and (4.9). In addition, as $h, \tau \rightarrow 0$ the following hold: (4.8a-d) and (4.10a,b) for almost all $t \in(0, T)$. Furthermore, we have that $(\phi, u, w)$ fulfill $u(\cdot, 0)=u^{0}$ and $f_{\Omega_{f}} u(\underline{x}, t) \mathrm{d} \underline{x}=f_{\Omega_{f}} u^{0}(\underline{x}) \mathrm{d} \underline{x}$ for almost all $t \in(0, T)$, and satisfy (4.11) and

$$
\begin{gathered}
\gamma \int_{0}^{T}\left\langle\frac{\partial u}{\partial t}, \eta\right\rangle_{H^{1}\left(\Omega_{f}\right)} \mathrm{d} t+\int_{0}^{T}(\underline{\nabla} w, \underline{\nabla} \eta)_{\Omega_{f}} \mathrm{~d} t=0 \quad \forall \eta \in L^{2}\left(0, T ; H^{1}\left(\Omega_{f}\right)\right), \\
\gamma \int_{0}^{T}(\underline{\nabla} u, \underline{\nabla}(\eta-u))_{\Omega_{f}} \mathrm{~d} t-\int_{0}^{T}\left(w+\gamma^{-1} u, \eta-u\right)_{\Omega_{f}} \mathrm{~d} t \\
-\frac{1}{2} \alpha c_{f}^{\prime} \int_{0}^{T}\left(|\underline{\nabla} \phi|^{2}, \eta-u\right)_{\Omega_{f}} \mathrm{~d} t \geq 0 \quad \forall \eta \in L^{2}\left(0, T ; K_{f}\right) .
\end{gathered}
$$


Proof For any $\eta \in H^{1}\left(0, T ; H^{2}\left(\Omega_{f}\right)\right)$ we choose $\chi \equiv \pi^{h} \eta$ in (4.6b) and now analyse the subsequent terms. First, (2.18f), the embedding $H^{1}(0, T ; X) \hookrightarrow C([0, T] ; X),(4.12)$, and (2.18b) yield that

$$
\begin{aligned}
& \left|\int_{0}^{T}\left[\left(\frac{\partial U}{\partial t}, \pi_{f}^{h} \eta\right)_{\Omega_{f}}^{h}-\left(\frac{\partial U}{\partial t}, \pi_{f}^{h} \eta\right)_{\Omega_{f}}\right] \mathrm{d} t\right| \\
& =\mid-\int_{0}^{T}\left(U, \frac{\partial\left(\pi_{f}^{h} \eta\right)}{\partial t}\right)_{\Omega_{f}}^{h} \mathrm{~d} t+\left(U(\cdot, T), \pi_{f}^{h} \eta(\cdot, T)\right)_{\Omega_{f}}^{h}-\left(U(\cdot, 0), \pi_{f}^{h} \eta(\cdot, 0)\right)_{\Omega_{f}}^{h} \\
& \quad+\int_{0}^{T}\left(U, \frac{\partial\left(\pi_{f}^{h} \eta\right)}{\partial t}\right)_{\Omega_{f}} \mathrm{~d} t+\left(U(\cdot, T), \pi_{f}^{h} \eta(\cdot, T)\right)_{\Omega_{f}}-\left(U(\cdot, 0), \pi_{f}^{h} \eta(\cdot, 0)\right)_{\Omega_{f}} \mid \\
& \leq \int_{0}^{T}\left|\left(U, \frac{\partial\left(\pi_{f}^{h} \eta\right)}{\partial t}\right)_{\Omega_{f}}-\left(U, \frac{\partial\left(\pi_{f}^{h} \eta\right)}{\partial t}\right)_{\Omega_{f}}^{h}\right| \mathrm{d} t+\left|\left(U(\cdot, T), \pi_{f}^{h} \eta(\cdot, T)\right)_{\Omega_{f}}^{h}-\left(U(\cdot, T), \pi_{f}^{h} \eta(\cdot, T)\right)_{\Omega_{f}}\right| \\
& \quad \quad+\left|\left(U(\cdot, 0), \pi_{f}^{h} \eta(\cdot, 0)\right)_{\Omega_{f}}-\left(U(\cdot, 0), \pi_{f}^{h} \eta(\cdot, 0)\right)_{\Omega_{f}}^{h}\right| \\
& \leq \int_{0}^{T} C h|U(\cdot, t)|_{0, \Omega_{f}}\left|\frac{\partial\left(\pi_{f}^{h} \eta\right)}{\partial t}\right|_{1} \mathrm{~d} t+C h|U(\cdot, T)|_{0, \Omega_{f}}\left|\pi_{f}^{h} \eta(\cdot, T)\right|_{1, \Omega_{f}} \\
& \quad+C h|U(\cdot, 0)|_{0, \Omega_{f}}\left|\pi_{f}^{h} \eta(\cdot, 0)\right|_{1, \Omega_{f}} \\
& \leq C h\|U\|_{L^{\infty}\left(0, T ; H^{1}\left(\Omega_{f}\right)\right)}\left\|\pi_{f}^{h} \eta\right\|_{H^{1}\left(0, T ; H^{1}\left(\Omega_{f}\right)\right)} \leq C h\|\eta\|_{H^{1}\left(0, T ; H^{2}\left(\Omega_{f}\right)\right) .}
\end{aligned}
$$

Furthermore, it follows from (2.5), (4.12), (2.18b) that

$$
\begin{aligned}
\left|\int_{0}^{T}\left(\frac{\partial U}{\partial t},\left(I-\pi_{f}^{h}\right) \eta\right)_{\Omega_{f}} \mathrm{~d} t\right| & =\left|\int_{0}^{T}\left(\underline{\nabla}\left(\mathcal{G}_{f} \frac{\partial U}{\partial t}\right), \underline{\nabla}\left(\left(I-\pi_{f}^{h}\right) \eta\right)\right)_{\Omega_{f}} \mathrm{~d} t\right| \\
& \leq C\left\|\mathcal{G}_{f} \frac{\partial U}{\partial t}\right\|_{L^{2}\left(0, T ; H^{1}\left(\Omega_{f}\right)\right)}\left\|\left(\pi_{f}^{h}-I\right) \eta\right\|_{L^{2}\left(0, T ; H^{1}\left(\Omega_{f}\right)\right)} \\
& \leq C h\|\eta\|_{L^{2}\left(0, T ; H^{2}\left(\Omega_{f}\right)\right) .}
\end{aligned}
$$

Combining (4.21), (4.22), (4.8b) and (2.5) yields that

$$
\begin{aligned}
\int_{0}^{T}\left(\frac{\partial U}{\partial t}, \pi_{f}^{h} \eta\right)_{\Omega_{f}}^{h} \mathrm{~d} t= & \int_{0}^{T}\left[\left(\frac{\partial U}{\partial t}, \pi_{f}^{h} \eta\right)_{\Omega_{f}}^{h}-\left(\frac{\partial U}{\partial t}, \pi_{f}^{h} \eta\right)_{\Omega_{f}}\right] \mathrm{d} t \\
& +\int_{0}^{T}\left(\frac{\partial U}{\partial t},\left(\pi_{f}^{h}-I\right) \eta\right)_{\Omega_{f}} \mathrm{~d} t+\int_{0}^{T}\left(\frac{\partial U}{\partial t}, \eta\right)_{\Omega_{f}} \mathrm{~d} t \\
\rightarrow & \int_{0}^{T}\left\langle\frac{\partial u}{\partial t}, \eta\right\rangle_{H^{1}\left(\Omega_{f}\right)} \mathrm{d} t \quad \text { as } h, \tau \rightarrow 0 .
\end{aligned}
$$

In view of $(4.12)$, and $(2.18 \mathrm{~b})$ we deduce that

$$
\begin{aligned}
& \left|\int_{0}^{T}\left(\underline{\nabla} W^{+}, \underline{\nabla}\left(I-\pi_{f}^{h}\right) \eta\right)_{\Omega_{f}} \mathrm{~d} t\right| \leq\left.\left|\int_{0}^{T}\right| \underline{\nabla} W^{+}\right|_{0, \Omega_{f}}\left|\left(I-\pi_{f}^{h}\right) \eta\right|_{1, \Omega_{f}} \mathrm{~d} t \mid \\
& \leq\left\|\underline{\nabla} W^{+}\right\|_{L^{2}\left(0, T ; L^{2}\left(\Omega_{f}\right)\right.}\left\|\left(I-\pi_{f}^{h}\right) \eta\right\|_{L^{2}\left(0, T ; H^{1}\left(\Omega_{f}\right)\right)} \leq C h\|\eta\|_{L^{2}\left(0, T ; H^{2}\left(\Omega_{f}\right)\right)} .
\end{aligned}
$$

It follows from (4.24), and (4.8d) that as $h, \tau \rightarrow 0$

$$
\int_{0}^{T}\left(\underline{\nabla} W^{+}, \underline{\nabla} \pi_{f}^{h} \eta\right)_{\Omega_{f}} \mathrm{~d} t \rightarrow \int_{0}^{T}(\underline{\nabla} w, \underline{\nabla} \eta) \mathrm{d} t .
$$


Combining $(4.6 \mathrm{~b}),(4.23),(4.25)$, and the denseness of $H^{1}\left(0, T ; H^{2}\left(\Omega_{f}\right)\right)$ in $L^{2}\left(0, T ; H^{1}\left(\Omega_{f}\right)\right)$ yields the desired result (4.20a), on recalling (4.7).

We now prove $(4.20 \mathrm{~b})$. For any $\eta \in L^{2}\left(0, T ; K_{f} \cap C^{\infty}\left(\overline{\Omega_{f}}\right)\right)$ we choose $z^{h} \equiv \pi_{f}^{h} \eta \in K_{f}^{h}$ in $(4.6 \mathrm{c})$ and obtain

$$
\begin{aligned}
\gamma \int_{0}^{T}\left(\underline{\nabla} U^{+}, \underline{\nabla}\left(\pi_{f}^{h} \eta-U^{+}\right)\right)_{\Omega_{f}} \mathrm{~d} t \geq \int_{0}^{T} & \left(W^{+}+\gamma^{-1} U^{-}, \pi_{f}^{h} \eta-U^{+}\right)_{\Omega_{f}}^{h} \mathrm{~d} t \\
& +\frac{1}{2} \alpha c_{f}^{\prime} \int_{0}^{T}\left(\left|\underline{\nabla} \Phi^{+}\right|^{2}, \pi_{f}^{h} \eta-U^{+}\right)_{\Omega_{f}} \mathrm{~d} t .
\end{aligned}
$$

Looking at each term individually, we first note from (4.8a) and (2.18b) that as $h, \tau \rightarrow 0$

$$
\int_{0}^{T}\left(\underline{\nabla} U^{+}, \underline{\nabla} \pi_{f}^{h} \eta\right)_{\Omega_{f}} \mathrm{~d} t \rightarrow \int_{0}^{T}(\underline{\nabla} u, \underline{\nabla} \eta)_{\Omega_{f}} \mathrm{~d} t
$$

Similarly, by (3.12) and (4.8a) we have that as $h, \tau \rightarrow 0$

$$
\begin{aligned}
\int_{0}^{T} & \left(\underline{\nabla} U^{+}, \underline{\nabla} U^{+}\right)_{\Omega_{f}} \mathrm{~d} t \\
& =\int_{0}^{T}(\underline{\nabla} u, \underline{\nabla} u)_{\Omega_{f}} \mathrm{~d} t+\int_{0}^{T}\left|\underline{\nabla}\left(u-U^{+}\right)\right|_{0, \Omega_{f}}^{2} \mathrm{~d} t-2 \int_{0}^{T}\left(\underline{\nabla} u, \underline{\nabla}\left(u-U^{+}\right)\right)_{\Omega_{f}} \mathrm{~d} t \\
& \geq \int_{0}^{T}(\underline{\nabla} u, \underline{\nabla} u)_{\Omega_{f}} \mathrm{~d} t .
\end{aligned}
$$

By (2.18f), (2.18b) and (4.12) we have that

$$
\begin{aligned}
& \left|\int_{0}^{T}\left[\left(W^{+}+\gamma^{-1} U^{-}, \pi_{f}^{h} \eta-U^{+}\right)_{\Omega_{f}}-\left(W^{+}+\gamma^{-1} U^{-}, \pi_{f}^{h} \eta-U^{+}\right)_{\Omega_{f}}^{h}\right] \mathrm{d} t\right| \\
& \quad \leq C h\left(\left(\int_{0}^{T}\left|W^{+}\right|_{0, \Omega_{f}}^{2} \mathrm{~d} t\right)^{\frac{1}{2}}+1\right)\left(\int_{0}^{T}\left|\pi_{f}^{h} \eta\right|_{1, \Omega_{f}}^{2} \mathrm{~d} t+\int_{0}^{T}\left|U^{+}\right|_{1, \Omega_{f}}^{2} \mathrm{~d} t\right) \leq C h .
\end{aligned}
$$

Furthermore, by (4.8d), (4.8c), and (2.18b) we have that as $h, \tau \rightarrow 0$

$$
\int_{0}^{T}\left(W^{+}+\gamma^{-1} U^{-}, \pi_{f}^{h} \eta-U^{+}\right)_{\Omega_{f}} \mathrm{~d} t \rightarrow \int_{0}^{T}\left(w+\gamma^{-1} u, \eta-u\right)_{\Omega_{f}} \mathrm{~d} t .
$$

Moreover, by (1.5a), (4.10a), (4.10b), (2.18b), (4.8c) that

$$
c_{f}^{\prime} \int_{0}^{T}\left(\left|\underline{\nabla} \Phi^{+}\right|^{2}, \pi_{f}^{h} \eta-U^{+}\right)_{\Omega_{f}} \mathrm{~d} t \rightarrow c_{f}^{\prime} \int_{0}^{T}\left(|\underline{\nabla} \phi|^{2}, \eta-u\right)_{\Omega_{f}} \mathrm{~d} t \quad \text { as } h, \tau \rightarrow 0 .
$$

Combining (4.26)-(4.31), we have that $(\phi, u, w)$ satisfy

$$
\begin{gathered}
\gamma \int_{0}^{T}(\underline{\nabla} u, \underline{\nabla}(\eta-u))_{\Omega_{f}} \mathrm{~d} t-\int_{0}^{T}\left(w+\gamma^{-1} u, \eta-u\right)_{\Omega_{f}}-\frac{1}{2} \alpha c_{f}^{\prime} \int_{0}^{T}\left(|\underline{\nabla} \phi|^{2}, \eta-u\right)_{\Omega_{f}} \mathrm{~d} t \\
\geq 0 \quad \forall \eta \in L^{2}\left(0, T ; K_{f} \cap C^{\infty}\left(\overline{\Omega_{f}}\right)\right) .
\end{gathered}
$$

The desired result (4.20b) now follows from the fact that $K_{f} \cap C^{\infty}\left(\overline{\Omega_{f}}\right)$ is dense in $K_{f}$. 


\section{$5 \quad$ Numerical results}

In this section we present some numerical results for our finite element approximations $(3.1 \mathrm{a}-\mathrm{c})$.

We fix our domain to be $\Omega=(-1.25,1) \times(-1,1)^{d-1}$ throughout, where $\Omega_{f}=(-1,1)^{d}$ and $\Omega_{s}=(-1.25,-1) \times(-1,1)^{d-1}$, i.e. $L_{i}=1, i=1 \rightarrow d$, and $a=0.25$. Throughout this section, unless stated otherwise, we fix the parameters $c_{0}=1, c_{1}=1.25, \gamma=\frac{1}{8 \pi}, \alpha=$ $100, \tau_{n}=\tau=10^{-6}, n=1 \rightarrow N$.

For the initial conditions $u^{0}(\underline{x})$ we take some perturbation from the uniform interface, a straight line in two dimensions, or a plane in three dimensions. If the electric field is removed from the system (by setting $\alpha=0$ ) these perturbations would decay to zero. In particular, we use the smooth non-symmetric quasi-random initial conditions given by

$$
u^{0}(\underline{x}):= \begin{cases}-1 & r(\underline{x}) \leq-\frac{\gamma \pi}{2} \\ 1 & r(\underline{x}) \geq \frac{\gamma \pi}{2} \\ \sin \frac{r(\underline{x})}{\gamma} & |r(\underline{x})|<\frac{\gamma \pi}{2}\end{cases}
$$

where, for $d=2, r(\underline{x}):=-x_{1}-\frac{1}{2}+\sum_{i=1}^{10} \epsilon_{i} \cos \left(\frac{n_{i} \pi\left(x_{2}-y_{i}\right)}{L_{2}}\right) n_{i} \in[-15,15], \epsilon_{i} \in\left(0, \frac{1}{60}\right)$, and $y_{i} \in[0,1)$. For $d=3, r(\underline{x}):=-x_{1}-\frac{1}{2}+\sum_{i=1}^{k} \epsilon_{i} \cos \left(\frac{n_{i} \pi\left(x_{2}-y_{i}\right)}{L_{2}}\right) \cos \left(\frac{\tilde{n}_{i} \pi\left(x_{3}-\tilde{y}_{i}\right)}{L_{3}}\right)$ with $n_{i}, \tilde{n}_{i} \in[-25,25], \epsilon_{i} \in\left(0, \frac{1}{20}\right)$, and $y_{i}, \tilde{y}_{i} \in[0,1)$. Hence (5.1) represents a perturbed straight interface at $x_{1} \approx-\frac{1}{2}$ within the domain $\Omega_{f}$.

\subsection{Solution Methods}

We implemented our finite element approximation within the framework of the finite element code Alberta-3.0-rc6, a release candidate for a successor of Alberta 2.0.1, see [27]. The code uses bisectioning, and its reversal, for refining and coarsening, respectively. Our mesh refinement strategy is described in detail in [29]. It results in a fine mesh of mesh size $h_{f}:=\frac{2}{N_{f}}$ in the interfacial region, and in a coarser mesh of mesh size $h_{c}:=\frac{2}{N_{c}}$ away from it. Here $N_{c}<N_{f}$ are two given parameters, and unless otherwise stated we use $N_{f}=128$ and $N_{c}=16$ throughout this section.

For the solution of the system $(3.1 \mathrm{a}-\mathrm{c})$ we employ the fixed point iteration $(3.16 \mathrm{a}-\mathrm{c})$. We note that (3.16a) is independent of the $\left(U^{n, i}, W^{n, i}\right)$ system, so we solve it first to obtain $\Phi^{n, i}$. Here we stress that it is very impractical to use two separate, overlapping meshes for $S^{h}$ and $S_{f}^{h}$. On recalling assumption $\left(\mathcal{A}_{2}\right)$, we use the fact that $S^{h}$ can be represented as

$$
\begin{gathered}
S^{h}=\left\{\left(\chi_{s}, \chi_{f}\right) \in C\left(\bar{\Omega}_{s}\right) \times C\left(\bar{\Omega}_{f}\right):\left.\chi_{s}\right|_{\sigma} \text { is linear } \forall \sigma \in \mathcal{T}_{s}^{h},\left.\quad \chi_{f}\right|_{\sigma} \text { is linear } \forall \sigma \in \mathcal{T}_{f}^{h},\right. \\
\left.\chi_{s}=\chi_{f} \text { on } \bar{\Omega}_{s} \cap \bar{\Omega}_{f}\right\} .
\end{gathered}
$$


Hence in practice we triangulate $\bar{\Omega}_{s}$ and $\bar{\Omega}_{f}$ separately, and we formulate (3.16a) for $\Phi^{n, i}=\left(\Phi_{s}, \Phi_{f}\right) \in S_{s}^{h} \times S_{f}^{h}$, where $S_{s}^{h}$ is defined similarly to $S_{f}^{h}$. Note that for simplicity we ignore the Dirichlet conditions on $\Phi^{n, i}$ for this discussion. Following a similar strategy used in [5,6], we define the orthogonal projection $\mathcal{P}: S_{s}^{h} \times S_{f}^{h} \rightarrow S^{h}$, and note that the linear equations arising from (3.16a) can now be formulated as

$$
\mathcal{P}\left(\begin{array}{cc}
A_{s} & 0 \\
0 & A_{f}
\end{array}\right) \mathcal{P}\left(\begin{array}{l}
\Phi_{s} \\
\Phi_{f}
\end{array}\right)=0,
$$

where

$$
\begin{array}{ll}
{\left[A_{s}\right]_{j k}=\left(c(\underline{x}, 1) \underline{\nabla} \chi_{j}, \underline{\nabla} \chi_{k}\right)} & \forall j, k \in J_{s}, \\
{\left[A_{f}\right]_{j k}=\left(c\left(\underline{x}, U^{n, i-1}\right) \underline{\nabla} \chi_{j}, \underline{\nabla} \chi_{k}\right)} & \forall j, k \in J_{f} .
\end{array}
$$

The system (5.2), which is symmetric and positive semidefinite, can be solved with a conjugate gradient iterative solver.

Having obtained $\Phi^{n, i}=\left(\Phi_{s}, \Phi_{f}\right)$, we solve the system $(3.16 \mathrm{~b}, \mathrm{c})$ for $\left(U^{n, i}, W^{n, i}\right)$, see [29] for details. Here we stress that for the definition of the right hand side in (3.16c) only $\Phi_{f}$ is needed, and that thanks to our formulation above no transfer of information from one mesh to the other is necessary. This is the main advantage of not using two overlapping meshes for $S^{h}$ and $S_{f}^{h}$.

We repeat the iteration $(3.16 \mathrm{a}-\mathrm{c})$ until

$$
\left|U^{n, i}-U^{n, i-1}\right|_{\infty}+\left|\Phi^{n, i}-\Phi^{n, i-1}\right|_{\infty}<10^{-7} .
$$

\subsection{Numerical results in $2 \mathrm{~d}$}

Figure 2 demonstrates the evolving morphology of our solution. The evolution shows that initial perturbations in a uniform interface coagulate into fewer larger pillar structures, as in Figure 2b. As the morphology progresses, the pillar structures absorb more of the intermediate phase material and grow in size, extending out into the film region, see Figures 2c and 2d.

The underlying mesh for the electric field at the final time-step is displayed in Figure 3, demonstrating the alignment of the meshes across the substrate and film boundary. We can see clearly how the adaptive meshes are tracking the evolution of the interface, and how the meshes for $\Omega_{s}$ and $\Omega_{f}$ are aligning without hanging nodes.

A plot of $\mathcal{J}\left(U^{n}, \Phi^{n}\right)$ over time is shown in Figure 4, where, as expected, we observe that the discrete energy is monotonically decreasing.

\subsection{Numerical results in $3 \mathrm{~d}$}

Figure 5 demonstrates the evolving morphology of our solution, whilst Figure 6 demonstrates the underlying mesh. We can see clearly how the adaptive meshes are 


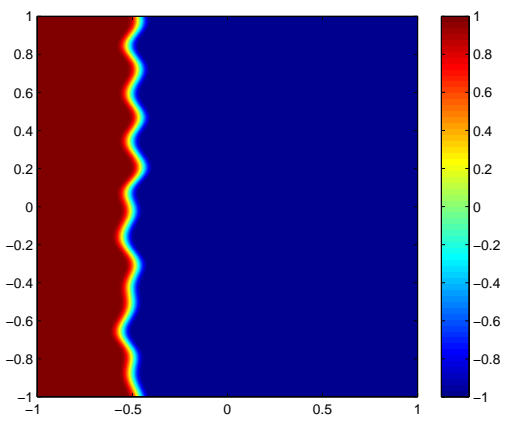

(a) $t=0$

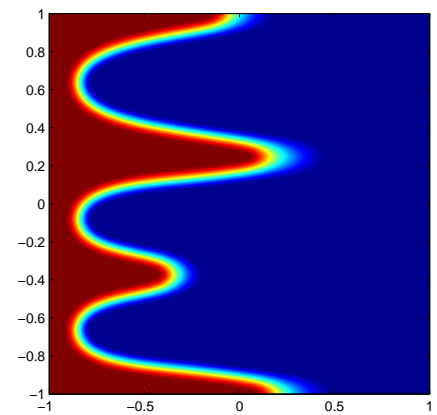

(c) $t=4 * 10^{-4}$

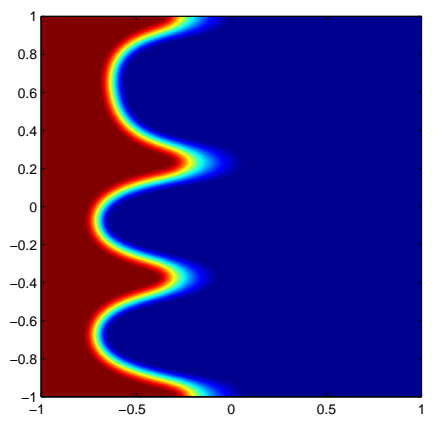

(b) $t=2 * 10^{-4}$

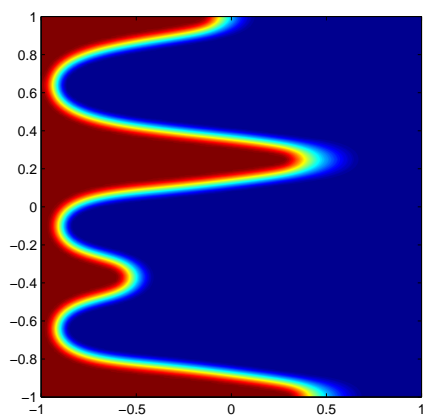

(d) $t=6 * 10^{-4}$

Figure 2: Morphology evolution of phase concentration $\left(\right.$ on $\left.\Omega_{f}\right)$ in the system $(3.1 \mathrm{a}-\mathrm{c}$ ) for $d=2$.

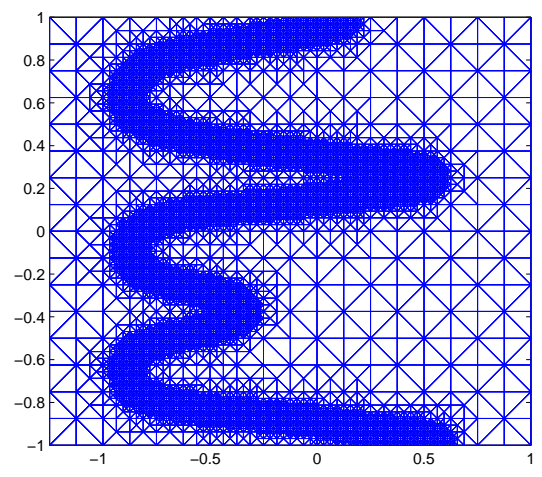

Figure 3: The underlying mesh on $\Omega$, including the substrate and film regions for the solution to $(3.16 \mathrm{a}-\mathrm{c})$ at time $T=6 * 10^{-4}$. Parameters are as in Figure 2. We note that the substrate mesh is refined to ensure there are no hanging nodes on the boundary with the film mesh. 


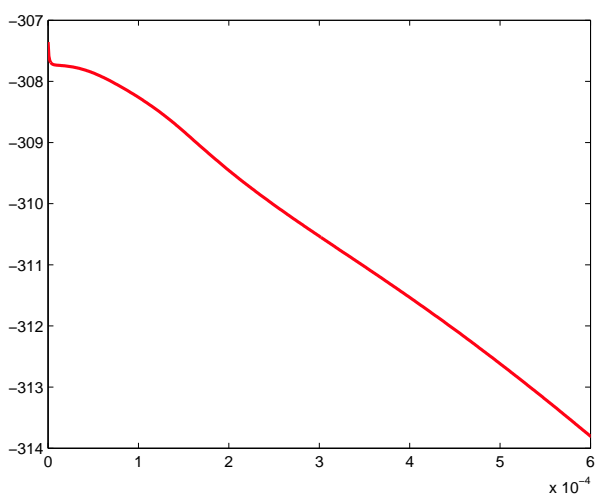

Figure 4: A plot of $\mathcal{J}\left(U^{n}, \Phi^{n}\right)$ over time for the system $(3.1 \mathrm{a}-\mathrm{c})$ for $d=2$. Parameters are as in Figure 2.

tracking the evolution of the interface.

Similarly to the two dimensional case, the evolution shows that initial perturbations in the interface coagulate together, and in this case, in an approximately uniform arrangement, see Figure 5b. This uniform arrangement is preserved as the morphology progresses and the pillar structures extend outwards into the film region, see Figures 5c and $5 \mathrm{~d}$. The plot in Figure 7 demonstrates the decreasing discrete energy for the solution of $(3.1 \mathrm{a}-\mathrm{c})$.

Acknowledgement: The authors would like to thank John W. Barrett for valuable discussions.

\section{Appendix}

In this appendix we collect some results that are used in paper. In cases where the needed results do not appear to have been derived in the literature, we also present proofs.

Let $\mathcal{G}_{0}: S_{0}^{\prime} \times L^{2}\left(\partial_{N} \Omega\right) \rightarrow S_{0}$ be defined such that

$$
\left(\underline{\nabla}\left[\mathcal{G}_{0}(z, \mu)\right], \underline{\nabla} \eta\right)=\langle z, \eta\rangle_{S_{0}}+\int_{\partial_{N} \Omega} \mu \eta \mathrm{d} s \quad \forall \eta \in S_{0},
$$

where $\langle\cdot, \cdot\rangle_{S_{0}}$ denotes the duality pairing between $S_{0}^{\prime}$ and $S_{0}$. The well-posedness of $\mathcal{G}_{0}$ follows from the Lax-Milgram theorem, a trace inequality and a Friedrich's inequality. Furthermore, we define the short-hand notation $\mathcal{G}_{0} z:=\mathcal{G}_{0}(z, 0)$, i.e. $\mathcal{G}_{0}: S_{0}^{\prime} \rightarrow S_{0}$ is such that

$$
\left(\underline{\nabla}\left(\mathcal{G}_{0} z\right), \underline{\nabla} \eta\right)=\langle z, \eta\rangle_{S_{0}} \quad \forall \eta \in S_{0} .
$$

As $\Omega$ is a convex polytope, for later use we recall the well-known result

$$
\|\eta\|_{2} \leq C(\Omega)\left[|\Delta \eta|_{0}+\left\|\frac{\partial \eta}{\partial \underline{\nu}_{\partial \Omega}}\right\|_{\frac{1}{2}, \partial_{N} \Omega}\right] \quad \forall \eta \in H^{2}(\Omega) \cap S_{0}
$$




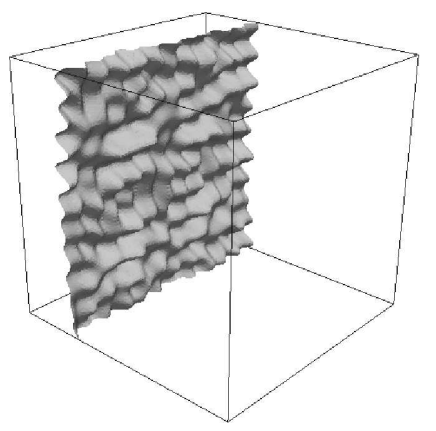

(a) $t=0$

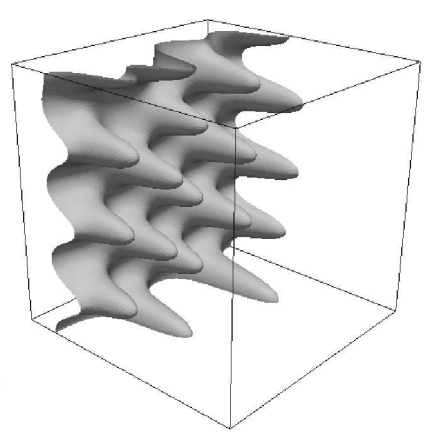

(c) $t=2 * 10^{-4}$

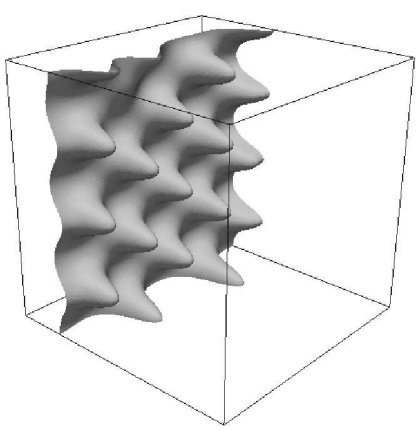

(b) $t=1 * 10^{-4}$

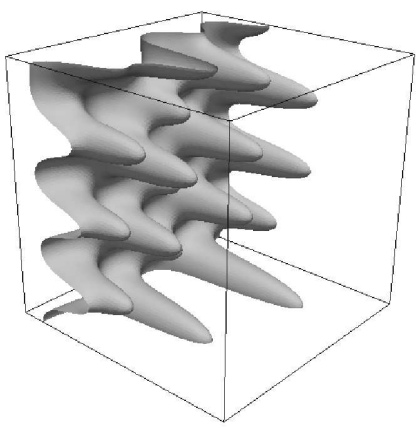

(d) $t=3 * 10^{-4}$

Figure 5: Morphology evolution of system $(3.1 \mathrm{a}-\mathrm{c})$ for $d=3$.

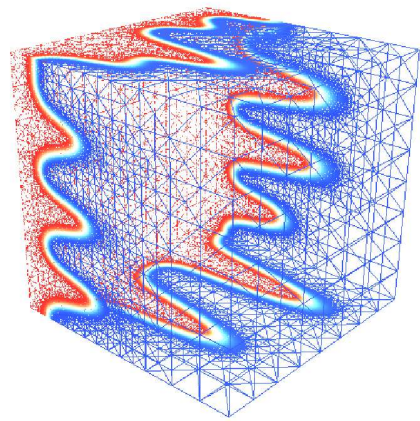

Figure 6: Mesh for $U$ on $\Omega_{f}$ at time $t=3 * 10^{-4}$ for $d=3$, for the system (3.1a-c) with parameters as in Figure 5. The mesh here is the underlying mesh for the morphology in Figure 5d. 


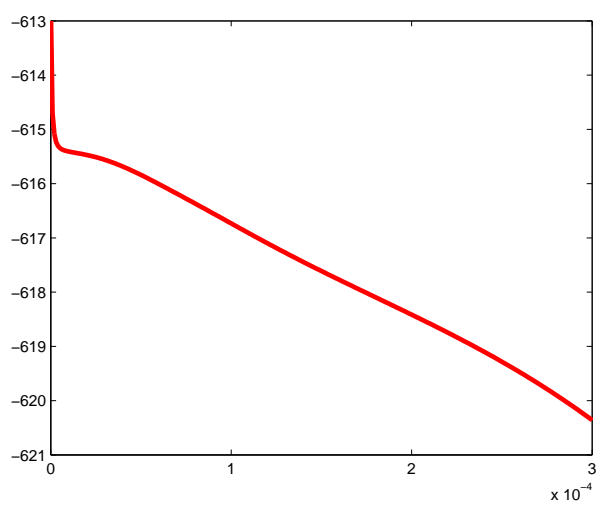

Figure 7: A plot of $\mathcal{J}\left(U^{n}, \Phi^{n}\right)$ over time for the system $(3.1 \mathrm{a}-\mathrm{c})$ for $d=3$. Parameters are as in Figure 5.

Furthermore we have the following result

$$
\|\eta\|_{2, p} \leq C(\Omega)\left[|\Delta \eta|_{0, p}+\|\eta\|_{1, p}\right] \quad \forall \eta \in W^{2, p}(\Omega) \cap S_{0} \text { with } \frac{\partial \eta}{\partial \underline{\nu}_{\partial \Omega}}=0 \text { on } \partial_{N} \Omega,
$$

for $p \in[2, \infty)$, see e.g. [18, Theorem 4.3.2.4].

Lemma A.1. Let $d=2$. Let $\mu \in H^{\frac{1}{2}}\left(\partial_{N} \Omega\right)$ with $\mu=0$ on $\overline{\partial_{D} \Omega} \cap \overline{\partial_{N} \Omega}$. Then it holds that

$$
\left\|\mathcal{G}_{0}(z, \mu)\right\|_{2} \leq C(\Omega)\left[|z|_{0}+\|\mu\|_{\frac{1}{2}, \partial_{N} \Omega}\right] \quad \forall z \in L^{2}(\Omega) .
$$

Furthermore we have, for all $p \in[2, \infty)$, that

$$
\left\|\mathcal{G}_{0} z\right\|_{2, p} \leq C(\Omega)|z|_{0, p} \quad \forall z \in L^{2}(\Omega) .
$$

Proof The proof uses several results from [19]. We present some notation regarding our domain $\Omega$, adopting the notation of [19, Figure 1, pXIII]. We define $S_{1}=\left(-L_{1}-\right.$ $\left.a,-L_{2}\right), S_{2}=\left(L_{1},-L_{2}\right), S_{3}=\left(L_{1}, L_{2}\right), S_{4}=\left(-L_{1}-a, L_{2}\right)$ and $\Gamma_{1}=\left\{\underline{x} \in \partial \Omega: x_{1}=\right.$ $\left.-L_{1}-a\right\}, \Gamma_{2}=\left\{\underline{x} \in \partial \Omega: x_{2}=-L_{2}\right\}, \Gamma_{3}=\left\{\underline{x} \in \partial \Omega: x_{1}=L_{1}\right\}$ and $\Gamma_{4}=\left\{\underline{x} \in \partial \Omega: x_{2}=\right.$ $\left.L_{2}\right\}$ so that $\cup_{j=1}^{4}\left\{S_{j}\right\}=\overline{\partial_{D} \Omega} \cap \overline{\partial_{N} \Omega}$, and $\partial_{D} \Omega=\Gamma_{1} \cup \Gamma_{3}, \partial_{N} \Omega=\Gamma_{2} \cup \Gamma_{4}$. Finally we let $\omega_{j}$ be the angles between the boundaries at the vertices $S_{j}$, that is, $\omega_{j}=\frac{\pi}{2}$ for $j=1 \rightarrow 4$. The notation is described graphically in Figure 8.

We begin with the proof of (A.6), and first consider the case $p=2$. Let $z \in L^{2}(\Omega)$. It follows from $\left[19\right.$, Theorem 2.4.3] that there exist unique $c_{j, m} \in \mathbb{R}$ such that

$$
\mathcal{G}_{0} z-\sum_{j=1}^{4} \sum_{0<\lambda_{j, m}<1} c_{j, m} \mathcal{S}_{j, m} \in H^{2}(\Omega),
$$

where $\mathcal{S}_{j, m}\left(r_{j}, \theta_{j}\right)=\eta_{j}\left(r_{j}\right) r_{j}^{\lambda_{j, m}} \phi_{j, m}\left(\theta_{j}\right)$ for $m \in \mathbb{N}, j=1 \rightarrow 4$. The $c_{j, m}$ coefficients are stress intensity factors, dependent on $z$, and $\left(r_{j}, \theta_{j}\right)$ are local polar coordinates around $S_{j}, j=1 \rightarrow 4$. We note that the $\eta_{j} \in C^{\infty}(\bar{\Omega})$ are truncation functions, and that $\eta_{j}=1$ 


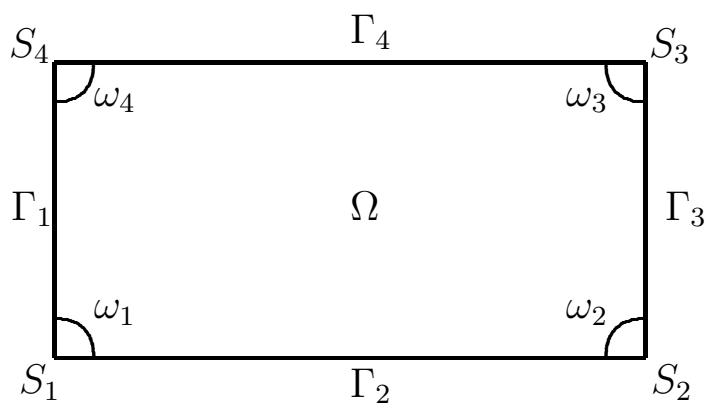

Figure 8: Diagram of the domain $\Omega$ for $d=2$, demonstrating the notation we use in the proof of Lemma A.1. We note that $\Gamma_{1} \cup \Gamma_{3}$ is the Dirichlet boundary, and $\Gamma_{2} \cup \Gamma_{4}$ is the Neumann boundary.

in a neighbourhood of the vertex $S_{j}$, but $\eta_{j}$ vanishes in a neighbourhood of all $\Gamma_{k}$ with $S_{j} \notin \overline{\Gamma_{k}}$. Moreover, $\operatorname{supp}\left(\eta_{i}\right) \cap \operatorname{supp}\left(\eta_{j}\right)=\emptyset$ if $i \neq j$.

Furthermore, $\phi_{j, m}, \lambda_{j, m}$ are the normalised eigenfunctions and eigenvalues of $\phi_{j, m}^{\prime \prime}+$ $\lambda_{j, m}^{2} \phi_{j, m}=0$ subject to the mixed boundary conditions that hold locally around $S_{j}$. We have from $[19, \mathrm{p} 50]$ that

$$
\lambda_{j, m}=\left(m-\frac{1}{2}\right) \frac{\pi}{\omega_{j}}=\left(m-\frac{1}{2}\right) 2=2 m-1 \geq 1 \quad \forall m \in \mathbb{N}, j=1 \rightarrow 4 .
$$

Therefore $\mathcal{G}_{0} z \in H^{2}(\Omega) \cap S_{0}$, integration by parts in (A.2), and (A.3) yield that

$$
\left\|\mathcal{G}_{0} z\right\|_{2} \leq C(\Omega)\left|\Delta\left(\mathcal{G}_{0} z\right)\right|_{0}=C(\Omega)|z|_{0} .
$$

To obtain the result (A.6) for $p \in(2, \infty)$, we note from $[19, \mathrm{p} 82]$ that for any $z \in L^{p}(\Omega)$

$$
\mathcal{G}_{0} z-\sum_{j=1}^{4} \sum_{0<\lambda_{j, m}<2-\frac{2}{p}} c_{j, m} \mathcal{S}_{j, m} \in W^{2, p}(\Omega),
$$

provided that $\lambda_{j, m} \neq 2-\frac{2}{p}$ for all $m \in \mathbb{N}, j=1 \rightarrow 4$. On noting that $2-\frac{2}{p} \in(1,2)$, it follows from (A.8) that this condition is satisfied, and therefore

$$
\mathcal{G}_{0} z-\sum_{j=1}^{4} c_{j, 1} \mathcal{S}_{j, 1} \in W^{2, p}(\Omega) .
$$

However, as $S_{j, 1} \in C^{\infty}(\bar{\Omega})$, recall $\lambda_{j, 1}=1$, it follows that $\mathcal{G}_{0} z \in W^{2, p}(\Omega)$. Therefore (A.4) gives us that

$$
\left\|\mathcal{G}_{0} z\right\|_{2, p} \leq C(\Omega)\left[\left|\Delta\left(\mathcal{G}_{0} z\right)\right|_{0, p}+\left\|\mathcal{G}_{0} z\right\|_{1, p}\right]=C(\Omega)\left[|z|_{0, p}+\left\|\mathcal{G}_{0} z\right\|_{1, p}\right] .
$$

Now, by Sobolev embedding theory and (A.9), we have that

$$
\left\|\mathcal{G}_{0} z\right\|_{1, p} \leq C\left\|\mathcal{G}_{0} z\right\|_{2} \leq C(\Omega)|z|_{0} \leq C(\Omega)|z|_{0, p} \quad \forall p \in(2, \infty),
$$


and therefore we have the desired result (A.6).

It follows from our assumption on $\mu$ and [19, Remark 2.4.5] that (A.7) holds, with $\mathcal{G}_{0} z$ replaced by $\mathcal{G}_{0}(z, \mu)$, i.e. $\mathcal{G}_{0}(z, \mu) \in H^{2}(\Omega)$. Then (A.3) yields that

$$
\left\|\mathcal{G}_{0}(z, \mu)\right\|_{2} \leq C(\Omega)\left[\left|\Delta \mathcal{G}_{0}(z, \mu)\right|_{0}+\left\|\frac{\partial \mathcal{G}_{0}(z, \mu)}{\partial \underline{\nu}_{\partial \Omega}}\right\|_{\frac{1}{2}, \partial_{N} \Omega}\right]=C(\Omega)\left[|z|_{0}+\|\mu\|_{\frac{1}{2}, \partial_{N} \Omega}\right],
$$

and hence (A.5) holds.

Lemma A.2. (Sobolev-Poincaré inequality) There exists a constant $C(d, r)$ such that

$$
\left(f_{D}\left|\eta-f_{D} \eta \mathrm{d} \underline{x}\right|^{r^{*}} \mathrm{~d} \underline{x}\right)^{\frac{1}{r^{*}}} \leq C(d, r) \operatorname{diam}(D)\left(f_{D}|\underline{\nabla} \eta|^{r} \mathrm{~d} \underline{x}\right)^{\frac{1}{r}},
$$

for all cuboids $D \subset \mathbb{R}^{d}$ and all $\eta \in W^{1, r}(D)$. Here $r \in[1, d)$, with $r^{*}=\frac{d r}{d-r}$.

Proof The proof of this result for $r \in(1, d)$ can be found in [16, Theorem A.2], and the proof of the general result is similar to the proof of (A.23).

Lemma A.3. Let $Q \subset \mathbb{R}^{d}$ be a cube, $g \in L_{\text {loc }}^{q}(Q)$ for $q>1$ and $g \geq 0$. Suppose that there exists $a \varsigma \in \mathbb{R}_{>0}$ and $\varrho \in L_{l o c}^{r}(Q)$ with $r>q$ and $\varrho \geq 0$ such that

$$
f_{Q_{R}\left(\underline{x}_{0}\right)} g^{q} \mathrm{~d} \underline{x} \leq \varsigma\left(f_{Q_{2 R}\left(\underline{x}_{0}\right)} g \mathrm{~d} \underline{x}\right)^{q}+f_{Q_{2 R}\left(\underline{x}_{0}\right)} \varrho^{q} \mathrm{~d} \underline{x},
$$

for each $\underline{x}_{0} \in Q$ and for all $R>0$ with $2 R<\operatorname{dist}\left(\underline{x}_{0}, \partial Q\right)$. Then $g \in L_{\text {loc }}^{s}(Q)$ for $s \in[q, q+\epsilon]$ for some $\epsilon>0$ and

$$
\left(f_{Q_{R}\left(\underline{x}_{0}\right)} g^{s} \mathrm{~d} \underline{x}\right)^{\frac{1}{s}} \leq C\left[\left(f_{Q_{2 R}\left(\underline{x}_{0}\right)} g^{q} \mathrm{~d} \underline{x}\right)^{\frac{1}{q}}+\left(f_{Q_{2 R}\left(\underline{x}_{0}\right)} \varrho^{s} \mathrm{~d} \underline{x}\right)^{\frac{1}{s}}\right],
$$

where $C$ and $\epsilon$ depend on $\varsigma q, d$ and $r$.

Proof For the proof, see [17, Proposition 5.1].

Lemma A.4. For $\underline{F} \in\left[L^{2}(\Omega)\right]^{d}$ let $f_{F} \in S_{0}$ be the unique solution of

$$
\left(\underline{\nabla} f_{F}, \underline{\nabla} \eta\right)=(\underline{F}, \underline{\nabla} \eta) \quad \forall \eta \in S_{0} .
$$

Then there exist a $\delta>0$ such that for all $p \in[2,2+\delta]$ it holds that

$$
\left|\underline{\nabla} f_{F}\right|_{0, p} \leq C(p)\left[\left|\underline{\nabla} f_{F}\right|_{0}+|\underline{F}|_{0, p}\right],
$$

if $\underline{F} \in\left[L^{p}(\Omega)\right]^{d}$. 
Proof The proof is similar to the proofs of [16, Lemma 4.1]. It is based on a Caccioppoli inequality, and a reverse Hölder inequality.

1. Interior estimate. Let $\underline{x}_{0} \in \Omega$ and $R>0$ be such that

$$
Q_{2 R}\left(\underline{x}_{0}\right):=\left\{\underline{x} \in \mathbb{R}^{d}:\left|\underline{x}-\underline{x}_{0}\right|_{\infty}<2 R\right\} .
$$

Let $\underline{x}_{0}$ and $R$ be such that $Q_{2 R}\left(\underline{x}_{0}\right) \subset \Omega$. We define a cutoff function $\zeta \in C_{0}^{\infty}\left(\mathbb{R}^{d}\right)$ with the properties

(i) $\zeta=0$ in $\mathbb{R}^{d} \backslash Q_{2 R}\left(\underline{x}_{0}\right)$,

(ii) $0<\zeta \leq 1$ in $Q_{2 R}\left(\underline{x}_{0}\right)$ and $\zeta=1$ in $Q_{R}\left(\underline{x}_{0}\right)$,

(iii) $|\underline{\nabla} \zeta| \leq \frac{2}{R}$ in $Q_{2 R}\left(\underline{x}_{0}\right)$.

We choose $\eta=\zeta^{2}\left(f_{F}-\mu\right) \in S_{0}$ in (A.10), where $\mu \in \mathbb{R}$, and obtain that

$$
\int_{\Omega} \zeta^{2}\left|\underline{\nabla} f_{F}\right|^{2} \mathrm{~d} \underline{x}=-2 \int_{\Omega} \zeta\left(f_{F}-\mu\right) \underline{\nabla} f_{F} \cdot \underline{\nabla} \zeta \mathrm{d} \underline{x}+\int_{\Omega} \underline{F}\left[2 \zeta\left(f_{F}-\mu\right) \underline{\nabla} \zeta+\zeta^{2} \underline{\nabla} f_{F}\right] \mathrm{d} \underline{x} .
$$

Applying (2.2) to (A.12) gives

$$
\int_{\Omega} \zeta^{2}\left|\underline{\nabla} f_{F}\right|^{2} \mathrm{~d} \underline{x} \leq C\left[\int_{\Omega}|\underline{\nabla} \zeta|^{2}\left(f_{F}-\mu\right)^{2} \mathrm{~d} \underline{x}+\int_{\Omega} \zeta^{2}|\underline{F}|^{2} \mathrm{~d} \underline{x}\right] .
$$

It follows from (i),(ii),(iii) and (A.13) that

$$
\int_{Q_{R}\left(\underline{x}_{0}\right)}\left|\underline{\nabla} f_{F}\right|^{2} \mathrm{~d} \underline{x} \leq C\left[\frac{1}{R^{2}} \int_{Q_{2 R}\left(\underline{x}_{0}\right)}\left(f_{F}-\mu\right)^{2} \mathrm{~d} \underline{x}+\int_{Q_{2 R}\left(\underline{x}_{0}\right)}|\underline{F}|^{2} \mathrm{~d} \underline{x}\right] .
$$

Now we choose $\mu=f_{Q_{2 R}\left(\underline{x}_{0}\right)} f_{F} \mathrm{~d} \underline{x}$ in (A.14), and apply Lemma A.2 over $Q_{2 R}\left(\underline{x}_{0}\right)$ with $r^{*}=2$, i.e. $r=\frac{2 d}{d+2}$, to obtain

$$
f_{Q_{R}\left(\underline{x}_{0}\right)}\left|\underline{\nabla} f_{F}\right|^{2} \mathrm{~d} \underline{x} \leq \widetilde{C}\left[\left(f_{Q_{2 R}\left(\underline{x}_{0}\right)}\left|\underline{\nabla} f_{F}\right|^{\frac{2 d}{d+2}} \mathrm{~d} \underline{x}\right)^{\frac{d+2}{d}}+f_{Q_{2 R}\left(\underline{x}_{0}\right)}|\underline{F}|^{2} \mathrm{~d} \underline{x}\right] .
$$

Noting (A.15) and applying Lemma A.3 with $q=\frac{d+2}{d}, g=\left|\underline{\nabla} f_{F}\right|^{\frac{2}{q}}$ and $\varrho=$ $\left(\widetilde{C}|\underline{F}|^{2}\right)^{\frac{1}{q}} \in L^{r}\left(Q_{2 R}\left(\underline{x}_{0}\right)\right)$ for some $r>q$ implies that $\left|\underline{\nabla} f_{F}\right|^{\frac{2}{q}} \in L^{s}\left(Q_{R}\left(\underline{x}_{0}\right)\right)$ for $s \in[q, q+\epsilon]$ is such that

$$
\left(f_{Q_{R}\left(\underline{x}_{0}\right)}\left|\underline{\nabla} f_{F}\right|^{\frac{2 s}{q}} \mathrm{~d} \underline{x}\right)^{\frac{1}{s}} \leq C\left[\left(f_{Q_{2 R}\left(\underline{x}_{0}\right)}\left|\underline{\nabla} f_{F}\right|^{2} \mathrm{~d} \underline{x}\right)^{\frac{1}{q}}+\left(f_{Q_{2 R}\left(\underline{x}_{0}\right)}|\underline{F}|^{\frac{2 s}{q}} \mathrm{~d} \underline{x}\right)^{\frac{1}{s}}\right] .
$$


Therefore we have that there exists a $\delta>0$ such that $\underline{\nabla} f_{F} \in L_{\mathrm{loc}}^{p}(Q)$ for $p \in[2,2+\delta]$ and

$$
\left(f_{Q_{R}\left(\underline{x}_{0}\right)}\left|\underline{\nabla} f_{F}\right|^{p} \mathrm{~d} \underline{x}\right)^{\frac{1}{p}} \leq C\left[\left(f_{Q_{2 R}\left(\underline{x}_{0}\right)}\left|\underline{\nabla} f_{F}\right|^{2} \mathrm{~d} \underline{x}\right)^{\frac{1}{2}}+\left(f_{Q_{2 R}\left(\underline{x}_{0}\right)}|\underline{F}|^{p} \mathrm{~d} \underline{x}\right)^{\frac{1}{p}}\right],
$$

where $C$ is independent of $R$. From this we obtain, on applying (2.3) that

$$
\left|\underline{\nabla} f_{F}\right|_{0, p, Q_{R}\left(\underline{x}_{0}\right)}^{p} \leq C\left[R^{d\left(1-\frac{p}{2}\right)}\left|\underline{\nabla} f_{F}\right|_{0,2, Q_{2 R}\left(\underline{x}_{0}\right)}^{p}+|\underline{F}|_{0, p, Q_{2 R}\left(\underline{x}_{0}\right)}^{p}\right] .
$$

Given an open domain $\Omega^{\prime} \subset \Omega$ with $\overline{\Omega^{\prime}} \subset \Omega$, we now apply a covering argument. Let $2 R<\operatorname{dist}\left(\overline{\Omega^{\prime}}, \partial \Omega\right)$ and choose finitely many points $\underline{x}_{i} \in \Omega^{\prime}, i=1 \rightarrow K_{R}$, such that $\overline{\Omega^{\prime}} \subset \cup_{i=1}^{K_{R}} Q_{R}\left(\underline{x}_{i}\right)$ with $Q_{R}\left(\underline{x}_{i}\right) \cap Q_{R}\left(\underline{x}_{j}\right)=\emptyset$ for $i \neq j$.

Then it follows from (A.16) and a Hölder inequality that

$$
\begin{aligned}
& \left|\underline{\nabla} f_{F}\right|_{0, p, \Omega^{\prime}}^{p} \leq \sum_{i=1}^{K_{R}}\left|\underline{\nabla} f_{F}\right|_{0, p, Q_{R}\left(\underline{x}_{i}\right)}^{p} \leq C \sum_{i=1}^{K_{R}}\left[R^{d\left(1-\frac{p}{2}\right)}\left|\underline{\nabla} f_{F}\right|_{0,2, Q_{2 R}\left(\underline{x}_{i}\right)}^{p}+|\underline{F}|_{0, p, Q_{2 R}\left(\underline{x}_{i}\right)}^{p}\right] \\
& \quad \leq C \sum_{i=1}^{K_{R}} R^{d\left(1-\frac{p}{2}\right)}\left|\underline{\nabla} f_{F}\right|_{0,2, Q_{2 R}\left(\underline{x}_{i}\right)}^{p}+C|\underline{F}|_{0, p, \Omega}^{p} \\
& \quad \leq C\left(\sum_{i=1}^{K_{R}} R^{d}\right)^{1-\frac{p}{2}}\left(\sum_{i=1}^{K_{R}}\left|\underline{\nabla} f_{F}\right|_{0,2, Q_{2 R}\left(\underline{x}_{i}\right)}^{2}\right)^{\frac{p}{2}}+C|\underline{F}|_{0, p, \Omega}^{p} \leq C\left[\left|\underline{\nabla} f_{F}\right|_{0,2, \Omega}^{p}+|\underline{F}|_{0, p, \Omega}^{p}\right],
\end{aligned}
$$

where we have noted that $\sum_{i=1}^{K_{R}} R^{d}=K_{R} R^{d} \leq|\Omega|$. On applying (2.3) once more we obtain the following interior $L^{p}$ estimate for $\underline{\nabla} f_{F}$,

$$
\left|\underline{\nabla} f_{F}\right|_{0, p, \Omega^{\prime}} \leq C\left[\left|\underline{\nabla} f_{F}\right|_{0}+|\underline{F}|_{0, p}\right],
$$

for all $\Omega^{\prime} \subset \Omega$ with $\overline{\Omega^{\prime}} \subset \Omega$.

2. Global $L^{p}$ estimate. It remains to extend this estimate to the boundary. First let $\underline{x}_{0} \in \partial_{N} \Omega$ and $R>0$ be such that $Q_{2 R}\left(\underline{x}_{0}\right) \cap \partial_{D} \Omega=\emptyset$. As before, for $\mu \in \mathbb{R}$, we choose $\eta=\zeta\left(f_{F}-\mu\right) \in S_{0}$ in $($ A.10) and obtain

$$
\int_{Q_{R}\left(\underline{x}_{0}\right) \cap \Omega}\left|\underline{\nabla} f_{F}\right|^{2} \mathrm{~d} \underline{x} \leq C\left[\frac{1}{R^{2}} \int_{Q_{2 R}\left(\underline{x}_{0}\right) \cap \Omega}\left|f_{F}-\mu\right|^{2} \mathrm{~d} \underline{x}+\int_{Q_{2 R}\left(\underline{x}_{0}\right) \cap \Omega}|\underline{F}|^{2} \mathrm{~d} \underline{x}\right] .
$$

Letting $\mu=f_{Q_{2 R}\left(\underline{x}_{0}\right) \cap \Omega} f_{F} \mathrm{~d} \underline{x}$, and recalling Lemma A.2 we hence have that

$$
f_{Q_{R}\left(\underline{x}_{0}\right) \cap \Omega}\left|\underline{\nabla} f_{F}\right|^{2} \mathrm{~d} \underline{x} \leq C\left[\left(f_{Q_{2 R}\left(\underline{x}_{0}\right) \cap \Omega}\left|\underline{\nabla} f_{F}\right|^{\frac{2 d}{d+2}} \mathrm{~d} \underline{x}\right)^{\frac{d+2}{d}}+f_{Q_{2 R}\left(\underline{x}_{0}\right) \cap \Omega}|\underline{F}|^{2} \mathrm{~d} \underline{x}\right] .
$$


Extending $\underline{\nabla} f_{F}$ and $\underline{F}$ to zero outside $\Omega$ means we can apply Lemma A.3 to these extensions on $Q_{2 R}\left(\underline{x}_{0}\right)$ to obtain, similarly to (A.16), that

$$
\left|\underline{\nabla} f_{F}\right|_{0, p, Q_{R}\left(\underline{x}_{0}\right) \cap \Omega}^{p} \leq C\left[R^{d\left(1-\frac{p}{2}\right)}\left|\underline{\nabla} f_{F}\right|_{0,2, Q_{2 R}\left(\underline{x}_{0}\right) \cap \Omega}^{p}+|\underline{F}|_{0, p, Q_{2 R}\left(\underline{x}_{0}\right) \cap \Omega}^{p}\right] .
$$

Applying a covering argument similarly to (A.17) and combining the obtained bound with the interior estimate (A.18), we have that

$$
\left|\underline{\nabla} f_{F}\right|_{0, p, \Omega^{\prime}} \leq C\left[\left|\underline{\nabla} f_{F}\right|_{0}+|\underline{F}|_{0, p}\right]
$$

for all $\Omega^{\prime} \subset \Omega$ such that $\overline{\Omega^{\prime}} \cap \partial_{D} \Omega=\emptyset$.

Next, let $\underline{x}_{0} \in \partial_{D} \Omega$. Choosing $\eta=\zeta^{2} f_{F} \in S_{0}$ in (A.10) yields that

$$
\int_{Q_{R}\left(\underline{x}_{0}\right) \cap \Omega}\left|\underline{\nabla} f_{F}\right|^{2} \mathrm{~d} \underline{x} \leq C\left[\frac{1}{R^{2}} \int_{Q_{2 R}\left(\underline{x}_{0}\right) \cap \Omega}\left|f_{F}\right|^{2} \mathrm{~d} \underline{x}+\int_{Q_{2 R}\left(\underline{x}_{0}\right) \cap \Omega}|\underline{F}|^{2} \mathrm{~d} \underline{x}\right] .
$$

We assume that, similarly to Lemma A.2, there exists a constant $C(d, r)>0$ such that

$$
\left(f_{Q_{2 R}\left(\underline{x}_{0}\right) \cap \Omega}|\eta|^{r^{*}} \mathrm{~d} \underline{x}\right)^{\frac{1}{r^{*}}} \leq C(d, r) R\left(\left.f_{Q_{2 R}\left(\underline{x}_{0}\right) \cap \Omega} \underline{\mid \nabla} \eta\right|^{r} \mathrm{~d} \underline{x}\right)^{\frac{1}{r}},
$$

for all $\eta \in W^{1, r}\left(Q_{2 R}\left(\underline{x}_{0}\right) \cap \Omega\right)$ with $\eta=0$ on $Q_{2 R}\left(\underline{x}_{0}\right) \cap \partial_{D} \Omega$. Here $r \in[1, d)$ and $r^{*}=\frac{d r}{d-r}$.

Then it follows from (A.22) and (A.23) that (A.19) holds for $\underline{x}_{0} \in \partial_{D} \Omega$ as defined here. Similarly to (A.20) on extending $\underline{\nabla} f_{F}$ and $\underline{F}$ to $Q_{2 R}\left(\underline{x}_{0}\right)$, Lemma A.3 then yields that (A.21) holds for all $\Omega^{\prime} \subset \Omega$ with $\overline{\Omega^{\prime}} \cap \partial_{N} \Omega=\emptyset$. Combining this with (A.21) then gives the desired result (A.11).

Proof of (A.23). Let $G:=\left\{\underline{\widehat{x}} \in \mathbb{R}^{d}:|\underline{\widehat{x}}|_{\infty}<1, \widehat{x}_{1}>0\right\}$ be a half of the unit cube in $\mathbb{R}^{d}$, with $\partial_{D} G:=\left\{\underline{\widehat{x}} \in \partial G: \widehat{x}_{1}=0\right\}$. Then (2.1) and a Friedrich's inequality yield immediately that

$$
|\widehat{\eta}|_{0, r^{*}, G} \leq C(d, r)\|\widehat{\eta}\|_{1, r, G} \leq C(d, r)|\widehat{\eta}|_{1, r, G},
$$

for all $\widehat{\eta} \in W^{1, r}(G)$ with $\widehat{\eta}=0$ on $\partial_{D} G$. The change of variables $\underline{x}=R \underline{\widehat{x}}$ and $\eta(\underline{x})=$ $\widehat{\eta}(\underline{\widehat{x}})=\widehat{\eta}\left(\frac{1}{R} \underline{x}\right)$ together with a translation, and a rotation if $\underline{x}_{0} \in \partial_{D}^{+} \bar{\Omega}$, then yields that

$$
\left(R^{-d} \int_{Q_{2 R}\left(\underline{x}_{0}\right) \cap \Omega}|\eta|^{r^{*}} \mathrm{~d} \underline{x}\right)^{\frac{1}{r^{*}}} \leq C(d, r) R\left(R^{-d} \int_{Q_{2 R}\left(\underline{x}_{0}\right) \cap \Omega}|\underline{\nabla} \eta|^{r} \mathrm{~d} \underline{x}\right)^{\frac{1}{r}},
$$

for all $\eta \in W^{1, r}\left(Q_{2 R}\left(\underline{x}_{0}\right) \cap \Omega\right)$ with $\eta=0$ on $Q_{2 R}\left(\underline{x}_{0}\right) \cap \partial_{D} \Omega$. Hence (A.23) holds.

\section{References}

[1] R. A. Adams And J. Fournier, Cone conditions and properties of Sobolev spaces, J. Math. Anal. Appl., 61 (1977), pp. 713-734. 
[2] H. W. Alt, Lineare Funktionalanalysis, Springer-Verlag, Berlin, 1999.

[3] J. W. Barrett, J. F. Blowey, And H. Garcke, Finite element approximation of the Cahn-Hilliard equation with degenerate mobility, SIAM J. Numer. Anal., 37 (1999), pp. 286-318.

[4] J. W. Barrett, H. Garcke, And R. Nürnberg, Finite element approxmation of a phase field model for surface diffusion of voids in a stressed solid, Math. Comp., 75 (2006), pp. 7-41.

[5] — A parametric finite element method for fourth order geometric evolution equations, J. Comput. Phys., 222 (2007), pp. 441-462.

[6] —, Parametric approximation of surface clusters driven by isotropic and anisotropic surface energies, Interfaces Free Bound., 12 (2010), pp. 187-234.

[7] J. W. Barrett AND R. NürnBerg, Finite element approximation of a Stefan problem with degenerate Joule heating, M2AN Math. Model. Numer. Anal., 38 (2004), pp. 633-652.

[8] J. Bergh AND J. LÖFSTRÖM, Interpolation Spaces, an Introduction, SpringerVerlag, Berlin, 1976.

[9] J. F. Blowey And C. M. Elliott, The Cahn-Hilliard gradient theory for phase separation with non-smooth free energy. Part I: Mathematical analysis, European J. Appl. Math., 2 (1991), pp. 233-280.

[10] — , The Cahn-Hilliard gradient theory for phase separation with non-smooth free energy. Part II: Numerical analysis, European J. Appl. Math., 3 (1992), pp. 147-179.

[11] _- Curvature dependent phase boundary motion and parabolic double obstacle problems, in Degenerate diffusions (Minneapolis, MN, 1991), vol. 47 of IMA Vol. Math. Appl., Springer, New York, 1993, pp. 19-60.

[12] S. C. Brenner And L. R. Scott, The Mathematical Theory of Finite Element Methods (second edition), Springer-Verlag, New York, 2002.

[13] G. A. Buxton And N. Clarke, Predicting structure and property relations in polymeric photovoltaic devices, Phys. Rev. B, 74 (2006), p. 085207.

[14] J. W. CAhn And J. E. Hilliard, Free energy of a non-uniform system. I. Interfacial free energy, J. Chem. Phys., 28 (1958), pp. 258-267.

[15] P. G. Ciarlet, Numerical Linear Algebra and Optimisation, Cambridge University Press, Cambridge, 1988.

[16] H. Garcke, On a Cahn-Hilliard model for phase separation with elastic misfit, Ann. Inst. H. Poincaré Anal. Non Linéaire, 22 (2005), pp. 165-185. 
[17] M. Giaquinta And G. ModicA, Regularity results for some classes of higher order nonlinear elliptic systems, J. Reine Angew. Math., 311-312 (1979), pp. 145-169.

[18] P. Grisvard, Elliptic Problems in Nonsmooth Domains, vol. 24 of Monographs and Studies in Mathematics, Pitman (Advanced Publishing Program), Boston, MA, 1985 .

[19] P. Grisvard, Singularities in Boundary Value Problems, Springer-Verlag, New York, 1992.

[20] J. J. M. Halls, C. A. Walsh, N. C. Greenham, E. A. Marseglia, R. H. Friend, S. C. Moratti, And A. B. Holmes, Efficient photodiodes from interpenetrating polymer networks, Nature, 376 (1995), pp. 498-500.

[21] D. KIm AND W. Lu, Interface instability and nanostructure patterning, Comp. Mat. Sci., 38 (2006), pp. 418-425.

[22] W. LU AND D. KIm, Thin-film structures induced by electrostatic field and substrate kinetic constraint, App. Phys. Lett., 88 (2006), p. 153116.

[23] M. PAOlini AND C. Verdi, Asymptotic and numerical analyses of the mean curvature flow with a space-dependent relaxation parameter, Asymptotic Anal., 5 (1992), pp. 553-574.

[24] R. RAnnacher And R. Scott, Some optimal error estimates for piecewise linear finite element approximations, Math. Comp., 38 (1982), pp. 437-445.

[25] M. Renardy And R. C. Rogers, An Introduction to Partial Differential Equations, Springer-Verlag, New York, 1992.

[26] R. Rossi And G. Savaré, Gradient flows of non convex functionals in Hilbert spaces and applications, ESAIM Control Optim. Calc. Var., 12 (2006), pp. 564-614.

[27] A. Schmidt And K. G. Siebert, Design of Adaptive Finite Element Software: The Finite Element Toolbox ALBERTA, vol. 42 of Lecture Notes in Computational Science and Engineering, Springer-Verlag, Berlin, 2005.

[28] C. TAng, Two-layer organic photovoltaic cell, App. Phys. Lett., 48 (1986), p. 183.

[29] E. J. W. TuCKer, Finite Element Approximations of a Phase Field Model, based on the Cahn-Hilliard Equation in the Presence of an Electric Field and Kinetics, PhD thesis, Imperial College London, London, UK, 2013. 